\title{
The structure of disks around Herbig Ae/Be stars as traced by $\mathrm{CO}$ ro-vibrational emission $\star, \star \star$
}

\author{
G. van der Plas ${ }^{1,2,3}$, M. E. van den Ancker $^{3}$, L. B. F. M. Waters ${ }^{2,4}$, and C. Dominik ${ }^{2,5}$ \\ 1 Departamento de Astronomía, Universidad de Chile, Casilla 36-D Santiago, Chile \\ e-mail: info@gerritvanderplas.com \\ 2 Astronomical Institute Anton Pannekoek, University of Amsterdam, PO box 94249, 1090 GE Amsterdam, The Netherlands \\ 3 European Southern Observatory, Karl-Schwarzschild-Str.2, 85748 Garching bei München, Germany \\ ${ }^{4}$ SRON Netherlands Institute for Space Research, Sorbonnelaan 2, 3584 CA Utrecht, The Netherlands \\ 5 Institute for Astrophysics, Radbout University, Heyendaalseweg 135, 6525 AJ Nijmegen, The Netherlands
}

Received 25 September 2014 / Accepted 25 November 2014

\begin{abstract}
Aims. We study the emission and absorption of CO ro-vibrational lines in the spectra of intermediate mass pre-main-sequence stars with the aim to determine both the spatial distribution of the $\mathrm{CO}$ gas and its physical properties. We also aim to correlate $\mathrm{CO}$ emission properties with disk geometry.

Methods. Using high-resolution spectra containing fundamental and first overtone CO ro-vibrational emission, observed with CRIRES on the VLT, we probe the physical properties of the circumstellar gas by studying its kinematics and excitation conditions.

Results. We detect and spectrally resolve CO fundamental ro-vibrational emission in 12 of the 13 stars observed, and in two cases in absorption.

Conclusions. Keeping in mind that we studied a limited sample, we find that the physical properties and spatial distribution of the $\mathrm{CO}$ gas correlate with disk geometry. Flaring disks show highly excited $\mathrm{CO}$ fundamental emission up to $v_{\mathrm{u}}=5$, while self-shadowed disks show $\mathrm{CO}$ emission that is not as highly excited. Rotational temperatures range between $250-2000 \mathrm{~K}$. The ${ }^{13} \mathrm{CO}$ rotational temperatures are lower than those of ${ }^{12} \mathrm{CO}$. The vibrational temperatures in self-shadowed disks are similar to or slightly below the rotational temperatures, suggesting that thermal excitation or IR pumping is important in these lines. In flaring disks the vibrational temperatures reach as high as $6000 \mathrm{~K}$, suggesting fluorescent pumping. Using a simple kinematic model we show that the CO inner radius of the emitting region is $\approx 10$ au for flaring disks and $\leq 1$ au for self-shadowed disks. Comparison with hot dust and other gas tracers shows that $\mathrm{CO}$ emission from the disks around Herbig Ae/Be stars, in contrast to $\mathrm{T}$ Tauri stars, does not necessarily trace the circumstellar disk up to, or inside the dust sublimation radius, $R_{\text {subl }}$. Rather, the onset of the $\mathrm{CO}$ emission starts from $\approx R_{\text {subl }}$ for self-shadowed disks, to tens of $R_{\text {subl }}$ for flaring disks. It has recently been postulated that group I Herbig stars may be transitional disks and have gaps. Our CO observations are qualitatively in agreement with this picture. We identify the location of the CO emission in these group I disks with the inner rim of the outer disk after such a gap, and suggest that the presence of highly vibrationally excited $\mathrm{CO}$ emission and a mismatch between the rotational and vibrational temperature may be a proxy for the presence of moderately sized disk gaps in Herbig Ae/Be disks.
\end{abstract}

Key words. protoplanetary disks

\section{Introduction}

Accretion disks around young stars are currently one of the most widely studied phenomena in astronomy. These gas- and dustrich disks form as a natural consequence of the conservation of angular momentum in a collapsing cloud core, from which the star forms. As the disk itself evolves, planets are born in the dense dusty midplane, hence they are often called protoplanetary (PP) disks. The evolution, and ultimately the method of dispersion of these passive disks, holds the key to many open questions related to planet formation (e.g. Alexander et al. 2014, and references therein).

* Based on observations collected at the European Southern Observatory, Paranal, Chile (Program IDs 079.C-0349(A) and 081.C-0833(A)).

$\star \star$ Appendices are available in electronic form at

http://www . aanda.org
Protoplanetary disks have historically been studied predominantly using dust, which presents only $1 \%$ of the total circumstellar mass but carries most of the opacity. Whereas dust emission dominates the infrared (IR) spectra of PP disks, and plays an important role in the evolution of PP disks, gas and dust do not necessarily evolve co-spatially or on similar timescales. Hence, gas observations are both complementary to, and essential for, our understanding of PP disks. With the advent of 10-m class telescopes, increasing amounts of gas tracers have become available with which to study the PP disks. Each of these tracers is sensitive to a different set of physical parameters, and we can tie these parameters to a specific radial location in the disk by using the kinematic information in the line profiles: from inside the dust sublimation radius $(\approx 0.1 \mathrm{au})$ up to far in the outer disk (100 s of au), and from the mid-plane to the disk atmosphere. For a review on the diagnostic power of gas tracers see e.g. Carmona (2010). 
Table 1. Astrophysical parameters of the programme stars.

\begin{tabular}{llllllllll}
\hline \hline ID & Name & Sp. type & Group & $\begin{array}{l}\log T_{\text {eff }} \\
\log [\mathrm{K}]\end{array}$ & $\begin{array}{l}\log L_{\text {bol }} \\
\log \left[L_{\odot}\right]\end{array}$ & $\begin{array}{l}M \\
{\left[M_{\odot}\right]}\end{array}$ & $\begin{array}{l}d \\
{[\mathrm{pc}]}\end{array}$ & $\begin{array}{l}i^{\star} \\
{\left[{ }^{\circ}\right]}\end{array}$ & $\begin{array}{l}\text { PA } \\
{\left[{ }^{\circ}\right]}\end{array}$ \\
\hline 1 & HD 100546 & B9Vne & I & 4.02 & 1.62 & $2.4 \pm 0.1^{o}$ & $103_{-6}^{+7, y}$ & $42 \pm 5^{d}$ & $145 \pm 5^{d}$ \\
2 & HD 97048 & A0pshe & I & 4.00 & 1.42 & $2.5 \pm 0.2^{o}$ & $180_{-20}^{+30, y}$ & $42.8_{-2.5}^{+0.8 a}$ & $175 \pm 1^{*}$ \\
3 & HD 179218 & B9e & I & 4.02 & 1.88 & $2.7 \pm 0.2^{i}$ & $240_{-40}^{+70, y}$ & $57 \pm 2^{u}$ & $23 \pm 3^{u}$ \\
4 & HD 101412 & B9.5V & II & 4.02 & 1.40 & $2.3 \pm 0.2^{i}$ & $160^{g}$ & $80 \pm 7^{u}$ & $38 \pm 5^{u}$ \\
5 & HD 141569 & B9.5e & & 3.98 & 1.10 & $2.00^{s}$ & $99_{-8}^{+9, y}$ & $51 \pm 3^{t}$ & $356 \pm 5^{t}$ \\
6 & HD 190073 & A2IVpe & II & 3.95 & 1.92 & $2.85^{l}$ & $\geq 290^{y}$ & $23_{-23}^{+15, m}$ & $10_{-10}^{+170, m}$ \\
7 & HD 98922 & B9Ve & II & 4.02 & $2.95^{\ddagger}$ & $2.2 \pm 0.8^{x}$ & $\geq 540^{\ddagger} y$ & $45^{x}$ & - \\
8 & HD 95881 & A2III/IV & II & 3.95 & $1.34^{n}$ & $2.0 \pm 0.3^{n}$ & $170 \pm 30^{n}$ & $55^{n}$ & $103^{n}$ \\
9 & R CrA & A5IIev & II & 3.86 & - & $3.5^{r}$ & $130^{b}$ & $65^{q}$ & $180^{q}$ \\
10 & HD 135344B & F8V & & 3.82 & 1.01 & $1.7 \pm 0.2^{i}$ & $140 \pm 2^{f}$ & $14 \pm 3^{e}$ & $56 \pm 2^{e}$ \\
11 & HD 150193 & A1Ve & II & 3.95 & 1.19 & $2.3 \pm 0.2^{o}$ & $150_{-30, y}^{+50}$ & $38 \pm 9^{v}$ & $358 \pm 6^{v}$ \\
12 & HD 104237 & A4Ve+sh & II & 3.92 & 1.53 & $1.96^{k}$ & $116_{-6}^{+7, y}$ & $18_{-11}^{+14, k}$ & - \\
13 & HD 142666 & A8Ve & II & 3.88 & 1.03 & $1.8 \pm 0.3^{o}$ & $145 \pm 2^{f}$ & almost edge-on $^{h}$ & - \\
\hline
\end{tabular}

Notes. The references for the values for the effective temperature of the central star $\log T_{\text {eff }}$, the observed bolometric luminosity $\log L_{\mathrm{bol}}$, and the distance $d$ can be found in Acke et al. (2005) and references therein, unless indicated otherwise. ${ }^{(\star)}$ Inclination in degrees from face-on. ${ }^{(\star)}$ Derived from the PAH $8.6 \mu \mathrm{m}$ image of Lagage et al. (2006). ${ }^{(\dagger)}$ We do not classify this source in group I/II (Sect. 2). ${ }^{(\ddagger)}$ Ambiguity about the distance exists (Blondel \& Tjin A Djie 2006).

References. ${ }^{(a)}$ Lagage et al. (2006); ${ }^{(b)}$ Marraco \& Rydgren (1981); ${ }^{(c)}$ Acke \& van den Ancker (2006); ${ }^{(d)}$ Ardila et al. (2007); ${ }^{(e)}$ Pontoppidan et al. (2008); ${ }^{(f)}$ de Zeeuw et al. (1999); ${ }^{\left({ }^{9}\right)}$ Acke \& van den Ancker (2004); ${ }^{(h)}$ Meeus et al. (1998); ${ }^{(i)}$ van der Plas et al. $(2008) ;\left({ }^{(k)}\right.$ Grady et al. (2004); ${ }^{(l)}$ Catala et al. (2007); ${ }^{(m)}$ Eisner et al. (2004); ${ }^{(n)}$ Verhoeff et al. (2010); ${ }^{(o)}$ van Boekel et al. $(2005) ;{ }^{(q)}$ Kraus et al. (2009); ${ }^{(r)}$ Forbrich et al. (2006); ${ }^{(s)}$ Merín et al. (2004); ${ }^{(t)}$ Weinberger et al. (1999); ${ }^{(u)}$ Fedele et al. (2008); ${ }^{(v)}$ Fukagawa et al. (2003); ${ }^{\left({ }^{(w)}\right.}$ Kraus et al. (2008); ${ }^{(x)}$ Blondel \& Tjin A Djie (2006); ${ }^{(y)}$ van den Ancker et al. (1998).

Carbon monoxide $(\mathrm{CO})$ is the second most abundant molecule in the universe after $\mathrm{H}_{2}$. In contrast to $\mathrm{H}_{2}$, the rovibrational transitions of $\mathrm{CO}$ are much stronger, and hence $\mathrm{CO}$ is easier to detect. Carbon monoxide is commonly seen in emission from disks and is a versatile tracer because of its many rotational and ro-vibrational transitions that are sensitive to a wide range of physical environments. The rotational $(\Delta v=0$, sub-mm CO lines (e.g. Weintraub et al. 1989; Beckwith \& Sargent 1993; Mannings \& Sargent 1997, 2000) trace the cold outer disk and disk midplane. Hot $(T>2000 \mathrm{~K})$ gas close to the star enables the ro-vibrational first overtone ( $\Delta v=2,2.3 \mu \mathrm{m}$, e.g. Scoville et al. 1983; Carr 1989; Carr et al. 1993; Najita et al. 1996) transitions to be detected, and the warm $(T \approx 1000 \mathrm{~K})$ gas in the inner disk and disk surface are excited via the ro-vibrational fundamental transitions $(\Delta v=1,4.6 \mu \mathrm{m}$, e.g. Mitchell et al. 1990; Najita et al. 2003; Blake \& Boogert 2004; Brittain et al. 2007; Salyk et al. 2009; Bast et al. 2011; Brown et al. 2013).

Many recent studies of PP disks have focused on the socalled Herbig Ae/Be (HAeBe) stars, because their disks are often more massive and more extended (and hence brighter) than the disks around their lower-mass counterparts, the T Tauri stars. In recent years, it has become clear that dust in passive disks around these HAeBe stars comes in two varieties: flaring (group I) and self-shadowed (group II) disks (Meeus et al. 2001). This classification is based on the topology of the dust in the PP disk, and the amount of small dust grains in direct view of the central star that can reprocess stellar light to IR photons, and so cause the infrared excess. Disks around HAeBe stars have a "puffed up" inner rim that casts a shadow over the outer disk. In flaring disks, the dust in the outer disk eventually rises out of this shadow, while the dust in self-shadowed disks does not. Maaskant et al. (2013) found that the absence of silicate emission in the infrared spectrum of some group I sources can only be explained by the presence of large gaps in the disks of these sources, leading these authors to postulate that group I and group II sources do not form an evolutionary sequence, but may instead be separate evolutionary pathways of the PP disk. At present it is unclear whether the gas has a similar morphology as the dust for both Meeus et al. group I and group II sources, or whether there are qualitative differences in the behavior of the gas between group I's and group II's as well.

In this paper, we use the $\mathrm{CO}$ fundamental and first overtone ro-vibrational transitions as a tool to trace the inner disk (inner tens of au) regions of thirteen PP disks surrounding young intermediate-mass stars. We present the studied sample, the observations, and the data reduction method in Sects. 2-4, and show the results in Sect. 5. In Sects. 6 and 7 we discuss the location of the emitting $\mathrm{CO}$ gas and the found correlations between disk shape and $\mathrm{CO}$ excitation conditions as a probe for the influence of disk structure on the emission properties of the molecular gas. We summarize our conclusions in Sect. 8.

\section{Sample description}

The observed sample consists of 12 HAeBe stars and one F8 star (HD 135344B), selected from Thé et al. (1994). Our targets have been selected to be homogeneous in mass of the central star and disk accretion activity (all have passive disks; $\dot{M} \lesssim$ $2 \times 10^{-7} M_{\odot} \mathrm{yr}^{-1}$ as measured from their $\mathrm{Br} \gamma$ luminosity listed in van den Ancker 2005), but to be heterogeneous in disk structure (as determined by the spatial distribution of the dust in the disk: four flaring disks and nine self-shadowed disks). The stellar parameters of the program stars are listed in Table 1.

Because the group I/II classification of the disk structure is based on the shape of the mid-IR emission, it is sensitive to the dust-emission from the circumstellar disks. Hence the presence of a disk hole or, to a lesser extent, a disk gap, may influence this classification. HD 141569 has a large inner dust hole extending up to $30 \mathrm{au}$ (e.g. Marsh et al. 2002), and therefore we do not classify it as a group I/II source. HD 97048, HD 100546 and HD 135344B are known to have disk gaps. The gap in the disk around HD 97048 extends out to $\approx 34$ au (Maaskant et al. 2013) 
and in HD 100546 extends between a few au and $\approx 13$ au (Grady et al. 2005; Benisty et al. 2010). Because both disks show ample signs of a flaring disk structure, we maintain the group I classification. Based on its SED, the disk around HD 135344B is also flaring. However, because of the large $(\approx 45 \mathrm{au}$; Brown et al. 2007) gap in the dust disk, and because of its late spectral type (F8), we do not classify it as a group I/II source.

\section{Observations and data reduction}

Observations: high-spectral-resolution $(R \approx 94000$, determined from telluric lines) spectra were taken with the VLT Cryogenic high-Resolution InfraRed Echelle Spectrograph (CRIRES ${ }^{1}$, Käufl et al. 2004) between June 14th and June 16th 2007 and July 2nd and July 5th 2008. Adaptive Optics were used to optimize the signal-to-noise ratio and spatial resolution of the observations. Our observations contain the wavelength ranges between 2.011-2.138 and 2.199-2.450 $\mu \mathrm{m}$ (covering the CO first overtone ro-vibrational emission), and between 4.588-4.809 and 4.905-5.094 $\mu \mathrm{m}$ (covering many fundamental ro-vibrational transitions of the $\mathrm{CO}$ molecule). The observations were made with a slit width of $0.2^{\prime \prime}$, and during the observations the slit was allowed to rotate to keep alignment with the parallactic angle.

Source acquisition and confidence of centering: during our observations the slit was allowed to rotate to keep aligned with the parallactic angle, which can smear out the signal of those sources with spatially resolved continuum and/or $\mathrm{CO}$ emission. The time on target varies between targets, but is on average $\pm 1 \mathrm{~h}$ per target, and \pm 10 min per spectral window. This translates into an average rotation of the slit parallactic angle of $15^{\circ}$ (with a maximum of $29.5^{\circ}$ for HD 135344B) per target, or a couple of degrees per wavelength setting. Compared to the PSF (0.193") this is negligible within each spectral window.

Centering of the slit on our objects was performed on the photo-center of the $K$ band flux of our objects. Some of our targets have disks with a considerable contribution to the $K$ band flux, but that excess emission comes from distances very close (within $\approx 0.5 \mathrm{au}$ ) to the star and has little influence on the photo center of the $K$ band emission. For example, $\geq 95 \%$ of the $K$ band excess of HD 100546 (total excess: $1.24 \mathrm{mag}$ ) comes from the inner disk rim at 0.25 au of the central star, while just a small percent comes from photons scattered off the inner wall of the outer disk at $\approx 13$ au (cf. Fig. 8 of Mulders et al. 2013 or Fig. 4 of Tatulli et al. 2011). An offset between the stellar position of HD 100546 and the center of the cavity of $\approx 5$ au (1/4 slit width) to the NW of the center of the cavity has been reported by Grady et al. (2005). Because the CO emission extends up to at least 35 au (i.e. beyond the slit, cf. paragraph 5.5), this introduces asymmetries in both the line spatial and spectral profile. For a complete discussion of the influence of a slit offset on spatially resolved line profiles we refer to Hein Bertelsen et al. (2014).

Data reduction: the data were reduced using the CRIRES pipeline $\mathrm{V} 1.7 .0^{2}$, which performs wavelength calibration, background subtraction and flatfield correction. For the targets without spatially resolved $\mathrm{CO}$ emission, we use the "optimal" extraction method, which uses a weighting function derived from a fit to the spatial profile. Because this method does not correctly account for extended emission, we use the "rectangular" extraction method for the spectra of the spatially resolved disks around HD 97048, HD 100546, and HD 141569.

\footnotetext{
1 http://www.eso.org/sci/facilities/paranal/ instruments/crires/

2 www.eso.org/sci/data-processing/software/pipelines/ index.html
}

To correct for telluric absorption we observed a telluric standard star directly after each science observation. Each standard was chosen to be as close as possible on the sky so that their spectra are affected by similar atmospheric conditions, and compared to an appropriate Kurucz (1991) stellar atmosphere model to determine the instrumental response. The optical depth of the telluric lines of the standard was scaled to that of the science target, and the two were ratioed using a manually determined sub-pixel wavelength shift to reduce telluric residuals (i.e. spikes due to minor wavelength mismatches). Some telluric absorption lines are fully saturated, causing problems with the division of the spectra. We inspect all resulting spectra visually for residuals of an (imperfect) telluric correction, and make a conservative estimate for the cut-off value of areas of low transmittance on a per-spectrum basis. In practice this means that we ignore all areas below a transmittance of $\approx 15 \%$ in our analysis. In the following data reduction, we will disregard these regions.

CO lines in our spectra were identified using the line list of Chandra et al. (1996). We show typical spectra for all sources with the $\mathrm{CO}$ centered on its rest wavelength in Figs. 1 and 2. The CO overtone spectra are flux calibrated using the continuum fluxes for our sources as published in the 2MASS catalog (Skrutskie et al. 2006). The CO fundamental spectra are flux calibrated with the $4.77 \mu \mathrm{m}$ flux, as determined with a spline interpolation to the $M$ band data points, from e.g. Kilkenny et al. (1985); Hillenbrand et al. (1992); de Winter et al. (2001) (see Table B.1). We fit a 2nd degree polynomial to either side of each unblended $\mathrm{CO}$ line to determine the continuum baseline. We define the FWHM as the width of the line half way between the continuum and the line maximum, and obtain the line flux by integrating the line where it is more than $1 \sigma$ above the continuum. We note that the $0.2^{\prime \prime}$ slit of CRIRES may obscure parts of the emitting region of the spatially resolved disks. $\mathrm{CO}$ emission, extended out to many tens of au has been detected around HD 97048 (van der Plas et al. 2009), HD 100546 (van der Plas et al. 2009; Brittain et al. 2009; Goto et al. 2012; Hein Bertelsen et al. 2014), and HD 141569 (Goto et al. 2006).

\section{Method}

When in local thermodynamic equilibrium (LTE), the strength and ratio of all rotational transitions within and between vibrational bands dictates an unique temperature of the gas via the Boltzmann equation. We can estimate the total amount of emitting $\mathrm{CO}$ gas $\left(N_{\text {tot }}\right)$ and its rotational temperature $\left(T_{\text {rot }}\right)$ using this Boltzmann equation and the measured line fluxes

$$
\frac{F_{i j}}{g_{i} v_{i j} A_{i j}}=\frac{1}{4 \pi d^{2}} \frac{h B N_{\text {tot }}}{T_{\text {rot }}} \mathrm{e}^{E_{i} / k T_{\text {rot }}}
$$

where $F_{i j}$ is the line flux, $v_{i j}$ the frequency of the transition, $A_{i j}$ the Einstein A-coefficient, $g_{i}$ the degeneracy of the upper level, $d$ the distance to the source, $B$ the rotational constant, and $E_{i}$ the energy of the transition.

We present our data in a so-called "Boltzmann plot" in Fig. 3. Inspection of Fig. 3 shows that some rotational transitions, most notably those of the ${ }^{12} \mathrm{CO} v_{\mathrm{u}}=1$ vibrational bands, deviate from linearity and curve upwards for lower energies. This behavior is observed frequently in $\mathrm{CO}$ emission coming from disks (e.g. Najita et al. 2003; Blake \& Boogert 2004; Brittain et al. 2007; Salyk et al. 2009; Hein Bertelsen et al. 2014), and is commonly explained by either a radial gradient in the $\mathrm{CO}$ temperature or by optically thick gas. Fitting our data with a two-temperature CO gas model yields acceptable fits, but requires for the cold 


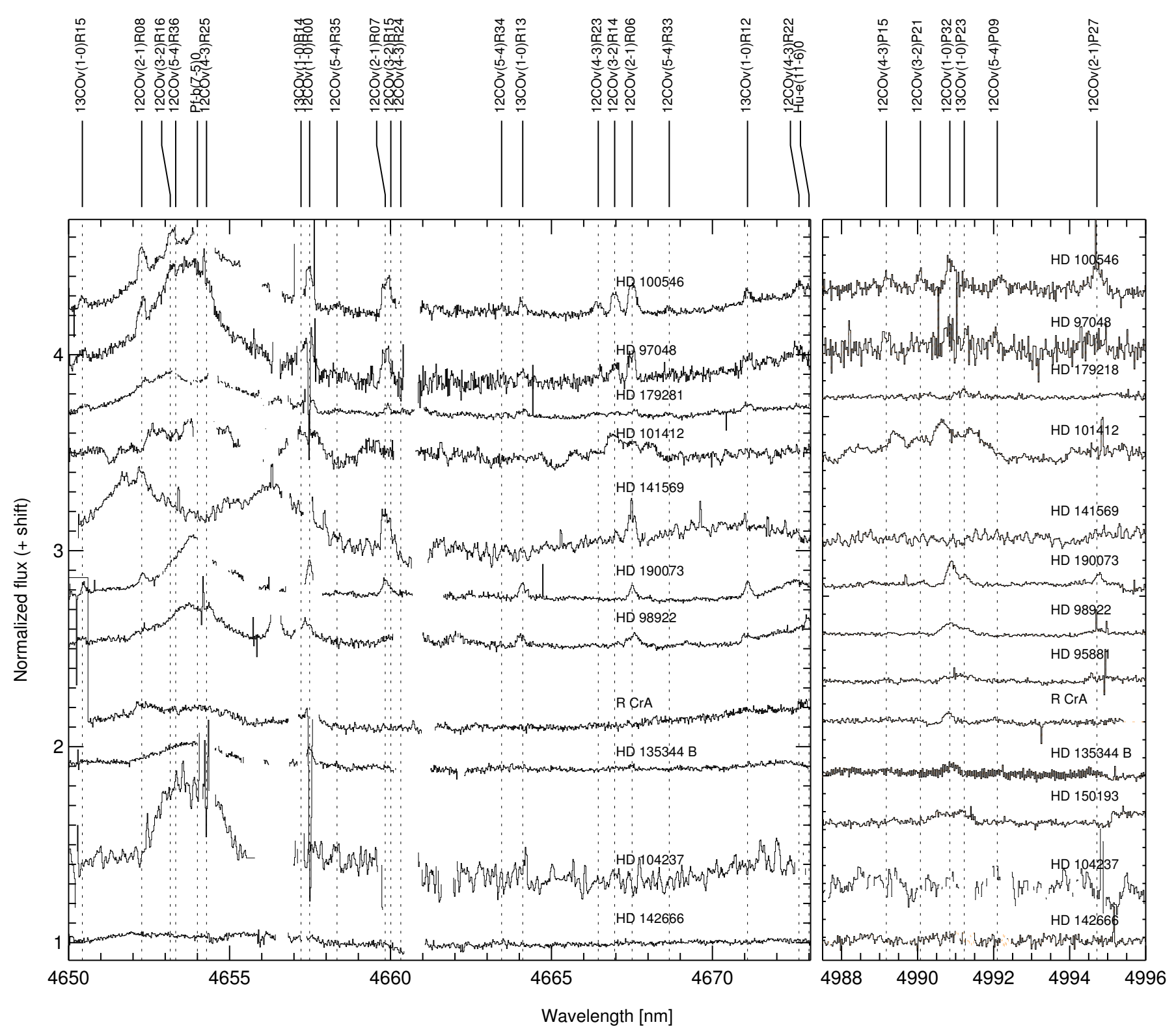

Fig. 1. Example of CRIRES spectra of our targets in the between 4650-4673 nm and 4988-4996 nm. The continuum in all spectra is normalized to 1 and subsequently vertically shifted for clarity. Regions with low transmittance have been omitted from the spectra, and the spectra of HD 95881 and HD 150193 have been omitted completely from the left panel because of problems with the telluric correction in this wavelength range.

component both unrealistically low temperatures $(\approx 65 \mathrm{~K})$, and a CO column that is over 17 orders of magnitude larger that the amount of $\mathrm{CO}$ needed to explain the warmer CO. This is incompatible with the (much broader) observed line widths in disks, and we thus interpret the curvature seen in the Boltzmann plots as originating from optical depth effects. See Salyk et al. (2009) for a similar argument regarding $\mathrm{T}$ Tauri stars.

\subsection{Rotational and vibrational temperatures}

The temperature of a gas in LTE dictates the distribution of its emission over the various rotational transitions and vibrational bands. The temperatures derived via the Boltzmann equation from the fractional distribution between the different rotational transitions within each vibrational band $\left(T_{\text {rot }}\right)$, and the temperature derived from the ratio of the total population of each vibrational band $\left(T_{\mathrm{vib}}\right)$, are thus the same for gas in LTE. Deviations between these two temperatures point to alternative, non-LTE (de)excitation mechanisms. For example, sub-thermal vibrational level populations are expected in low-density environments where the higher $\mathbf{J}$ rotational transitions are depopulated relative to LTE, while super-thermal level populations can be caused by e.g. IR pumping (Scoville et al. 1980) or UV fluorescence (Krotkov et al. 1980) .

To determine the vibrational temperature we use the cumulative best fit $\mathrm{CO}$ rotational temperature and column per source to calculate the total amount of $\mathrm{CO}$ molecules needed to explain the observed line fluxes per vibrational band. We then calculate the vibrational temperature according to the relative populations of the vibrational bands following Brittain et al. (2007). One $\sigma$ errors on the vibrational temperature are determined by adopting a relative error in $N(\mathrm{CO})$ of $25 \%$ between the vibrational bands for each individual source.

\subsection{Isothermal slab model}

We interpret the curvature in the Boltzmann plots in the subsequent analysis to be due to opacity effects. We model the 
G. van der Plas et al.: CO ro-vibrational line survey of HAeBe disks
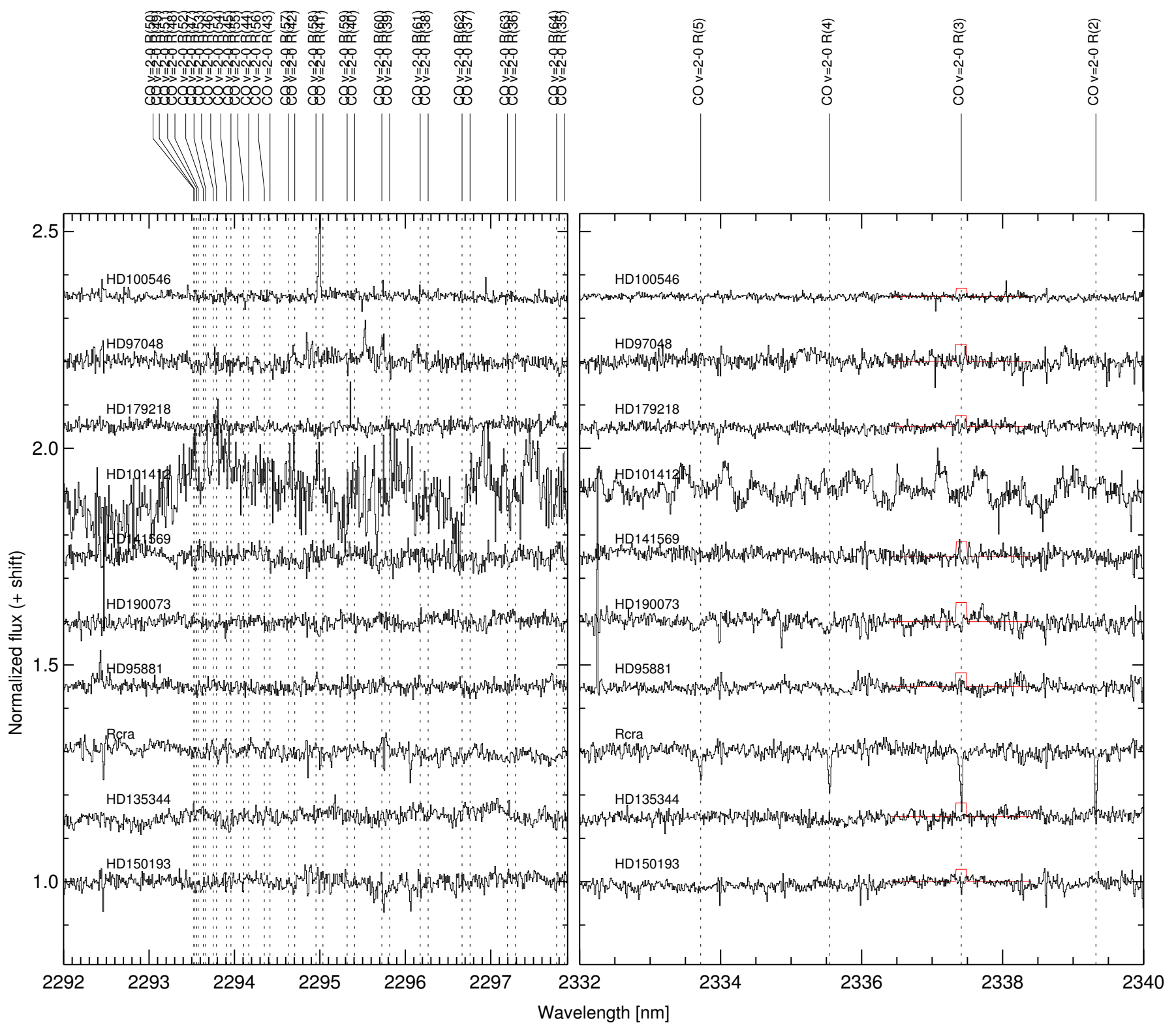

Fig. 2. Example of CRIRES spectra of our targets in the between 2292 and $2298 \mathrm{~nm}$ (the $v=2-0$ band head, left panel) and between 2332 and $2340 \mathrm{~nm}$ (the low rotational lines, right panel). The continuum in all spectra is normalized to 1 and subsequently vertically shifted for clarity. The red line in the right panel denotes the upper limit for the line fluxes noted in Sect. 5.4 and Table B.2.

emitting $\mathrm{CO}$ gas as to be originating from an isothermal slab of CO gas that is in LTE. Free parameters in this slab are the excitation temperature of the $\mathrm{CO}$ gas, $T_{\mathrm{ex}}$, the $\mathrm{CO}$ column, $N(\mathrm{CO})$, and the slab surface. A similar approach has been taken before by e.g. Goldsmith \& Langer (1999); Salyk et al. (2009); Goto et al. (2012); Thi et al. (2013); Brown et al. (2013).

We correct for optical thickness effects by defining an optical depth correction factor for each upper level column density: $\mathrm{C}_{\tau}$ (see Goldsmith \& Langer 1999, Eqs. (6), (16) and (17)). We set the line width $\Delta v$ as the FWHM value of the combined thermal and turbulent velocities. The turbulent velocity is fixed at one tenth of the sound speed (see e.g. Hughes et al. 2011; Rosenfeld et al. 2013). Within our parameter space $\Delta v$ varies between $\approx 0.5$ and $3 \mathrm{~km} \mathrm{~s}^{-1}$, and the contribution to line broadening of turbulent motions is minor. For each pair of $T_{\mathrm{ex}}$ and $N(\mathrm{CO})$ we find a best fit surface assuming the distance to the source listed in Table 1, and compare our model and observations using this best fit surface. We also use this surface calculate the vibrational temperature through the total number of emitting $\mathrm{CO}$ molecules (Fig. 4).

To find the best fit $\mathrm{CO}$ column and rotational temperature we create a grid of models and explore the parameter space between $100 \mathrm{~K} \leq T_{\text {ex }} \leq 2500 \mathrm{~K}$ and $10^{16} \mathrm{~cm}^{-2} \leq N(\mathrm{CO}) \leq$ $10^{20} \mathrm{~cm}^{-2}$ for ${ }^{12} \mathrm{CO}$ emission, and $100 \mathrm{~K} \leq T_{\mathrm{ex}} \leq 1200 \mathrm{~K}$ and $10^{17} \mathrm{~cm}^{-2} \leq N(\mathrm{CO}) \leq 10^{21} \mathrm{~cm}^{-2}$ for ${ }^{13} \mathrm{CO}$ emission, in steps of $\Delta T_{\text {ex }}=25 \mathrm{~K}$ and $\Delta^{10} \log (N(\mathrm{CO}))=0.1$. We define our best fit and $1 \sigma$ error following the reduced $\chi^{2}$ formalism. We remind the reader that the number of degrees of freedom can only be estimated for linear models (see e.g. Andrae et al. 2010), and thus that our reported $1 \sigma$ errors likely do not represent $68 \%$ confidence intervals. We use the $\chi_{r}{ }^{2}$ value in this paper to facilitate comparison with other, similar works.

Using an isothermal slab of CO gas in LTE to model the emission coming from a disk is a simplified approach. There exists a degeneracy between the line width $\Delta v$ and $N(\mathrm{CO})\left(\tau \propto \frac{1}{\Delta v}\right)$ which becomes significant when the line emission is optically 
A\&A 574, A75 (2015)
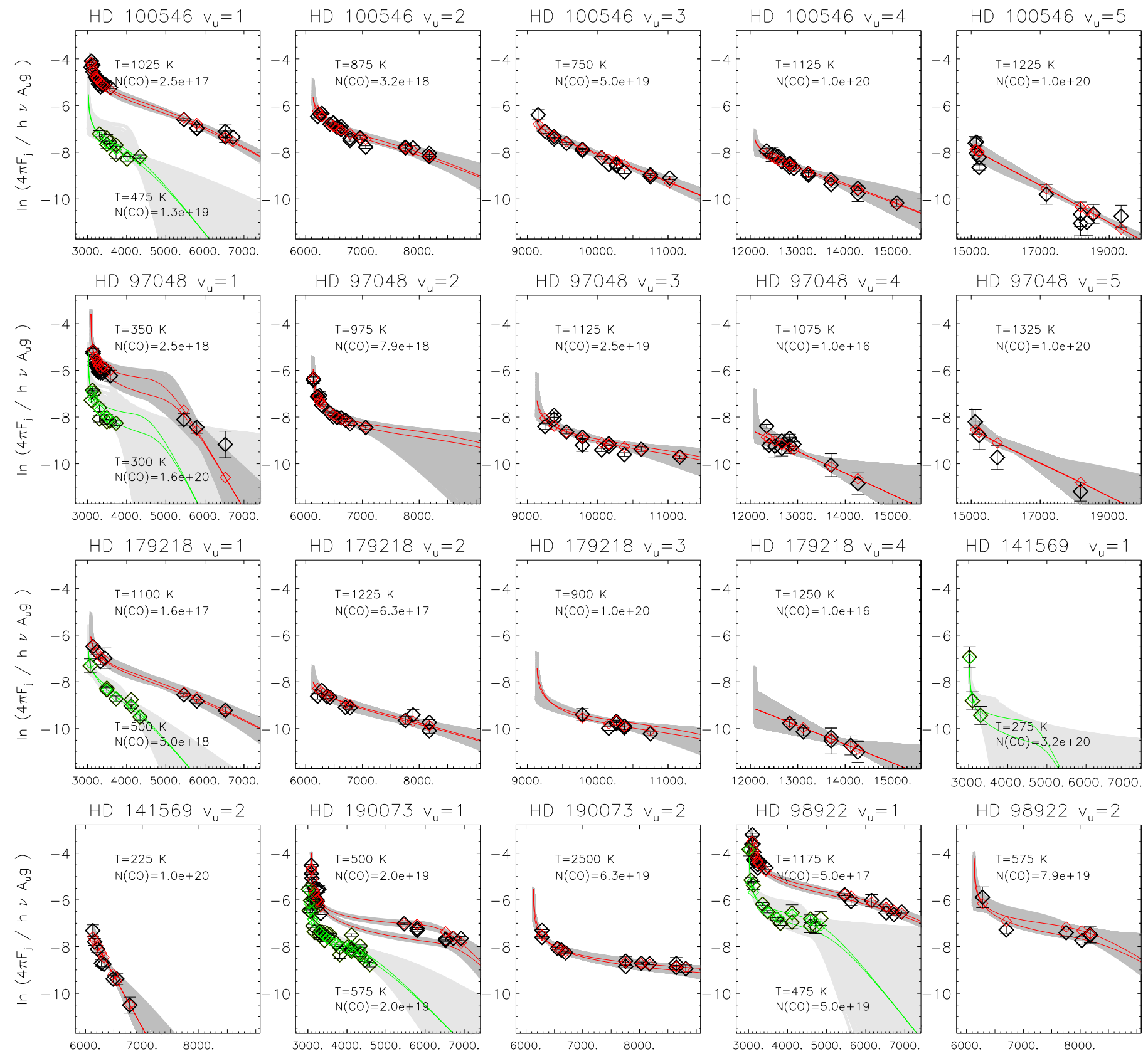

HD $98922 v_{u}=1$

3000. 4000. 5000. 6000. 7000
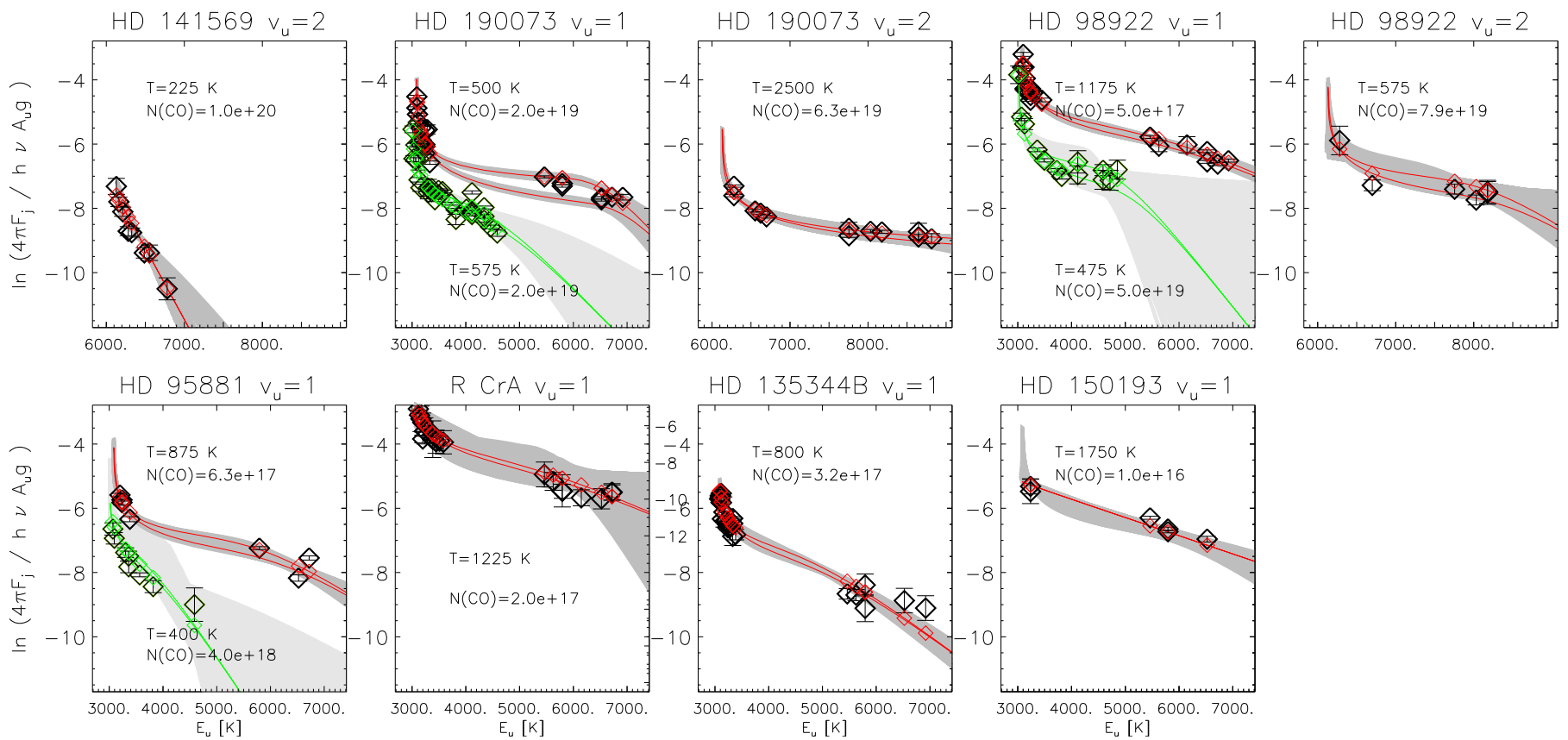

Fig. 3. Boltzmann plots for detected ${ }^{12} \mathrm{CO}$ and, if detected, ${ }^{13} \mathrm{CO}$ emission (black plot symbols). The best fit for the ${ }^{12} \mathrm{CO}$ rotational temperature $T_{\text {rot }}$ is shown by the red lines (upper line: $\mathrm{P}$ branch, lower: $\mathrm{R}$ branch), whereas the best fit $T_{\text {rot }}$ for ${ }^{13} \mathrm{CO}$ is shown by the green lines. Model predictions for the best fit $(T, N)$ are shown with the smaller open red/green diamonds. The gray area around the best fit lines show the model predictions for parameters within the $1 \sigma$ areas shown in Figs. A.1 and A.2. The Boltzmann plots for HD 101412 and HD 104237 are absent for reasons described in Sect. 5.1 
G. van der Plas et al.: CO ro-vibrational line survey of HAeBe disks

Table 2. Detected CO lines, their radial velocities with a typical error of $2 \mathrm{~km} \mathrm{~s}^{-1}$ as determined in Sect. 5.3, and the stellar radial velocities derived from photospheric absorption lines in Cols. 1-3.

\begin{tabular}{|c|c|c|c|c|c|c|c|c|c|}
\hline Name & $\begin{array}{l}v_{\mathrm{CO}} \\
\mathrm{km} \mathrm{s}^{-1}\end{array}$ & $\begin{array}{l}v_{\star} \\
\mathrm{km} \mathrm{s}^{-1}\end{array}$ & $\begin{array}{l}{ }^{12} \mathrm{CO} \\
v_{\mathrm{u}}=1\end{array}$ & $\begin{array}{l}{ }^{12} \mathrm{CO} \\
v_{\mathrm{u}}=2\end{array}$ & $\begin{array}{l}{ }^{12} \mathrm{CO} \\
v_{\mathrm{u}}=3\end{array}$ & $\begin{array}{l}{ }^{12} \mathrm{CO} \\
v_{\mathrm{u}}=4\end{array}$ & $\begin{array}{l}{ }^{12} \mathrm{CO} \\
v_{\mathrm{u}}=5\end{array}$ & $\begin{array}{l}{ }^{13} \mathrm{CO} \\
v_{\mathrm{u}}=1\end{array}$ & $\begin{array}{l}{ }^{12} \mathrm{CO} \\
v=(2-0)\end{array}$ \\
\hline \multicolumn{10}{|l|}{ Emission } \\
\hline HD 100546 & 14 & $18^{a}$ & R08-P37 & R17-P28 & $\mathrm{R} 25-\mathrm{P} 21$ & R32-P17 & R39-P09 & R18-P23 & no \\
\hline HD 97048 & 16 & $21^{a}$ & R08-P36 & R17-P07 & R26-R05 & R27-P17 & R33-P09 & R15-R04 & no \\
\hline HD 179218 & 17 & $15.4 \pm 2.3^{b}$ & R06-P36 & R14-P28 & R23-P21 & $\mathrm{R} 27-\mathrm{P} 20$ & - & $\mathrm{R} 21-\mathrm{P} 21$ & no \\
\hline HD 101412 & 16 & $16.9 \pm 0.2^{b}$ & R09-P37 & R17-P32 & R26-P26 & R35-P20 & - & R23-P29 & yes \\
\hline HD 141569 & -11 & $-12 \pm 7^{c}$ & R09-P13 ${ }^{\dagger}$ & $\mathrm{R} 14-\mathrm{P} 01^{\dagger}$ & $\ddagger$ & $\ddagger$ & $\ddagger$ & R09-R00 ${ }^{\dagger}$ & no \\
\hline HD 190073 & -1 & $0.21 \pm 1.0^{3}$ & R08-P38 & R13-P32 & - & - & - & R23-P23 & no \\
\hline HD 98922 & 3 & $0.2 \pm 2.2^{c}$ & R07-P38 & R13-P28 & - & - & - & R16-P27 & $\star$ \\
\hline HD 95881 & 18 & $36^{a}$ & R06-P37 & - & - & - & - & R16-P25 & no \\
\hline $\mathrm{R} \mathrm{CrA}$ & $-13^{\diamond}$ & $0^{d}$ & P04-P37 & - & - & - & - & - & no \\
\hline HD 135344B & 2 & $1.6 \pm 1.3^{b}$ & R08-P38 & - & - & - & - & - & no \\
\hline HD 150193 & -6 & $-4.9 \pm 3.9^{c}$ & R06-P36 & - & - & - & - & - & no \\
\hline HD 104237 & 14 & $13^{a}$ & $\mathrm{R} 08-\mathrm{P} 10^{\dagger}$ & - & - & - & - & - & $\star$ \\
\hline HD 142666 & - & $-7.0 \pm 2.7^{c}$ & - & - & - & - & - & - & $\star$ \\
\hline \multicolumn{10}{|l|}{ Absorption } \\
\hline HD 97048 & 16 & $21^{a}$ & R04-P04 & - & - & - & - & R02-P01 & no \\
\hline $\mathrm{R} \mathrm{CrA}$ & -3 & $0^{d}$ & R08-P15 & - & - & - & - & R08-P04 & R08-P08 \\
\hline
\end{tabular}

Notes. Emission lines are listed in the upper panel, and absorption lines in the lower. $\stackrel{( \pm)}{ \pm}$ Even though we do not detect these CO transitions in our spectra, they have been detected by Brittain et al. (2007) in this source. ${ }^{(\dagger)}$ Not all spectra have S/N sufficient to identify the ro-vibrational transitions, accordingly this may be a subset of all transitions. $\left.{ }^{(}\right)$The CO emission lines from R CrA most likely originate from an outflow as discussed in Sect. 5.5. ${ }^{(\bullet)}$ Radial velocity determined based on CaII lines. ${ }^{(\star)}$ No observations available.

References. References for the stellar radial velocity are: ${ }^{(a)}$ Acke et al. (2005); ${ }^{(b)}$ van der Plas et al. (2008); ${ }^{(c)}$ Alecian et al. (2013); and (d) Reipurth et al. (1996).
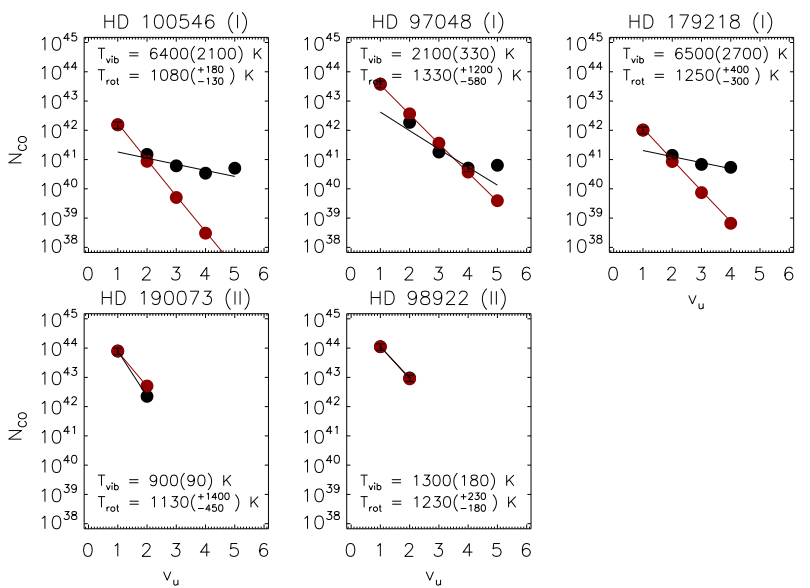

Fig. 4. Best fit vibrational (black lines) and rotational (red lines) temperatures for sources with at least $v_{\text {up }}=1,2$ detected. The target name and disk classification (group I/II) are given in each plot title. Black points show the total amount of $\mathrm{CO}$ molecules per vibrational band needed to explain the detected flux assuming the distance listed in Table 1 and an averaged CO column and $T_{\text {rot }}$. Red points show an LTE distribution calculated for the averaged $T_{\text {rot }}$. Both sets are normalized on $v_{\mathrm{u}}=1$. The errors for the rotational temperatures are $1 \sigma$ best fit contours of the summed best fit contour for all vibrational bands per source. Calculated vibrational temperature errors are $1 \sigma$ values adopting a relative error of $25 \%$ in $N(\mathrm{CO})$.

thick. It also ignores any vertical or radial temperature gradient and non LTE excitation mechanisms such as UV fluorescence and non resonant scattering of IR photons which we will discuss in Sect. 7.1. The presence of such non-thermal excitation mechanisms can be recognized by a mismatch between the rotational and vibrational temperatures derived from the Boltzmann diagrams.

We present the results of our modeling of the rotational temperatures and CO column in Table 3, and the best fit contours in Figs. A.1 and A.2. The modeling results for the vibrational temperatures are listed in Table 4 and shown in Fig. 4.

\section{Results}

\subsection{General description of the spectra}

Twelve out of thirteen surveyed HAeBe stars show spectrally resolved fundamental ${ }^{12} \mathrm{CO}$ emission, eight show fundamental ${ }^{13} \mathrm{CO}$ emission, and one shows first overtone ${ }^{12} \mathrm{CO}$ emission (Figs. 1 and 2). For the source in which we did not detect ${ }^{12} \mathrm{CO}$ emission, HD 142666, we calculate a 3 sigma upper limit on the line flux of $1.2 \times 10^{-15} \mathrm{erg} \mathrm{cm}^{-2}$ for the CO lines at $4.667 \mu \mathrm{m}$ assuming a line width of $20 \mathrm{~km} \mathrm{~s}^{-1}$ and the continuum flux reported in Sylvester et al. (1996). There is a large spread in the line widths and distribution of $\mathrm{CO}$ molecules over vibrational transitions. The $v_{\mathrm{u}}=1$ bands in all stars but HD 141569 are rotationally excited up to high $\left(J_{\text {up }}>30\right)$ transitions (Table 2). The $\frac{{ }^{12} \mathrm{CO} P 12}{{ }^{13} \mathrm{CO} R 12}$ ratio for HD 179218, and the ${ }^{12} \mathrm{CO} \mathrm{R04}$ ratio for the other targets, varies between 3 and 7.

We have been unable to characterize the spectra of two stars for which we have detected $\mathrm{CO}$ emission. The combination of broad lines $\left(F W H M \approx 80 \mathrm{~km} \mathrm{~s}^{-1}\right)$ and multiple (at least five: ${ }^{12} \mathrm{CO} v_{\mathrm{u}}=1,2,3$ and 4 , and ${ }^{13} \mathrm{CO} v_{\mathrm{u}}=1$ ) vibrational transitions in the spectrum of HD 101412 caused the emission lines to be highly blended. A combination of poor SN and telluric absorption partially overlapping with the $\mathrm{CO}$ emission made it possible to identify $\mathrm{CO}$ in the spectrum of HD 104237, but we have been unable to reliably measure line fluxes.

The CO emission in HD 97048, HD 100546, HD 141569 and $\mathrm{R}$ CrA is spatially resolved, and the $4.6 \mu \mathrm{m}$ continuum emission is resolved in HD 97048, HD 100546, and HD 179218. We discuss detected CO absorption towards HD 97048 and R CrA in Sect. 5.6, and the detected emission lines other than $\mathrm{CO}$ in Sect. 5.7. 
Table 3. Derived physical properties $\left(N_{\mathrm{CO}}, T_{\text {rot }}\right.$, emitting surface, best fit reduced $\chi^{2}$ value and FWHM) for the CO emission in our sample.

\begin{tabular}{|c|c|c|c|c|c|c|c|c|c|}
\hline Target & $v_{\mathrm{u}}$ & $\begin{array}{l}{ }^{10} \log N(\mathrm{CO}) \\
{\left[\mathrm{cm}^{-2}\right]}\end{array}$ & $\begin{array}{l}T_{\text {rot }} \\
{[\mathrm{K}]} \\
\end{array}$ & $\begin{array}{l}\text { Surface } \\
\min \\
{\left[\mathrm{au}^{2}\right]}\end{array}$ & $\begin{array}{l}\text { Surface } \\
\max \\
{\left[\mathrm{au}^{2}\right]}\end{array}$ & $\begin{array}{l}\chi_{r}^{2} \\
\min \end{array}$ & $\begin{array}{l}F W H M \\
(J<10) \\
{\left[\mathrm{km} \mathrm{s}^{-1}\right]}\end{array}$ & $\begin{array}{l}F W H M \\
(10 \leq J<20) \\
{\left[\mathrm{km} \mathrm{s}^{-1}\right]}\end{array}$ & $\begin{array}{l}F W H M \\
(J \geq 20) \\
{\left[\mathrm{km} \mathrm{s}^{-1}\right]}\end{array}$ \\
\hline HD 100546 & 1 & $17.40_{-01}^{+0.1}$ & $1025_{-100}^{+100}$ & $4.01 \mathrm{e}-01$ & $9.10 \mathrm{e}-01$ & 6.09 & $16.1(1.3)$ & $16.2(1.2)$ & $16.0(1.9)$ \\
\hline${ }^{13} \mathrm{CO}$ & 1 & - & $475_{-325}^{+\infty}$ & - & $7.17 \mathrm{e}+09$ & 2.97 & - & $15.6(1.3)$ & - \\
\hline HD 100546 & 2 & $18.50_{-0.4}^{+0.9}$ & $875_{-275}^{+275}$ & $1.41 \mathrm{e}-01$ & $2.29 \mathrm{e}+00$ & 10.24 & $18.1(0.9)$ & $16.5(1.3)$ & $17.2(3.0)$ \\
\hline HD 100546 & 3 & - & $750_{-75}^{+175}$ & $5.50 \mathrm{e}-01$ & $5.13 e+03$ & 4.41 & $16.5(1.3)$ & $16.4(2.6)$ & $15.7(2.4)$ \\
\hline HD 100546 & 4 & - & $1125_{-200}^{+575}$ & $3.15 \mathrm{e}-02$ & $4.02 \mathrm{e}+03$ & 0.84 & $13.8(3.0)$ & $17.0(2.0)$ & $17.5(3.0)$ \\
\hline HD 100546 & 5 & - & $1225_{-125}^{+200}$ & $8.03 \mathrm{e}-02$ & $1.36 \mathrm{e}+04$ & 4.17 & $19.5(3.7)$ & - & $19.3(4.2)$ \\
\hline HD 97048 & 1 & $18.40_{-1.4}^{+\infty}$ & $350_{-125}^{+350}$ & $3.69 \mathrm{e}+00$ & $7.88 \mathrm{e}+04$ & 1.38 & $18.2(2.4)$ & $16.0(1.4)$ & $16.1(1.8)$ \\
\hline${ }^{13} \mathrm{CO}$ & 1 & - & $-^{-123}$ & - & - & 6.82 & $15.5(0.9)$ & $19.2(2.4)$ & - \\
\hline HD 97048 & 2 & $18.90_{-0.4}^{+\infty}$ & $975_{-550}^{+\infty}$ & - & $2.94 \mathrm{e}+01$ & 2.06 & $18.1(0.9)$ & $17.3(1.8)$ & - \\
\hline HD 97048 & 3 & $19.40_{-12}^{+\infty .4}$ & $1125_{-350}^{+\infty}$ & - & $1.36 \mathrm{e}+00$ & 8.01 & $19.7(0.5)$ & $19.9(2.9)$ & $17.7(0.3)$ \\
\hline HD 97048 & 4 & $-\quad-1.2$ & $1075_{-325}^{+\infty}$ & - & $2.22 \mathrm{e}+05$ & 4.67 & $23.4(2.9)$ & $19.9(4.5)$ & $18.6(3.6)$ \\
\hline HD 97048 & 5 & - & $1325_{-475}^{+600}$ & $3.11 \mathrm{e}-02$ & $1.38 \mathrm{e}+06$ & 1.39 & $16.4(1.7)$ & $20.5(4.3)$ & 26.1(6.9) \\
\hline HD 179218 & 1 & $17.20_{-\infty}^{+0.4}$ & $1100_{-375}^{+475}$ & $3.27 \mathrm{e}-01$ & $4.28 \mathrm{e}+00$ & 0.67 & $16.0(5.1)^{*}$ & $13.7(10.3)^{*}$ & $17.7(5.4)$ \\
\hline${ }^{13} \mathrm{CO}$ & 1 & $18.70_{-\infty}^{+0.6}$ & $500_{-250}^{+350}$ & $1.18 \mathrm{e}+00$ & $3.35 \mathrm{e}+05$ & 1.29 & $15.0(7.9)^{*}$ & $19.5(1.7)^{*}$ & $19.0(0.9)$ \\
\hline HD 179218 & 2 & $17.80_{-\infty}^{+\infty}$ & $1225_{-375}^{+500}$ & $5.53 \mathrm{e}-02$ & $7.06 \mathrm{e}+00$ & 2.71 & $19.3(0.7)$ & $21.7(2.1)$ & $21.0(4.8)$ \\
\hline HD 179218 & 3 & $20.00_{-1.8}^{+\infty}$ & $900_{-150}^{+\infty}$ & - & $1.07 \mathrm{e}+00$ & 1.09 & - & $20.6(4.6)$ & $22.1(1.3)$ \\
\hline HD 179218 & 4 & - & $1250_{-400}^{+\infty}$ & - & $5.39 \mathrm{e}+04$ & 0.21 & - & $16.6(1.4)$ & $23.2(4.2)$ \\
\hline HD 141569 & 2 & - & $225_{-50}^{+125}$ & $2.11 \mathrm{e}+01$ & $6.71 \mathrm{e}+12$ & 1.73 & $16.1(2.4)$ & $16.3(1.6)$ & - \\
\hline${ }^{13} \mathrm{CO}$ & 1 & $20.50_{-22}^{+\infty}$ & - & - & - & 0.14 & $15.1(2.1)$ & $13.1(1.4)$ & - \\
\hline HD 190073 & 1 & $19.30_{-07}^{+0.2}$ & $500_{-100}^{+225}$ & $4.40 \mathrm{e}+00$ & $1.91 \mathrm{e}+02$ & 13.61 & $15.6(2.6)$ & - & $16.4(2.4)$ \\
\hline${ }^{13} \mathrm{CO}$ & 1 & $19.30_{-0.2}^{+0.1}$ & $575_{-175}^{+170}$ & $1.36 \mathrm{e}+00$ & $6.45 e+02$ & 11.12 & $12.7(0.5)$ & $12.3(2.1)$ & $11.5(2.4)$ \\
\hline HD 190073 & 2 & $19.80_{-13}^{+\infty .2}$ & $2500_{-1600}^{+\infty}$ & - & $7.00 \mathrm{e}-01$ & 5.98 & $15.5(3.1)$ & $15.8(1.0)$ & $17.3(3.7)$ \\
\hline HD 98922 & 1 & $17.70_{-0.1}^{+0.3}$ & $1175_{-125}^{+1600}$ & $1.06 \mathrm{e}+01$ & $2.05 \mathrm{e}+01$ & 14.18 & $18.1(0.9)$ & $18.6(8.8)$ & $26.1(4.1)$ \\
\hline${ }^{13} \mathrm{CO}$ & 1 & $19.70_{-0.3}^{+0.1}$ & $475_{-275}^{+\infty}$ & - & $5.40 \mathrm{e}+08$ & 2.16 & $16.5(0.2)$ & $17.8(1.8)$ & $21.3(1.7)$ \\
\hline HD 98922 & 2 & - & $575_{-75}^{+\infty}$ & - & $3.86 \mathrm{e}+02$ & 0.87 & $26.7(1.1)$ & $22.5(5.1)$ & $29.6(5.2)$ \\
\hline HD 95881 & 1 & $17.80_{-0.3}^{+0.3}$ & $875_{-200}^{+400}$ & $3.40 \mathrm{e}-01$ & $4.09 \mathrm{e}+00$ & 16.42 & $34.4(4.8)$ & $29.2(6.9)$ & $47.2(4.4)$ \\
\hline${ }^{13} \mathrm{CO}$ & 1 & $-\quad-0.3$ & $400_{-250}^{+260}$ & $5.64 \mathrm{e}-01$ & $1.84 \mathrm{e}+11$ & 1.04 & 22.9(3.9) & $24.5(4.0)$ & $17.5(12.4)$ \\
\hline $\mathrm{R} \mathrm{CrA}$ & 1 & - & $1225_{-850}^{+\infty}$ & - & $2.33 \mathrm{e}+03$ & 1.36 & $10.3(0.5)^{*}$ & $11.8(0.6)^{*}$ & $18.8(3.9)$ \\
\hline HD 135344B & 1 & $17.50_{-0.2}^{+0.1}$ & $800_{-75}^{+100}$ & $2.78 \mathrm{e}-01$ & $8.84 \mathrm{e}-01$ & 5.26 & $16.6(2.6)$ & $12.1(1.5)$ & $27.7(3.4)$ \\
\hline HD 150193 & 1 & $16.00_{-\infty}^{+1.9}$ & $1750_{-925}^{+250}$ & $1.75 \mathrm{e}-01$ & $5.84 \mathrm{e}+00$ & 5.77 & $54.1(1.8)$ & - & $60.0(2.2)$ \\
\hline
\end{tabular}

Notes. Errors reported are within the $1 \sigma$ confidence intervals. When the reported error exceeds one border of the explored parameter space, we mark this with " $\infty$ ". Unconstrained quantities are marked with "-" (cf. Figs. A.1 and A.2). In the case of an unconstrained upper (lower) temperature limit, we also omit the upper (lower) limit on the emitting surface. The FWHM value of transitions marked with a star ${ }^{(*)}$ is affected by imperfect telluric correction and/or absorption.

\subsection{CO rotational temperatures}

We show the best fit rotational temperatures in Table 3 and show the corresponding Boltzmann plots in Fig. 3. The temperature errors reported in Table 3 represent a $1 \sigma$ deviation of the best fit value. The complete best fit contours are shown in Fig. A.1.

For HD 101412, we are unable to determine $T_{\text {rot }}$ as individual lines are heavily blended, but since multiple vibrational bands of both $\mathrm{CO}$ fundamental and 1 st overtone emission are detected we estimate a lower limit to $T_{\text {rot }}$ of $2000 \mathrm{~K}$. This value is in agreement with the temperature of $2500 \mathrm{~K}$ found for the $\mathrm{CO}$ emission in HD 101412 by Cowley et al. (2012).

Some trends can be seen. First, the derived temperatures for the $\mathrm{CO}$ temperatures in all disks but HD 141569 is above $500 \mathrm{~K}$, and on average around $1000 \mathrm{~K}$. Second, the ${ }^{12} \mathrm{CO}$ temperatures are higher than the ${ }^{13} \mathrm{CO}$ temperatures, except for HD 190073, where they are similar.

\subsubsection{CO vibrational temperatures}

We show the vibrational diagrams in Fig. 4 and summarize our results in Table 4. To investigate the dominant excitation mechanism of the $\mathrm{CO}$ gas we compare the vibrational
Table 4. Vibrational temperatures and the averaged rotational temperatures of combined vibrational bands for sources with at least $v_{\text {up }}=1,2$ detected.

\begin{tabular}{llllll}
\hline \hline Target & $n_{\text {vib }}$ & $\begin{array}{l}T_{\text {rot }} \\
{[\mathrm{K}]}\end{array}$ & $\begin{array}{l}\text { Error } \\
{[\mathrm{K}]}\end{array}$ & $\begin{array}{l}T_{\text {vib }} \\
{[\mathrm{K}]}\end{array}$ & $\begin{array}{l}\text { Error } \\
{[\mathrm{K}]}\end{array}$ \\
\hline HD 100546 & 5 & 1080 & $+180,-130$ & 6400 & 2100 \\
HD 97048 & 5 & 1330 & $+\infty,-580$ & 2100 & 330 \\
HD 179218 & 5 & 1250 & $+400,-300$ & 6500 & 2700 \\
HD 190073 & 2 & 1130 & $+\infty,-450$ & 900 & 90 \\
HD 98922 & 2 & 1230 & $+230,-180$ & 1300 & 180 \\
\hline
\end{tabular}

Notes. See also Fig. 4. Errors reported marked with $\infty$ have $1 \sigma$ best fit solutions that exceed the parameter space of the grid of models.

and rotational temperatures of all sources with detections of at least the $v_{\mathrm{u}}=2$ emission. We find that the distribution of $\mathrm{CO}$ molecules in the group II (self-shadowed) disks over the vibrational bands is consistent with the temperature derived from the rotational transitions, i.e. in agreement with collisions being the dominant excitation mechanism. The higher vibrational bands in the group I disks, however, show an overpopulation compared to the numbers expected for gas in LTE. 
Summarizing, we find (1) that $T_{\text {vib }}>T_{\text {rot }}$ for the flaring disks and (2) that $T_{\text {vib }} \approx T_{\text {rot }}$ for the self-shadowed disks.

\subsection{Kinematics}

Line width: the shape of an emission line originating from a circumstellar disk is determined by the mass of the central star, the disk inclination, and the radial location of the emitting gas. Thus, a spectrally resolved emission line is an indicator for the distribution of the emitting gas throughout the disk. Comparing the line shape and width both for the rotational transitions within a vibrational band $(\Delta E \approx 10-100 \mathrm{~K})$, and between the vibrational bands $(\Delta E \approx 1000 \mathrm{~K})$ is a useful exercise to compare the location of the emitting gas over a large range of excitation conditions.

As a proxy for line shape, we have measured the FWHM of each ro-vibrational line as function of $J_{\text {up }}$ (and thus excitation energy). We show the average FWHM values for low $\left(J_{\text {up }} \leq 10\right)$, medium $\left(10<J_{\text {up }} \leq 20\right)$ and high $\left(J_{\text {up }}>20\right)$ in Table 3 . The FWHM of the low $\left(J_{\text {up }} \leq 15\right)$ rotational transitions is constant within our errors, and we construct composite line profiles for each detected vibrational band of these lines with sufficient $\mathrm{S} / \mathrm{N}$, to compare the line shapes. To construct these composite profiles we center each emission line at its line center, re-grid it on a velocity grid, subtract the continuum, and scale the line maximum to 1 . We show the composite profiles in Fig. 5. The line profiles of HD 100546 and HD $97048 v=3-2$, and HD 150193, HD 179218 and R CrA $v=1-0$, have been made using the high $\left(J_{\text {up }}>20\right)$ ro-vibrational transitions, because no suitable low $J$ lines were available.

One would expect that line width correlates with the energy needed to excite the line - the higher excitation lines originating from closer to the star and so showing broader lines. Surprisingly, only two stars in our sample, HD 98922 and HD 135344B, show this trend (Table 3). In HD 100546, there is no such change in line width between the different vibrational bands, but the line shape of the ${ }^{12} \mathrm{CO}$ transitions become more flat-topped with increasing excitation levels. In the remaining stars of our sample we find no correlation between line excitation and line shape, nor between self-shadowed and flaring disks. For all stars with self-shadowed disks the ${ }^{13} \mathrm{CO}$ line width (FWHM) is smaller than that of the corresponding ${ }^{12} \mathrm{CO}$ lines. This is not seen in the flaring disks. We will return to these trends in Sect. 7.1.2.

Radial velocity: to determine the radial velocity of the $\mathrm{CO}$ emission we fit a two-dimensional polynomial to the offset between the telluric absorption lines in the spectra and a HITRAN synthetic atmosphere model (Rothman et al. 1992), correct for the barycentric velocity, and measure the centroid of the emission line. We list these values in Table 2. Although missing error estimates for some stellar radial velocities from the literature make a quantitative interpretation for individual targets difficult, on average the observed $\mathrm{CO}$ emission line velocities appear to be similar to or slightly blue shifted from the stellar radial velocities from the literature. A slight blue-shift of the observed $\mathrm{CO}$ emission would be in line with a contribution to the CO line emission from a slowly rotating disk wind (cf. Sect. 6; Bast et al. 2011; Pontoppidan et al. 2011).

\subsection{CO first overtone emission}

We searched for first overtone CO emission in all our targets except HD 98922, HD 104237 and HD 142666, for which no spectra covering the $2.2-2.3 \mu \mathrm{m}$ range are available. We detect the $v=3-1$ band head and confirm the presence of CO $v=2-0$ overtone emission in the disk around HD 101412 reported by Cowley et al. (2012) and Ilee et al. (2014). We detect no $\mathrm{CO}$ overtone emission in any of the other targets which exhibit $\mathrm{CO}$ fundamental emission, which is in agreement with the $7 \%$ detection rate of $\mathrm{CO}$ overtone emission from disks around HAeBe stars by Ilee et al. (2014). For these disks we define $3 \sigma$ upper limits using a line width of $20 \mathrm{~km} \mathrm{~s}^{-1}$ (a width typical for the fundamental $\mathrm{CO}$ emission) and continuum fluxes as published in the 2MASS catalog (Skrutskie et al. 2006). We do detect first overtone $\mathrm{CO}$ foreground absorption towards $\mathrm{R} \mathrm{CrA}$ (cf. Sect. 5.6). See Table B.2 and Fig. 2 for the spectra, line fluxes and upper limits.

\subsection{Spatially resolved $\mathrm{CO}$ and continuum emission}

The $4.6 \mu \mathrm{m}$ continuum emission is spatially resolved in HD 97048 and HD 100546 (see van der Plas et al. 2009 for details) and, marginally, in HD 179218. The continuum emission of HD 179218 has a FWHM of $0.209^{\prime \prime} \pm 0.005^{\prime \prime}$, or a deconvolved, deprojected size of $0.078^{\prime \prime} \pm 0.026^{\prime \prime}$ compared to the FWHM of the unresolved PSF of the telluric standards observed directly before $\left(0.194^{\prime \prime} \pm 0.005^{\prime \prime}\right)$ and after $\left(0.192^{\prime \prime} \pm 0.006^{\prime \prime}\right)$ the observations.

The CO emission in HD 97048, HD 100546 and HD 141569 is spatially resolved and shows equally resolved red- and blueshifted components on spatially opposite sides of the star, compatible with a disk in Keplerian rotation. To calculate the spatial extent of the $\mathrm{CO}$ emission we sample and subtract the continuum on the red side of the line after scaling it to the intensity of the telluric spectrum at each corresponding spectral bin. We define the outer radius of the $\mathrm{CO}$ emission as the maximum extent of the $2 \sigma$ contour of the continuum subtracted CO $v_{\mathrm{u}}=2$ R05 emission line on the position-velocity diagram and the error as 1 pixel $\left(0.086^{\prime \prime}\right)$. Using this procedure, in our spectra the CO emission is spatially resolved up to radii of $0.40^{\prime \prime} \pm 0.09^{\prime \prime}, 0.34^{\prime \prime} \pm 0.09^{\prime \prime}$ and $0.30^{\prime \prime} \pm 0.09^{\prime \prime}$ for HD 97048, HD 100546 and HD 141569, respectively. For HD 97048 this is the first published spatial extent of the CO emission. The values for HD 100546 and HD 141569 are smaller but consistent with the spatial CO extent published previously, which was based on similar or higher SN spectra (cf. Goto et al. 2006, 2012; van der Plas et al. 2009; Hein Bertelsen et al. 2014).

The $\mathrm{CO}$ emission in $\mathrm{R} \mathrm{CrA}$ is only resolved in one spatial direction, and blueshifted $10 \mathrm{~km} \mathrm{~s}^{-1}$ with respect to the surrounding Corona Australis molecular cloud (cf. Sect. 5.6). This suggests the $\mathrm{CO}$ emission in the $\mathrm{R} \mathrm{CrA}$ spectrum is likely to originate from an outflow. We note that the presence of an outflow is also suggested from the bow-shocks seen in shocked $\mathrm{H}_{2}$ emission in the immediate vicinity of R CrA (Kumar et al. 2011). The detected CO emission is spatially unresolved in the data for all other targets.

\subsection{CO absorption}

We detect CO absorption in the spectra of R CrA and HD 97048. In $\mathrm{R} \mathrm{CrA}$, we detect the ${ }^{12} \mathrm{CO} v=0-1, v=0-2$, and ${ }^{13} \mathrm{CO} v=$ $0-1$ bands in the spectrum. We show two absorption lines, together with the line widths and a Boltzmann plot in Fig. 6.

The absorption lines in $\mathrm{R} \mathrm{CrA}$ are spectrally resolved, and the radial velocity of the ${ }^{12} \mathrm{CO} v=1-0$ emission is blue shifted $10 \mathrm{~km} \mathrm{~s}^{-1}$ with respect to the ${ }^{12} \mathrm{CO} v=0-1, v=0-2$, and ${ }^{13} \mathrm{CO} v=0-1$ absorption. We calculate a $\mathrm{CO}$ isotopologue ratio of ${ }^{12} \mathrm{CO} v=0-2 /{ }^{13} \mathrm{CO} v=0-1$ towards $\mathrm{R} \mathrm{CrA}$ of 142 , and 


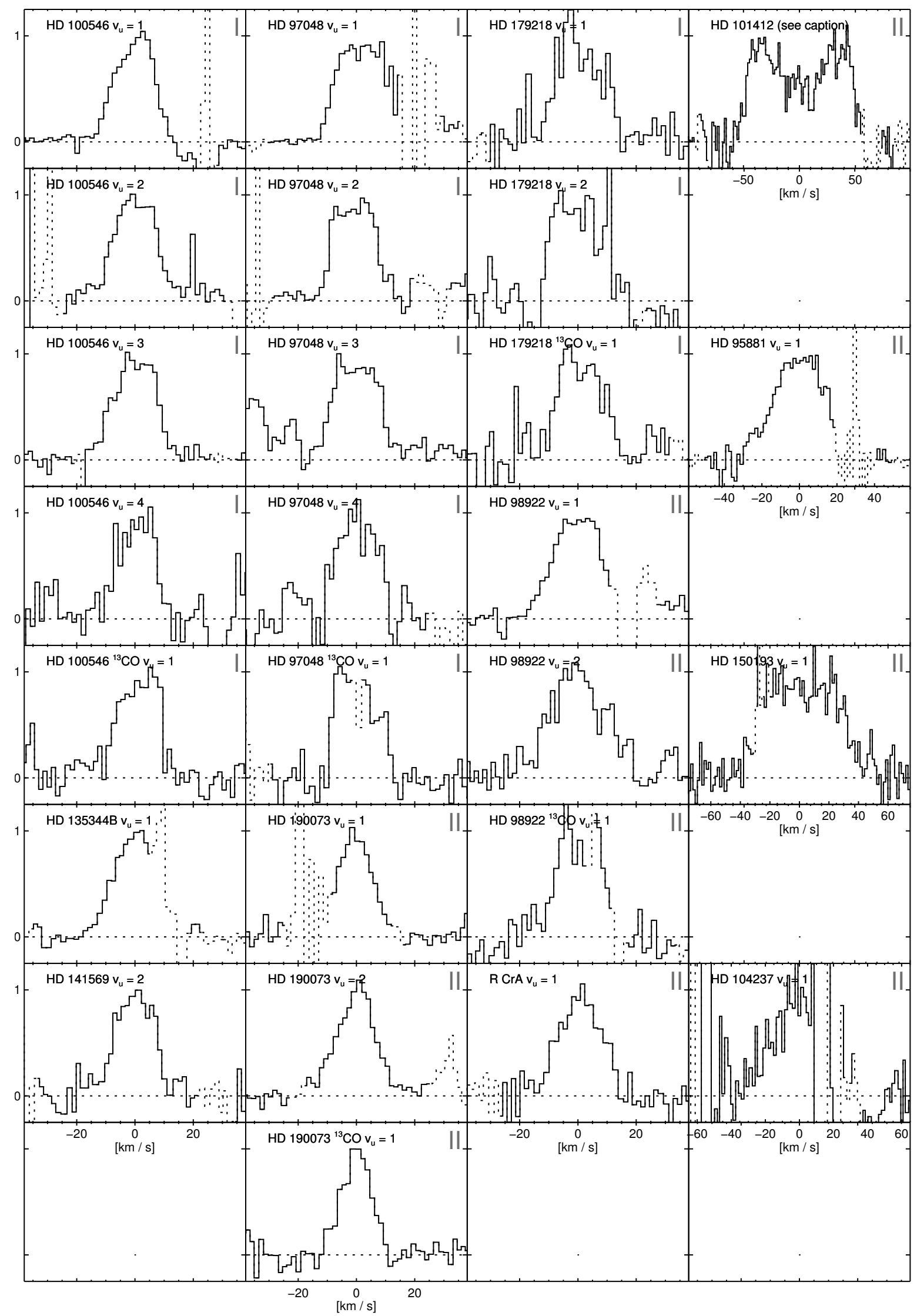

Fig. 5. Averaged, normalized composite line profiles for all detected vibrational bands that have sufficient signal as described is Sect. 5.3. The composite profiles of different isotopologues and vibrational bands of the same source are presented below each other, the disk classification (group I/II) is noted on the top right. The line profile for HD 101412 is a mix of the ${ }^{13} \mathrm{CO} v=1-0 \mathrm{R} 23$ and ${ }^{12} \mathrm{CO} v=4-3 \mathrm{R} 35$ lines. 

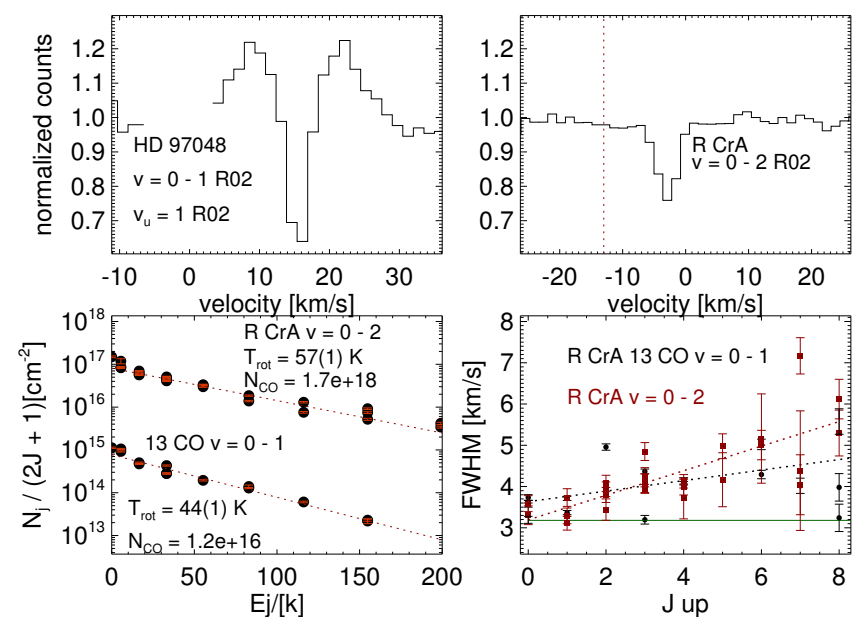

Fig. 6. ${ }^{12} \mathrm{CO} v=1-0 \mathrm{R} 02$ emission and $v=0-1$ absorption in HD 97048 (upper left panel). ${ }^{12} \mathrm{CO} v=0-2 \mathrm{R} 02$ absorption in R CrA (upper right panel), the line center velocity of the ${ }^{12} \mathrm{CO} v=1-0$ emission lines is shown by the red dashed line. The Boltzmann plot (lower left panel) and line widths (lower right panel) for the ${ }^{12} \mathrm{CO} v=0-2$ and ${ }^{13} \mathrm{CO} v 0-1$ ro-vibrational absorption lines in the spectrum of $\mathrm{R} \mathrm{CrA}$ (notation similar to Fig. 3). The vertical green line in the bottom right panel at $3.18 \mathrm{~km} \mathrm{~s}^{-1}$ shows the spectral resolution of our observations.

a temperature ratio of $57 \mathrm{~K} / 44 \mathrm{~K}=1.3$. These values are comparable to values found toward local molecular clouds (Goto et al. 2003), and are consistent with a scenario in which the absorption lines are formed in the surrounding Corona Australis molecular cloud.

We detect ${ }^{12} \mathrm{CO}$ and ${ }^{13} \mathrm{CO} v=0-1$ absorption lines in the spectrum of HD 97048. The absorption lines in HD 97048 are unresolved and blended with emission lines, making it difficult to reliably determine the line strengths. The radial velocity of the absorption and emission lines is similar.

\subsection{Other emission lines}

Except for HD 101412, all targets with detected CO emission also show the $\mathrm{H}$ I recombination lines of $\operatorname{Pf} \beta(4.654 \mu \mathrm{m})$ and $\mathrm{Hu} \epsilon(4.672 \mu \mathrm{m})$ in emission. The line widths of the H I recombination lines are much wider than those of the CO lines (Fig. 1), and has been used by Salyk et al. (2013) as an accretion tracer. The origin of these infrared $\mathrm{H}$ I emission lines is likely identical to the optical H I emission, which is also believed to be linked closely to accretion (e.g. Calvet et al. 2004; Garcia Lopez et al. 2006; Medigutia et al. 2011). The H I lines we detect here are consistent with those presented in the literature so far. We list the line flux and FWHM values in Table B.3 and refrain from discussing these in more detail in the current paper.

We detect the neutral Na I D doublet (2.206 and $2.209 \mu \mathrm{m}$, $4 \mathrm{p}^{2} \mathrm{P}^{0}-4 \mathrm{~s}^{2} \mathrm{~S}$ ) in emission in the spectrum of HD 190073, with a FWHM of $34 \pm 3 \mathrm{~km} \mathrm{~s}^{-1}$, and a $2.206 \mu \mathrm{m} / 2.209 \mu \mathrm{m}$ line ratio of $\approx 2$. The Na I radial velocity in HD 190073 is $-3 \pm 3 \mathrm{~km} \mathrm{~s}^{-1}$, and is in agreement with the radial velocity of the central star and the $\mathrm{CO}$ emission. The low first ionization potential of neutral sodium $(5.1 \mathrm{eV}, 243 \mathrm{~nm})$, makes a non-thermal excitation mechanism such as fluorescent pumping by $330.3 \mathrm{~nm}$ photons (Thompson \& Boroson 1977), or ionization and subsequent recombination likely. This doublet is more often detected in earlytype high-luminosity stars (McGregor et al. 1988), as well as in EXor stars in concert with CO first overtone $v=2-0$ emission, and in those cases is explained by disk emission (Lorenzetti et al. 2009). We note that CO first overtone emission is not detected in HD 190073.

HD 98922 has an un-identified emission line at $4.7485 \mu \mathrm{m}$, similar in line shape and line flux $\left(5.5 \times 10^{-14} \mathrm{erg} \mathrm{cm}^{-2} \mathrm{~s}^{-1}\right)$, but with a FWHM of $14 \mathrm{~km} \mathrm{~s}^{-1}$ slightly narrower than the CO emission lines.

\subsection{Summary of observed trends in the CO emission}

We detect ro-vibrational fundamental ${ }^{12} \mathrm{CO}$ emission in 12 out of 13 surveyed HAeBe stars. The temperature of the $\mathrm{CO}$ gas in these objects varies, with a few exceptions, between 500 and $2000 \mathrm{~K}$. We also detect ${ }^{13} \mathrm{CO}$ emission in 8 of these objects, with temperatures between 200 and $1050 \mathrm{~K}$, all lower than or similar to the ${ }^{12} \mathrm{CO}$ rotational temperature in the same disks. There is a correlation between line shape and excitation temperature in at least two disks. However, this correlation between line shape and excitation temperature is absent in the higher vibrational bands in the three flaring disks. In these disks, the CO fundamental emission is excited high up the vibrational ladder (at least up to $v=5-4$ ), but no CO overtone emission is detected. We have spatially resolved the $4.6 \mu \mathrm{m}$ continuum emission in all flaring disks and the $\mathrm{CO}$ emission in the two out of three flaring disks, with outer radii for the $\mathrm{CO}$ emission of $\approx 70$ au for HD 97048 and 35 au for HD 100546. We detect highly excited $\mathrm{CO}$ fundamental and $\mathrm{CO}$ overtone emission in the disk around HD 101412, and CO absorption toward R CrA and HD 97048.

\section{Location of the $\mathrm{CO}$ emitting gas}

Carbon monoxide fundamental emission appears not to be a direct proxy for gas in the disks around HAeBe stars. The $\mathrm{CO}$ emission around group I sources originates from larger radii than around group II sources, but in both types of disks there is circumstellar gas present closer to the central star. There is no correlation between the $4.77 \mu \mathrm{m}$ continuum flux and radial CO location, ruling out contrast effects.

We wish to trace the $\mathrm{CO}$ as close as possible to its inner radius. Because of the quality of our data, it is difficult to assess where the line ends and the continuum starts. Therefore, we use the Half Width at $10 \%$ of the line maximum (HW10M). We define the onset of the $\mathrm{CO}$ emission $\left(R_{\mathrm{CO}, 10 \%}\right)$ using this $\mathrm{HW} 10 \mathrm{M}$, the assumption of Keplerian rotation, and the stellar parameters from Table 1. In case of missing error estimates, we use an error of $\pm 10 \%$ for the stellar mass, and $\pm 10^{\circ}$ for the inclination.

We compare the $R_{\mathrm{CO}, 10 \%}$ with the naively expected cut-off of the inner disk, the dust sublimation radius at 1500 and $2000 \mathrm{~K}$ and, when available, the radius of the inner disk rim as traced by $2 \mu \mathrm{m}$ interferometry. We calculate the dust evaporation radius following

$R_{\text {subl }}=0.035 \sqrt{Q_{\mathrm{R}}} \sqrt{\frac{L_{\star}}{L_{\odot}}}\left(\frac{1500}{T_{\text {subl }}}\right)^{2}$

with $Q_{\mathrm{R}}$ the ratio of the dust absorption efficiencies for radiation at color temperature $T$ of the incident and re-emitted field. For a star of $10000 \mathrm{~K}, \sqrt{Q_{\mathrm{R}}}$ varies between 1 and 4.5 for grain sizes between respectively 1.00 and $0.01 \mu \mathrm{m}$ (cf. Fig. 2 of Monnier \& Millan-Gabet 2002). For our calculations we adopt $\sqrt{Q_{\mathrm{R}}}=2$, but we note that the choice for $\sqrt{Q_{\mathrm{R}}}$ used in the literature for $\mathrm{T}$ Tauri stars varies between 1 and 2 , and that this value increases with the color temperature of the central star. We show the 
Table 5. Comparison of $R_{\mathrm{CO}, 10 \%}$ to the location of the inner dust disk as calculated from the dust sublimation radius and, where available, as measured with IR interferometry.

\begin{tabular}{llllll}
\hline \hline Name & $R_{\mathrm{CO}, 10 \%}$ & $R_{\text {subl }}^{1500 \mathrm{~K}}$ & $R_{\text {subl }}^{2000 \mathrm{~K}}$ & $R_{\text {dust }}^{\mathrm{in}, 2 \mu \mathrm{m}}$ & $L_{6.2 \mu \mathrm{m}}^{\mathrm{PAH}} / L_{*}$ \\
\hline Group I & {$[\mathrm{au}]$} & {$[\mathrm{au}]$} & {$[\mathrm{au}]$} & {$[\mathrm{au}]$} & \\
\hline HD 100546 & $6.8^{ \pm 1.6}$ & 0.45 & 0.25 & $0.26^{e}$ & $1.30 \pm 0.13(-3)$ \\
HD 97048 & $10.1^{ \pm 1.8}$ & 0.36 & 0.20 & & $1.29 \pm 0.01(-3)$ \\
HD 179218 & $9.2^{ \pm 1.5}$ & 0.61 & 0.34 & $>3.1^{a}$ & $1.65 \pm 0.17(-3)$ \\
\hline Group II & & & & & \\
\hline HD 101412 & $0.6^{ \pm 0.1}$ & 0.35 & 0.20 & & $2.57 \pm 0.05(-4)$ \\
HD 190073 & $1.9^{ \pm 2.8, \dagger}$ & 0.64 & 0.36 & $0.62^{b}$ & $9.61 \pm 2.80(-5)$ \\
HD 98922 & $4.2^{ \pm 2.2, \dagger}$ & 2.09 & 1.18 & $1.25^{c}$ & n.s. \\
HD 95881 & $1.9^{ \pm 0.6, \ddagger}$ & 0.33 & 0.18 & $0.37^{d}$ & $9.53 \pm 0.08(-4)$ \\
HD 150193 & $0.5^{ \pm 0.2}$ & 0.28 & 0.15 & $0.71^{a}$ & $<5.3(-5)$ \\
HD 104237 & $0.3^{ \pm 0.5, \dagger}$ & 0.41 & 0.23 & $0.29^{c}$ & $<5.3(-6)$ \\
\hline Not classified & & & & & \\
\hline HD 141569 & $10.4^{ \pm 1.9, \dagger}$ & 0.25 & 0.14 & & $8.70 \pm 0.10(-5)$ \\
HD 135344B & $0.4^{ \pm 0.2}$ & 0.22 & 0.13 & $0.2^{f}$ & $2.74 \pm 0.12(-4)$ \\
\hline
\end{tabular}

Notes. The references for the $2 \mu \mathrm{m}$ inner rim sizes in the fifth column are: ${ }^{(a)}$ Monnier et al. (2006), IOTA, $H$ band. ${ }^{(b)}$ Eisner et al. (2009) (Keck, $K$ band). ${ }^{(c)}$ Kraus et al. (2008), VLTI/AMBER, $K$ band. ${ }^{(d)}$ Verhoeff et al. (2010), VLTI/AMBER, $K$ band. ${ }^{(e)}$ Benisty et al. (2010), VLTI/AMBER, $K$ band, $(f)$ Carmona et al. (2014), VLTI/PIONIER $H$ band. PAH to luminosity ratios in the last column are adapted from Acke et al. (2010) and Acke \& van den Ancker (2004). The upper limits are $3 \sigma, a(b)$ represents $a \times 10^{b}$, and n.s. means no spectrum is available. To calculate the error on $R_{\mathrm{CO}, 10 \%}$ for sources with missing error estimates we adopt a $10 \%$ error for the values of the stellar mass ${ }^{(\dagger)}$ and the inclination ${ }^{(\dot{*})}$.

HW10M radii, the dust sublimation radii for 1500 and $2000 \mathrm{~K}$, the radius of the inner rim as traced by $2 \mu \mathrm{m}$ interferometry, and the PAH-to-stellar-luminosity ratio in Table 5.

Inspection of Table 5 shows that the inner rim sizes determined from the hot dust loosely agree with a dust sublimation temperature between 1500 and $2000 \mathrm{~K}$, and that the $\mathrm{CO}$ emission starts at the largest radii for flaring disks. In the group I (flaring) disks, the $\mathrm{CO}$ emission starts far beyond the calculated dust sublimation radius, at $\approx 16-48 R_{\text {subl }}$. The disks with a group II (selfshadowed) dust disk but still flaring gas (HD 95881, HD 98922 and HD 101412, cf. Sect. 7.3.2), have intermediate CO inner radii, and the $\mathrm{CO}$ onset of the self-shadowed disks without flaring gas disks is approximately similar to $R_{\text {subl }}$.

The outlier in this picture is HD 190073, but we argue that it falls within the picture described, given the uncertainty in its stellar parameters. For HD 190073, the error on the inclination of $23_{-23}^{+15}$ degrees is large. Eisner et al. (2004) note that their data for HD 190073 is also consistent with an almost face-on inclination. If we adopt an inclination of $12^{\circ}$, The best fit $\mathrm{CO}$ inner radius becomes $0.35 \mathrm{au}$, the distance of the central star where $T_{\text {dust }}=2000 \mathrm{~K}$.

\subsection{Keplerian fits to the line profiles}

To investigate the spatial distribution of the $\mathrm{CO}$ emission as well as to find out to what extent the narrow $\left(0.2^{\prime \prime}\right)$ spectrograph slit obscures the disk and influences the line profiles, we create a basic model of the $\mathrm{CO}$ emission. In this model, the gas is in Keplerian orbit in a flat disk with known inclination and PA around a star with known stellar mass and distance. The intensity of the emission decreases as $I(R)=I_{\text {in }}\left(\frac{R}{R_{\text {in }}}\right)^{-\alpha}$, with $\alpha=2$, $I_{\text {in }}$ the intensity at the inner radius $R_{\text {in }}$, and $R$ the radial distance from the star. $R_{\text {in }}$ and $R_{\text {out }}$ are the only free parameters in this model. The simulated disk is then convolved with the observed telluric PSF, and we project the slit over the simulated disk and calculate the resulting line profile. HD 104237 and HD 98922 have no known disk PAs and we assume those to be 0 degrees. Since the $\mathrm{CO}$ emission from those sources is not spatially resolved we assume no emission is cut off by the slit and thus that the observed line profile will not be a function of the slit PA. We show our best fits together with a sketch of the best fit disk+slit system in Fig. 7. Using our simple Keplerian model, we need outer disk radii $\geq 35$ au for 8 of the 12 targets.

\subsection{A comparison to CO emission from T Tauri stars}

$\mathrm{CO}$ emission around $\mathrm{T}$ Tauri stars, the less massive siblings of $\mathrm{HAeBe}$ stars, is often detected at much smaller radii than in HAeBe stars. Najita et al. (2003) find an average FWHM of $70 \mathrm{~km} \mathrm{~s}^{-1}$ for 12 surveyed T Tauri stars, corresponding to inner radii of $0.02-0.05 \mathrm{au}$, and conclude that most emission comes from (close to) the co rotation radius. Salyk et al. (2009) study 14 transitional (circumstellar disks with an optically thick outer zone but an inner region significantly depleted of small dust grains, of which 13 T Tauri stars and one HAeBe star) disks, and find that the disks with partially depleted inner disks most often have $\mathrm{CO}$ that extends to rather small ( $\leq 1 \mathrm{au}$ ) radii, but compared to "classical" disks the CO emission radii are larger than that expected for dust sublimation.

$\mathrm{CO}$ emission in $\mathrm{T}$ Tauri stars thus probes distances as close as the co-rotation radius, which is also the case for most of the group II sources in our sample. Compared to T Tauri stars, $\mathrm{CO}$ emission around $\mathrm{HAeBe}$ group I stars originates from larger radii, analogous to the $\mathrm{T}$ Tauri stars with partially depleted inner disks.

\section{Discussion}

\subsection{CO excitation mechanisms}

Our isothermal slab model successfully reproduces the emission characteristics in all but 3 sources. Only for the group I sources (HD 100546, HD 97048 and HD 179218) do we detect a significant deviation between the rotational and vibrational temperatures. For those sources, we conclude that LTE excitation alone cannot be responsible for the $\mathrm{CO}$ excitation.

One other process that could play a role in $\mathrm{CO}$ excitation in these disks is the direct radiative pumping of the $\mathrm{CO}$ molecule by IR and UV photons. UV pumping (Krotkov et al. 1980) is the process where UV radiation from the star or accreting material pumps $\mathrm{CO}$ in the ground electronic state to an excited electronic state, which then decays back into an excited vibrational band in the ground electronic state. The effect of UV pumping is to redistribute $\mathrm{CO}$ molecules over a large range of vibrational bands. The relative populations of these bands, and thus the vibrational temperature, is equal to or lower than the black body temperature of the stellar UV field (depending on the dilution of the stellar UV field which diminishes the influence of the UV pumping over the LTE de-excitation). IR pumping (Scoville et al. 1980) is the process in which the IR radiation field coming from the star and the local $4.6 \mu \mathrm{m}$ dust continuum directly pumps the fundamental $\mathrm{CO}$ lines. This process is most efficient in pumping the $v_{\mathrm{u}}=1$ lines (as compared to the higher bands), and plays its biggest role in HAeBe disks in the innermost disk. 


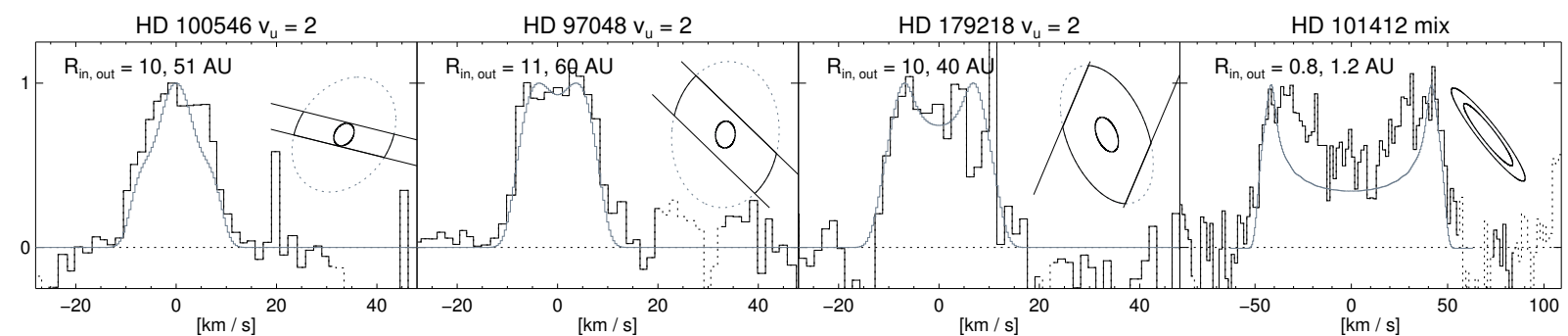

HD $141569 v_{u}=2$ HD $98922 v_{u}=1$ HD $95881 v_{u}=1$
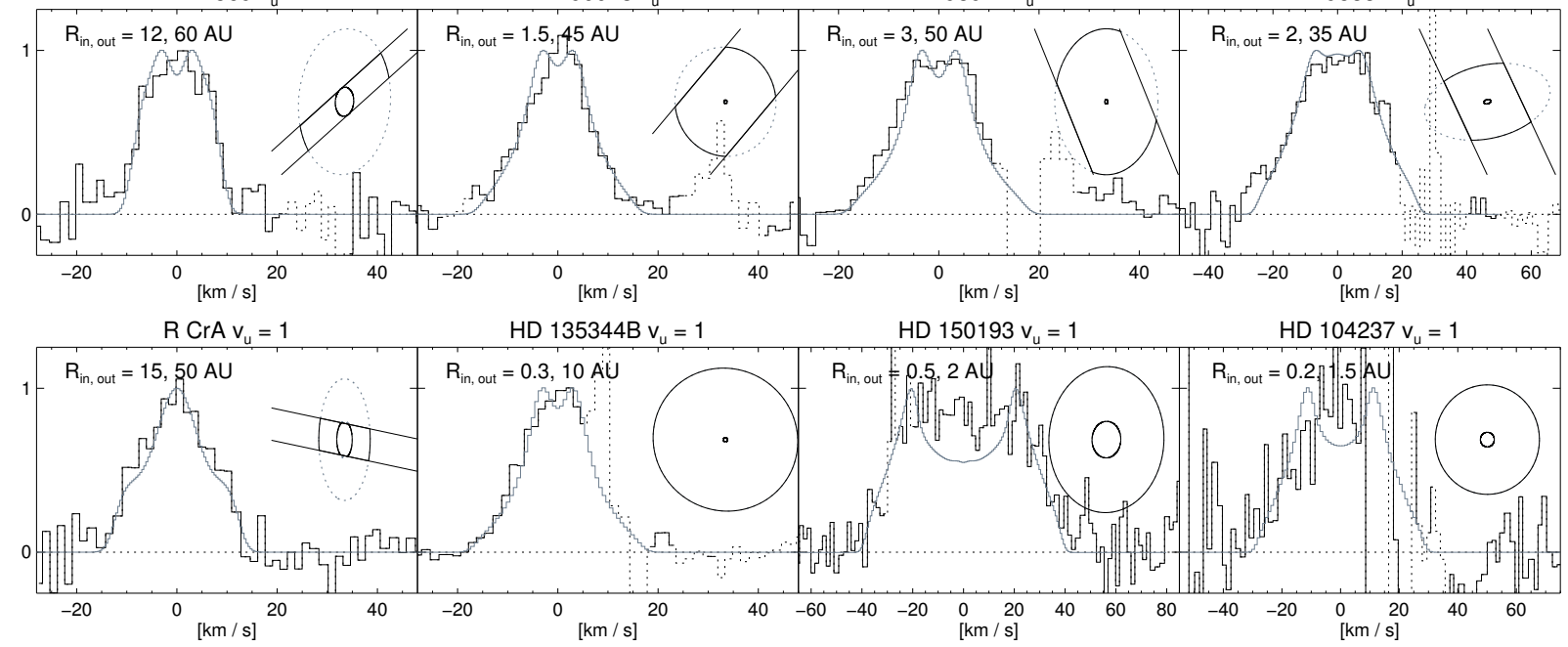

Fig. 7. Normalized composite line profiles (black histogram) and the best model fit (gray line). Also plotted are the respective simulated disk+slit (north is up, east is left), and the best fit CO inner- and outer radius used in the model. The line profile for HD 101412 is a mix of the ${ }^{13} \mathrm{CO} v=1-0$ $\mathrm{R} 23$ and ${ }^{12} \mathrm{CO} v=4-3 \mathrm{R} 35$ lines.

Both processes have been used to explain $\mathrm{CO}$ emission from disks with non-LTE line populations. See e.g. Brittain et al. (2003, 2007, 2009); Brown et al. (2013) for the use of UV pumping and Blake $\&$ Boogert (2004) for the use or IR pumping. The relative contribution of radiative pumping compared to collisions differs on a case by case basis and depends on the local temperature, density and radiation field. In general, if the $\mathrm{CO}$ emission originates from close to the star where temperatures and densities are high, IR pumping (by photons either coming from the star or the local dust continuum) plays a larger role, and mostly for the $v=1-0$ transitions. As the emission radius increases and temperatures and density drop, UV fluorescence and collisional excitation take over. Modeling work by Thi et al. (2013) on $\mathrm{CO}$ excitation in Herbig Ae disks shows that UV fluorescence plays a larger role in disks of lower mass and in disks with an inner hole/gap. Even if UV and/or IR pumping dominate over the collisional excitation of $\mathrm{CO}$ vibrational bands, they are not expected to have a large impact on the rotational temperature of the $\mathrm{CO}$ molecules. The pure rotational collision rates are a factor of 10-100 larger than the ro-vibrational rates, and we expect the rotational levels within each vibrational band to reflect the local (LTE) temperature.

Detailed modeling of each individual source is beyond the scope of this paper. Rather, we generalize the above discussion as follows. We interpret the rotational temperature to reflect the local gas temperature. If the vibrational bands are excited up to a high level, UV fluorescence is most likely the dominant excitation mechanism and we then calculate the vibrational temperature from the vibrational bands above $v_{\mathrm{u}}=1$ of all detected bands (given the possible contribution of LTE excitation or IR pumping to that level). If we only detect CO coming from $v_{\mathrm{u}}=1$ and 2 , and the relative strength of these bands is not compatible with collisional excitation, we interpret this as the presence of IR pumping.

\subsection{1. ${ }^{12} \mathrm{CO}$ temperature}

Rotational temperature: within the confidence intervals given in Table 3, there is a single excitation temperature for the ${ }^{12} \mathrm{CO}$ vibrational bands within each source for all our targets except HD 190073, where the $v_{\mathrm{u}}=2$ excitation temperature is significantly warmer than the $v_{\mathrm{u}}=1$ excitation temperature. Because of the comparable line widths (all disks except HD 98922) and spatial extent (for the flaring disks), we assume the $\mathrm{CO}$ emission coming from all vibrational bands from each disk is emitted from the same surface and calculate one rotational temperature per source for those sources with detections in more than one vibrational band. We do this by summing the $\chi^{2}$ surfaces for all ${ }^{12} \mathrm{CO}$ vibrational bands within each target, and list the resulting rotational temperatures in Table 4.

The low best fit ${ }^{12} \mathrm{CO} v_{\mathrm{u}}=1$ temperature of HD 97048 compared to the averaged temperature necessitates an emitting surface that is a factor of $\approx 1000$ larger then that of the higher vibrational bands in this source. Because the $\mathrm{CO}$ emission in this source has been resolved (van der Plas et al. 2009) we know that the emitting region in this source is similar for all bands. Most likely, our fitting method underestimates the temperature for the ${ }^{12} \mathrm{CO} v_{\mathrm{u}}=1$ emission in HD 97048.

The ${ }^{12} \mathrm{CO} v_{\mathrm{u}}=1$ temperature for HD 190073 is similarly low. For the respective best fit $\left(T_{\text {rot }}, N(\mathrm{CO})\right)$ parameters the emitting surface of the $v_{\mathrm{u}}=1$ emission needs to be a factor of $\approx 1000$ larger than that of the $v_{\mathrm{u}}=2$ emission. This difference shrinks to a factor of $\approx 2$ when considering a shared best fit $T_{\text {rot }}$ and $N(\mathrm{CO})$ obtained by summing the separate $\chi^{2}$ surfaces. 


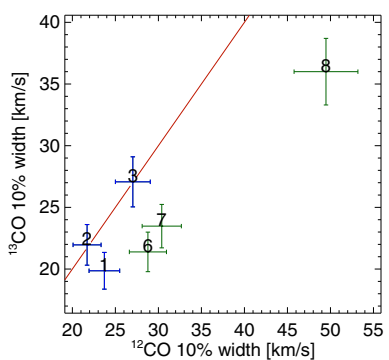

(a)

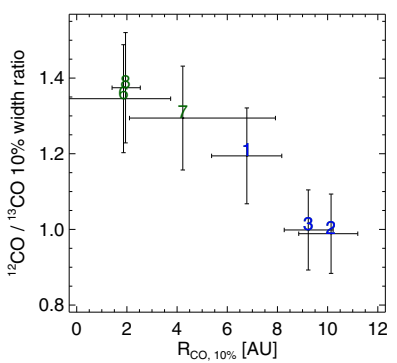

(b)

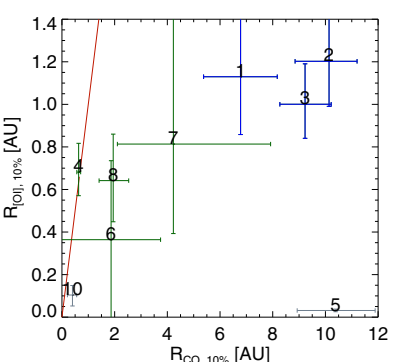

(c)

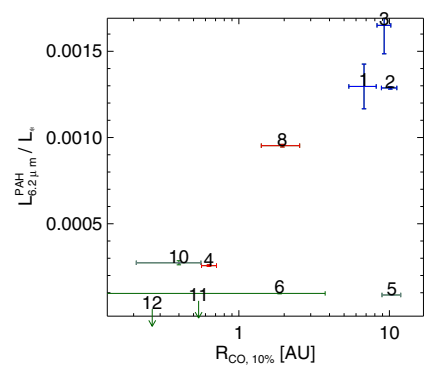

(d)

Fig. 8. From left to right: a) Correlation between the half line width at $10 \%$ of the maximum flux of ${ }^{12} \mathrm{CO}$ and ${ }^{13} \mathrm{CO}$ emission. The $1: 1$ ratio is overplotted with a red line. b) Correlation between the ratio of the $10 \%$ widths and the onset of the ${ }^{12} \mathrm{CO}$ emission as derived from the half width at $10 \%$ of the maximum flux. c) Correlation between the $\mathrm{CO}$ and [O I] inner radii, the red line denotes the 1:1 ratio. d) Correlation between the $\mathrm{CO}$ inner radius and the $L_{6.2}^{\mathrm{PAH}} / L_{*}$ ratio. Sources are named according to Col. 1 from Table 1 . Flaring disks are marked in blue, self-shadowed disks in green and disks with an inner opacity hole are plotted in gray.

The $\mathrm{CO}$ emission is not spatially resolved in this source, and since both best fit surfaces fit within our detection limit it is possible that the $v_{\mathrm{u}}=2$ emission is constrained to a smaller, hotter region compared to the $v_{\mathrm{u}}=1$ emission.

Vibrational temperature: in the vibrational populations of HD 100546 and HD 179218 and, to a lesser extent, in that of HD 97048, we observe a break at $v=2-1$ (cf. Fig. 4). The populations of the higher vibrational bands reflect a temperature higher than the rotational temperature of the gas. Both LTE excitation and IR pumping only affect the lower vibrational bands. UV pumping is required to explain the excitation of the higher vibrational bands. UV pumping alone can not explain the relative over population of the $v_{\mathrm{u}}=1$ level. Both collisional excitation and IR pumping can serve to excite the $\mathrm{CO}$ molecules up to the $v_{\mathrm{u}}=1$ level.

\subsection{2. ${ }^{13} \mathrm{CO}$ temperature}

In all sources in which ${ }^{13} \mathrm{CO}$ was detected, its rotational temperature is similar to or lower than the ${ }^{12} \mathrm{CO}$ rotational temperature. This temperature difference between the isotopes could e.g. be due to opacity effects and a vertical temperature gradient or to a different radial location of the emitting gas. Adding kinematic information can differentiate between the different this degeneracy. In panel a of Fig. 8 we compare the line widths of both isotopes, and show that the ${ }^{13} \mathrm{CO}$ width in the flared disks is within error bars similar to the ${ }^{12} \mathrm{CO}$ width, whereas the ${ }^{13} \mathrm{CO}$ lines in the self-shadowed disks are more narrow, and thus originate farther out. We also plot the ratio of the HW10M of both isotopes against the onset of the ${ }^{12} \mathrm{CO}$ emission (derived from the $\mathrm{HW} 10 \mathrm{M}$ ) in panel $\mathrm{b}$ of the same figure. The magnitude of the radial difference is a function of the onset of the ${ }^{12} \mathrm{CO}$ emission, and this difference decreases for increasing $R_{\mathrm{CO}, 10 \%}$.

This behavior regarding the relative location of the ${ }^{12} \mathrm{CO}$ and ${ }^{13} \mathrm{CO}$ lines can naturally be explained by the fact that ${ }^{13} \mathrm{CO}$ needs a larger column of gas to be detected if these lines are at least partially optically thin. This column is then reached at a larger distance from the star, and so the ${ }^{13} \mathrm{CO}$ rotational temperatures should be lower and the lines should be narrower than ${ }^{12} \mathrm{CO}$, as observed. This difference becomes much smaller for disks with large $\mathrm{CO}$ inner radii, i.e. the flaring disks.

\subsection{CO kinematics}

Our disk + slit model (Fig. 7) yields good fits for the line wings of the disks, but predicts a double peaked emission line where a single peaked line profile is observed for
HD 98922, HD 135344B, HD 141569 and HD 190073. The lack of low projected velocity CO gas in the models of HD 98922 , HD 135344B, HD 141569 and HD 190073 can be remedied by extending the outer radius of the disks. However, because the spectrograph slit truncates large parts of the outer disk, an outer radius $>100$ au is needed to fill in the double peak. Given the quality of our data we expect to spatially resolve the $\mathrm{CO}$ emission in our disks outside $\approx 5$ au for a disk at 100 parsec, but do not do so for all above mentioned targets except HD 141569. This line core issue is also noted for a number of $\mathrm{T}$ Tauri stars by Bast et al. (2011) and Brown et al. (2013). Pontoppidan et al. (2011) suggest that for T Tauri stars those centrally peaked line profiles are most likely a combination of emission from the inner part $(<\mathrm{a}$ few au) of a circumstellar disk and a slowly moving disk wind, launched by either EUV emission or soft X-rays. It is unclear whether a similar mechanism could explain our HAeBe observations given the weaker winds expended for these types of objects.

\subsection{CO compared to other gas tracers}

To get an as complete census of the gas around the studied HAeBes as possible, we complement the detected $\mathrm{CO}$ lines, $\mathrm{HI}$ recombination lines $\operatorname{Pf} \beta$ and $\mathrm{Hu} \epsilon$ and in one case the sodium doublet, with two other gas tracers. Here we focus on [O I] $6300 \AA$ and Polycyclic Aromatic Hydrocarbon (PAH) emission, which both trace the PP disk surface at different radial regimes. [OI] traces the gas up to the inner disk, between $\approx 0.1-50 \mathrm{au}$, and PAHs trace the outer disk, between $\approx 10$ and $100 \mathrm{au}$.

\subsection{1. $\mathrm{CO}$ and $[\mathrm{OI}]$ emission}

[O I] $6300 \AA$ A emission in HAeBe stars is the by-product of photodissociation of $\mathrm{OH}$ molecules. It traces those regions where FUV radiation impinges on the $\left(\mathrm{OH}, \mathrm{H}_{2} \mathrm{O}\right)$ gas in the atmosphere of circumstellar disks, and is commonly observed in $\mathrm{HAeBe}$ stars (Acke et al. 2005). Like fundamental CO emission, it traces the circumstellar disk from the innermost disk out to tens of au. We interpret this emission as a tracer of the disk atmosphere, and show a comparison between the $[\mathrm{O} \mathrm{I}]$ and $\mathrm{CO}$ emission lines in Fig. 9. With the exception of HD 101412, the [O I] lines are much broader than the CO lines, with a HW10M ratio that is typically 2.5 . We investigate this difference in line widths as a function of the HW10M radii in panel c of Fig. 8. The onset of the $[\mathrm{O} \mathrm{I}]$ emission is relatively constant at $0.8 \pm 0.4 \mathrm{au}$, whereas 


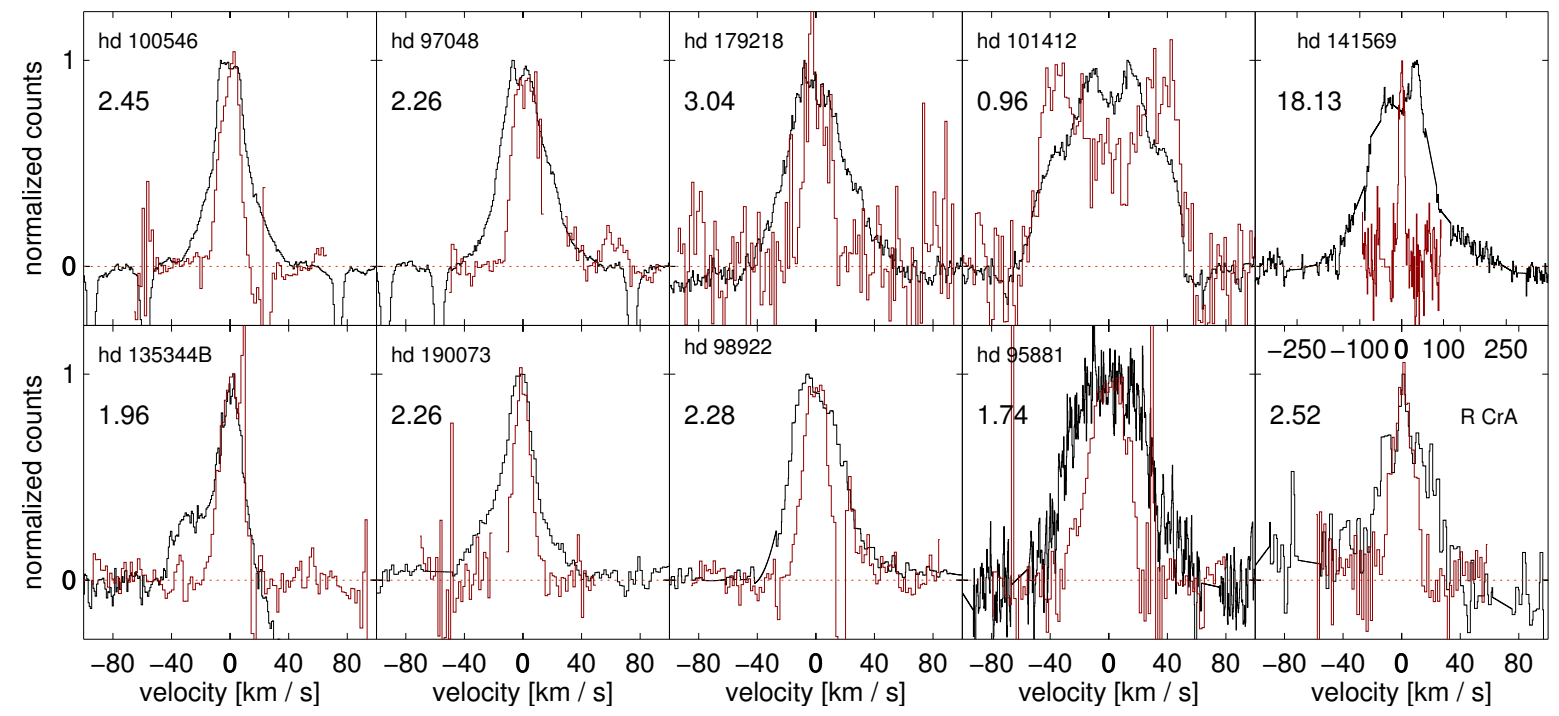

Fig. 9. $6300 \AA[\mathrm{OI}]$ emission (black histograms), and $\mathrm{CO} v=1-0 \mathrm{~J} \leq 15$ average line profiles (red lines), together with their $10 \%$ width [O I]/CO ratio. The CO emission of HD 141569 is averaged over the $v=2-1$ lines, and because of problems with the telluric correction, we have used the high $\left(J_{\mathrm{u}} \geq 30\right)$ for R CrA and HD 150193. The CO line profile plotted for HD 101412 is a blend of the ${ }^{13} \mathrm{CO} v=1-0 \mathrm{R}(23)$ and the ${ }^{12} \mathrm{CO} v=4-3 \mathrm{R}(35)$ lines. We show [OI] data of HD 97048 and HD 100546 from Acke \& van den Ancker (2006), of HD 101412, HD 135344B, and HD 179218 from van der Plas et al. (2008), of HD 190073 from van Lieshout and Bagnoli (priv. comm.; HERMES spectrograph at the Mercator telescope), and from Acke et al. (2005) for the other stars. We note that both the CO and [O I] lines have been centered at 0 velocity, and the differing velocity scale for HD 141569.

the onset of the $\mathrm{CO}$ emission changes from less than 1 to $10 \mathrm{au}$. The presence of this [OI] emission, together with the hydrogen recombination and sodium lines reported in Sect. 5.7, shows that the lack of $\mathrm{CO}$ emission at small radii does not imply a scarcity of gas closer to the star. This point is also illustrated by the direct comparison of $\mathrm{CO}$ and [O I] emission lines in Fig. 9, where, with the exception of HD 101412, the CO emission is about a factor of 2 narrower than the $[\mathrm{O} \mathrm{I}]$ line.

\subsection{2. $\mathrm{CO}$ and $\mathrm{PAH}$ emission}

PAHs are often detected and spatially resolved up to 100 au scales in the disks around HAeBe stars, and are a main contributor to the PP disk gas temperature in the upper layers of the disk due to the photo-electric heating of the gas (see e.g. Tielens 2008; Woitke et al. 2009). Meeus et al. (2001) first suggested a possible correlation between the strength of the PAH features and the shape of the IR SED, which was later confirmed by Acke \& van den Ancker (2004); Habart et al. (2004); Acke et al. (2010). Acke et al. (2010) find that the PAH-to-stellar luminosity ratio is higher in disks with a flared geometry, but that a few of their sources with a flattened dust disk still show relatively strong PAH emission. This picture was modified in a recent paper by Maaskant et al. (2014), who found that the PAH emission in transitional disks is dominated by the ionized PAH molecules residing in large disk gaps.

Because PAH molecules trace the gas disk, the presence of PAH emission in the IR spectra of HAeBe disks suggest that while the dust in these disks has already settled, the gas disk may still be flaring. This scenario of still flaring gas but settled dust has been previously suggested for two of our targets: HD 101412 (Sect. 7.3.3 and Fedele et al. 2008) and HD 95881 (Verhoeff et al. 2010). This scenario was also modeled and works if dust sedimentation is the dominant process in the disk. However, dust sedimentation does not explain the general trend of increasing PAH luminosity with increased flaring, but rather the opposite (Dullemond et al. 2007). We also place HD 98922 to the group of disks with settled dust but flaring gas based on the similarities in the dust (SED) and gas ([O I], PAH, CO ro-vibrational) diagnostics between HD 95881 and HD 98922.

We choose the $6.2 \mu \mathrm{m}$ PAH band as a proxy for the PAH emission because the relative luminosity of this feature displays the strongest correlation with far-IR excess. We use the PAH-to-stellar luminosity ratio to eliminate uncertainties in distance. We show the PAH-to-stellar luminosity ratio based on Spitzer IRS (Acke et al. 2010) and ISO-SWS (Acke \& van den Ancker 2004) spectra, in Table 5.

We plot the CO inner radius against the PAH-to-stellar luminosity ratio in panel $\mathrm{d}$ of Fig. 8. The self shadowed disks with no PAH detections have the smallest $\mathrm{CO}$ inner radii, and the inner $\mathrm{CO}$ radius increases with increasing $\mathrm{PAH}$ strength. The trend between disk-shape and PAH luminosity also holds for our sample, where the flaring disks show the strongest PAH emission, the PAHs in the self shadowed disks are not detected or have very low strength, and the disks with still flaring gas and selfshadowed dust show intermediate PAH emission.

\subsubsection{The special case of HD 101412}

HD 101412 is the only HAeBe star in our sample for which we detect both fundamental and first overtone $\mathrm{CO}$ emission, and where the $\mathrm{CO}$ line width is similar to the [O I] line width. The Spitzer-IRS spectrum of HD 101412 has an unusual shape due to the presence of strong PAH emission bands, and its SED is typical for a self-shadowed disk. However, it also displays some characteristics typical for a flaring disk, such as extended PAH emission (Fedele et al. 2008), and bright [O I] emission (Acke et al. 2005) suggesting that this disk might be transitioning from flaring to self-shadowed. Fedele et al. (2008) and van der Plas et al. (2008) have investigated the dust and gas components independently via high resolution spectroscopy of the [OI] $6300 \AA$ line and mid-IR interferometry (VLTI/MIDI). The $8-12 \mu \mathrm{m}$ emission has been resolved, and can be modeled by a ring between 0.4 and $1.9 \mathrm{au}$, and shows signs of asymmetry. 


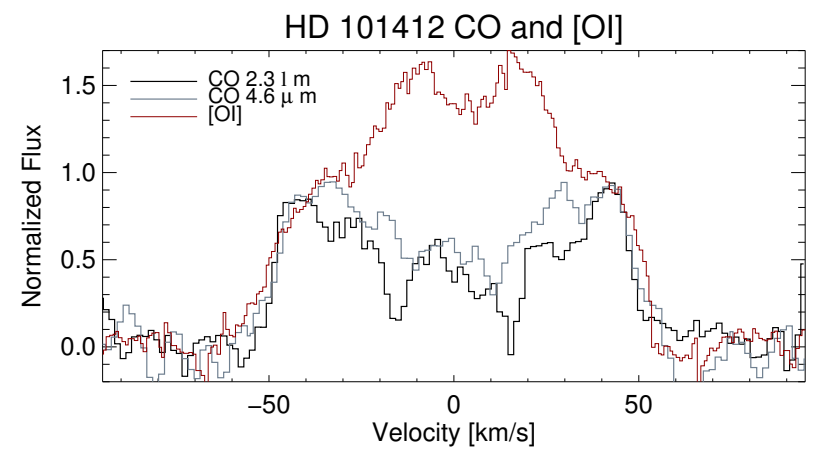

Fig. 10. Composite line profiles of $\mathrm{CO}$ fundamental (gray) and first overtone (black) emission in HD 101412, smoothed over 2 bins for clarity. Overplotted (red line) is the [O I] emission, scaled vertically for the high velocity components to overlap.

The PAH feature at $11.3 \mu \mathrm{m}$ is more extended than the continuum emission, and the [O I] emission shows 2 components: One originating from the inner rim, and the other between 6 and several tens of au. Both [O I] and PAH emission are thought to trace the part of the disk atmosphere that is directly exposed to the stellar UV field. Their conclusion is that the gas and dust in the disk around HD 101412 are decoupled, with a gas disk that rises out of the shadow cast by the inner rim after $\approx 6$ au.

Based on the kinematics of the fundamental and first overtone emission (both have the same radial velocity as the central star, the line widths of the fundamental and first overtone emission are similar, but the line center is more filled in for the fundamental emission), the $\mathrm{CO}$ emission is constrained to the inner disk region. We show the line profiles of [O I], fundamental and first overtone $\mathrm{CO}$ emission in Fig. 10. The comparable [O I] and CO line widths demonstrate that the onset of their emission in disks around HAeBe stars can be co-spatial. As discussed above, the dust and gas in the outer disk of HD 101412 are decoupled, and the [OI] emission originates from two components: a high projected velocity component from the hot inner rim, and a lower projected velocity component farther out. We test this interpretation to the first order by over plotting the (scaled) [O I] emission from HD 101412 in Fig. 10. The high projected velocity components of both the $[\mathrm{O} \mathrm{I}]$ and the $\mathrm{CO}$ emission are indeed co-spatial, but the low projected velocity component seen in the [O I] is not detected in the $\mathrm{CO}$ emission.

The detection of [O I] and non-detection of $\mathrm{CO}$ emission farther out in the disk demonstrates that the same trend as observed at small radii - $[\mathrm{O} \mathrm{I}]$ becomes, for whatever reason (dust settling, $\mathrm{CO}$ depletion, photo-destruction of $\mathrm{CO}$ ), visible sooner in the inner disk - also holds farther out in the disk. HD 101412 has a flaring gas ([O I], PAH) disk but settled dust disk. This suggests that the $\mathrm{CO}$ emission originates from deeper in the disk (closer to the dust, where it is either protected from dissociation and/or can be thermalized), and the [OI] emission - as by-product of photo-dissociation - traces those regions of the disk where the stellar UV flux dissociates the $\mathrm{OH}$ gas.

\subsection{Using CO ro-vibrational emission to find disk gaps}

It has recently been suggested by Maaskant et al. (2013) that all group I HAeBe stars have transitional (gapped) disks. The $\mathrm{CO}$ emission lines of all three group I sources in our sample show similar line shapes (narrow, flat topped) and dominant excitation condition (UV fluorescence). The disks around two of these objects show signs of harboring intermediate size gaps (HD $100546 \approx 13$ au, HD $97048 \approx 34 \pm 4$ au, Maaskant et al. 2013). CO emission from the inner wall of the outer disk, which is in full exposure of the stellar UV flux, would be a natural way to explain the narrow line profiles and the UV fluoresced $\mathrm{CO}$ molecules. Based on this similarity, we suggest that the disk around the 3rd flaring disk, HD 179218, harbors a similar gap. Given the larger distance to this source a gap similar to the gap in the disk around HD 100546, and possibly HD 97048, may well have been missed in modeling efforts based on the SED alone.

Not all HAeBe disks with a disk gap show CO emission properties similar to the 3 group I sources discussed in this paper. The $\mathrm{CO}$ emission from $\mathrm{AB}$ Aur for example is dominated by the inner disk (Brittain et al. 2003; Blake \& Boogert 2004), which extends out to $\approx 40$ au. The UV field at the inner rim of the outer disk at $\approx 300$ au is likely too weak to play a significant role in exciting to $\mathrm{CO}$ molecules, and the gas at that location is likely so cold that the ro-vibrational $\mathrm{CO}$ emissivity is negligible. A similar case can be made for the disk around HD 135344B.

We suggest that the three group I sources in our sample trace a specific kind of transitional disks: those with a hole or an inner disk that is small enough so that its $\mathrm{CO}$ emission does not dominate the emission spectrum, and with an outer disk that is close enough to the star to be both warm enough to thermally excite the $\mathrm{CO}$ molecules and in contact with a strong enough (stellar) UV field to fluoresce the CO molecules.

\section{Conclusions}

In this paper we present detections of ro-vibrational fundamental $\mathrm{CO}$ emission in 12 out of 13 surveyed HAeBe stars. We investigate the kinematics and temperature of this gas, and correlate these with disk dust properties (the amount of disk flaring), and other disk-gas tracers: PAH and [O I] emission. Keeping in mind the modest sample size, we report the following trends between targets, and within the different isotopologues and vibrational bands for each target:

[1.] CO fundamental emission is common in disks around HAeBe stars;

[2.] $\mathrm{CO}$ first overtone emission is only detected in one out of 13 surveyed disks. This is consistent with the $7 \%$ detection rate of $\mathrm{CO}$ overtone emission from $\mathrm{HAeBe}$ disks reported by Ilee et al. (2014);

[3.] The $\mathrm{CO}$ emission in the group I sources originates from larger distances than the $\mathrm{CO}$ in group II disks;

[4.] The $\mathrm{CO}$ emission in $\mathrm{R} \mathrm{CrA}$ is most likely originating from an outfow rather than from a disk;

[5.] The rotational temperature of the ${ }^{13} \mathrm{CO}$ is lower than that of the ${ }^{12} \mathrm{CO}$;

[6.] We detect a broadening of the emission lines as a function of excitation temperature in the disks around two group II sources: HD 98922 and HD 135344B. Most other group II sources are difficult to classify due to low $\mathrm{S} / \mathrm{N}$. The CO emission in the higher vibrational bands of the group I disks, however, does not show this trend;

[7.] The $4.6 \mu \mathrm{m}$ dust continuum emission in all three group I disks, and the CO emission in two group I disks is spatially resolved;

[8.] The dominant excitation mechanism for the $\mathrm{CO}$ vibrational band populations is thermal or pumping by IR radiation for group II sources, and fluorescence for the group I sources; 
[9.] We propose that the presence of fluoresced $\mathrm{CO}$ in Herbig $\mathrm{Ae} / \mathrm{Be}$ disks is a proxy for the presence of moderately sized gaps in their disks;

[10.] Following this recommendation, we suggest that the disk around HD 179218 is a (pre) transitional disk (harbors a disk gap or hole) based on the $\mathrm{CO}$ line width and excitation mechanism.

These findings are consistent with a picture in which the $\mathrm{CO}$ emission is dominated by the disk surface. In the group I disks the $\mathrm{CO}$ emitting surface extends up to large distances, but starts at radii larger than the dust sublimation radius, whereas in the group II disks the $\mathrm{CO}$ emanates in a narrow region closer to the dust sublimation radius. There is a strong correlation between the dominant mode of CO excitation and the topology of the circumstellar dust disk. The group I sources have line shapes that do not correlate with excitation energy; their higher vibrational bands are over populated compared to the expected values belonging to the rotational temperature of the gas, and their dust continuum and sometimes $\mathrm{CO}$ emission are spatially resolved up to tens of au. The self-shadowed dust disks (group II sources) in contrast do not show these signs.

The difference between the observed $\mathrm{CO}$ emission properties of group I and group II sources are a direct reflection of differences in the excitation mechanism of the gas in the $\mathrm{CO}$ emitting region in both groups. This difference appears to be driven by a difference in radial location of the emitting region. Whereas in group II sources the $\mathrm{CO}$ emitting region is confined to the inner dust disk, in the group I sources the $\mathrm{CO}$ emitting region is more extended, and significant amounts of the observed $\mathrm{CO}$ emission originate from radii up to tens of au. The above picture is reminiscent of the interpretation by Maaskant et al. (2013, 2014) for the presence of ionized PAH emission and spatially resolved $Q$-band emission in group I disks, where they argue that disk gaps are necessary to explain the observed phenomenology. In this picture, the fluorescent $\mathrm{CO}$ emission we have observed in the group I sources in our sample originates from the inner rim of the outer disk (in the cases where this rim is warm enough to thermally excite the rotational $\mathrm{CO}$ ladder). We thus suggest that the presence of fluoresced $\mathrm{CO}$ in Herbig Ae/Be disks is a proxy for the presence of moderately sized gaps in their disks.

Acknowledgements. G.v.d.P. offers his gratitude to B. Acke, R. van Lieshout and T. Bagnoli for providing the [OI] spectra used in Fig. 9; to the Paranal staff for their assistance during the observations; to I. Kamp, A. Carmona, R. H. Bertelsen, W. F. Thi and the anonymous referee fotheir comments and discussions that have helped strengthen this work. G.v.d.p. acknowledges support from the Millennium Science Initiative (Chilean Ministry of Economy) through grant Nucleus P10-022-F and acknowledges financial support provided by FONDECYT following grant 3140393. This publication makes use of data products from the Two Micron All Sky Survey, which is a joint project of the University of Massachusetts and the Infrared Processing and Analysis Center/California Institute of Technology, funded by the National Aeronautics and Space Administration and the National Science Foundation.

\section{References}

Acke, B., \& van den Ancker, M. E. 2004, A\&A, 426, 151

Acke, B., \& van den Ancker, M. E. 2006, A\&A, 449, 267

Acke, B., van den Ancker, M. E., \& Dullemond, C. P. 2005, A\&A, 436, 209

Acke, B., Bouwman, J., Juhász, A., et al. 2010, ApJ, 718, 558

Alecian, E., Wade, G. A., Catala, C., et al. 2013, MNRAS, 429, 1001

Alexander, R., Pascucci, I., Andrews, S., Armitage, P., \& Cieza, L. 2014, in

Protostars \& Planets VI (University of Arizona press), in press

Andrae, R., Schulze-Hartung, T., \& Melchior, P. 2010 [arXiv: 1012 . 3754]

Ardila, D. R., Golimowski, D. A., Krist, J. E., et al. 2007, ApJ, 665, 512

Baines, D., Oudmaijer, R. D., Porter, J. M., \& Pozzo, M. 2006, MNRAS, 367, 737
Bast, J. E., Brown, J. M., Herczeg, G. J., van Dishoeck, E. F., \& Pontoppidan, K. M. 2011, A\&A, 527, A119

Beckwith, S. V. W., \& Sargent, A. I. 1993, ApJ, 402, 280

Benisty, M., Tatulli, E., Ménard, F., \& Swain, M. R. 2010, A\&A, 511, A75

Berthoud, M. G. 2008, Ph.D. Thesis

Blake, G. A., \& Boogert, A. C. A. 2004, ApJ, 606, L73

Blondel, P. F. C., \& Tjin A Djie, H. R. E. 2006, A\&A, 456, 1045

Brittain, S. D., Rettig, T. W., Simon, T., et al. 2003, ApJ, 588, 535

Brittain, S. D., Simon, T., Najita, J. R., \& Rettig, T. W. 2007, ApJ, 659, 685

Brittain, S. D., Najita, J. R., \& Carr, J. S. 2009, ApJ, 702, 85

Brown, J. M., Blake, G. A., Dullemond, C. P., et al. 2007, ApJ, 664, L107

Brown, J. M., Pontoppidan, K. M., van Dishoeck, E. F., et al. 2013, ApJ, 770, 94

Calvet, N., Muzerolle, J., Briceño, C., et al. 2004, AJ, 128, 1294

Carmona, A. 2010, Earth Moon and Planets, 106, 71

Carmona, A., van den Ancker, M. E., Thi, W.-F., Goto, M., \& Henning, T. 2005, A\&A, 436, 977

Carmona, A., Pinte, C., Thi, W. F., et al. 2014, A\&A, 567, A51

Carr, J. S. 1989, ApJ, 345, 522

Carr, J. S., Tokunaga, A. T., Najita, J., Shu, F. H., \& Glassgold, A. E. 1993, ApJ, 411, L37

Catala, C., Alecian, E., Donati, J.-F., et al. 2007, A\&A, 462, 293

Chandra, S., Maheshwari, V. U., \& Sharma, A. K. 1996, A\&AS, 117, 557

Choi, M., Hamaguchi, K., Lee, J.-E., \& Tatematsu, K. 2008, ApJ, 687, 406

Clark, J. S., Steele, I. A., Fender, R. P., \& Coe, M. J. 1999, A\&A, 348, 888

Correia, S., Köhler, R., Meeus, G., \& Zinnecker, H. 2008, The Power of

Optical/IR Interferometry: Recent Scientific Results and 2nd Generation, 175

Cowley, C. R., Hubrig, S., Castelli, F., \& Wolff, B. 2012, A\&A, 537, L6

de Zeeuw, P. T., Hoogerwerf, R., de Bruijne, J. H. J., Brown, A. G. A., \& Blaauw,

A. 1999, AJ, 117, 354

Dent, W. R. F., Greaves, J. S., \& Coulson, I. M. 2005, MNRAS, 359, 663

de Winter, D., van den Ancker, M. E., Maira, A., et al. 2001, A\&A, 380, 609

Dullemond, C. P., Dominik, C., \& Natta, A. 2001, ApJ, 560, 957

Dullemond, C. P., Henning, T., Visser, R., et al. 2007, A\&A, 473, 457

Dunkin, S. K., Barlow, M. J., \& Ryan, S. G. 1997, MNRAS, 290, 165

Eisner, J. A., Lane, B. F., Hillenbrand, L. A., Akeson, R. L., \& Sargent, A. I. 2004, ApJ, 613, 1049

Eisner, J. A., Graham, J. R., Akeson, R. L., \& Najita, J. 2009, ApJ, 692, 309

Fedele, D., van den Ancker, M. E., Acke, B., et al.e 2008, A\&A, 491, 809

Forbrich, J., Preibisch, T., \& Menten, K. M. 2006, A\&A, 446, 155

Fukagawa, M., Tamura, M., Itoh, Y., Hayashi, S. S., \& Oasa, Y. 2003, ApJ, 590, L49

Garcia Lopez, R., Natta, A., Testi, L., \& Habart, E., 2006, A\&A, 459, 837

Grady, C. A., Woodgate, B., Torres, C. A. O., et al. 2004, ApJ, 608, 809

Grady, C. A., Woodgate, B., Heap, S. R., et al. 2005, ApJ, 620, 470

Goldsmith, P. F., \& Langer, W. D. 1999, ApJ, 517, 209

Goto, M., Usuda, T., Takato, N., et al. 2003, ApJ, 598, 1038

Goto, M., Usuda, T., Dullemond, C. P., et al. 2006, ApJ, 652, 758

Goto, M., van der Plas, G., van den Ancker, M., et al. 2012, A\&A, 539, A81

Habart, E., Natta, A., \& Krügel, E. 2004, A\&A, 427, 179

Hein Bertelsen, R. P., Kamp, I., Goto, M., et al. 2014, A\&A, 561, A102

Hillenbrand, L. A., Strom, S. E., Vrba, F. J., \& Keene, J. 1992, ApJ, 397, 613

Hughes, A. M., Wilner, D. J., Andrews, S. M., Qi, C., \& Hogerheijde, M. R. 2011, ApJ, 727, 85

Ilee, J. D., Fairlamb, J., Oudmaijer, R. D., et al. 2014, MNRAS, 445, 3723

Käufl, H.-U., Ballester, P., Biereichel, P., et al. 2004, Proc. SPIE, 5492, 1218

Kilkenny, D., Whittet, D. C. B., Davies, J. K., et al. 1985, South African Astronomical Observatory Circular, 9, 55

Knude, J., \& Hog, E. 1998, A\&A, 338, 897

Köhler, R., Neuhäuser, R., Krämer, S., et al. 2008, A\&A, 488, 997

Kraus, S., Hofmann, K.-H., Benisty, M., et al. 2008, A\&A, 489, 1157

Kraus, S., Hofmann, K.-H., Malbet, F., et al. 2009, A\&A, 508, 787

Krotkov, R., Wang, D., \& Scoville, N. Z. 1980, ApJ, 240, 940

Kumar, M. S. N., Sharma, S., Davis, C. J., Borissova, J., \& Grave, J. M. C. 2011, A\&A, 533, A137

Kurucz, R. L. 1991, Precision Photometry: Astrophysics of the Galaxy, eds. A. G. D. Philip, A. R. Upgren, \& K. A. Janes, 27

Lagage, P.-O., Doucet, C., Pantin, E., et al. 2006, Science, 314, 621

Langer, W. D., \& Penzias, A. A. 1990, ApJ, 357, 477

Liu, W. M., Hinz, P. M., Meyer, M. R., et al. 2007, ApJ, 658, 1164

Lorenzetti, D.,Larionov, V. M., Giannini, T., et al. 2009, ApJ, 693, 1056

Maaskant, K. M., Honda, M., Waters, L. B. F. M., et al. 2013, A\&A, 555, A64

Maaskant, K. M., Min, M., Waters, L. B. F. M., \& Tielens, A. G. G. M. 2014, A\&A, 563, A78

Mannings, V., \& Sargent, A. I. 1997, ApJ, 490, 792

Mannings, V., \& Sargent, A. I. 2000, ApJ, 529, 391

Marraco, H. G., \& Rydgren, A. E. 1981, AJ, 86, 62

Marsh, K. A., Silverstone, M. D., Becklin, E. E., et al. 2002, ApJ, 573, 425

McGregor, P. J., Hyland, A. R., \& Hillier, D. J. 1988, ApJ, 324, 1071 
Meeus, G., Waelkens, C., \& Malfait, K. 1998, A\&A, 329, 131

Meeus, G., Waters, L. B. F. M., Bouwman, J., et al. 2001, A\&A, 365, 476

Mendigutía, I., Calvet, N. Montesinos, B., et al. 2011, A\&A, 535, A99

Merín, B., Montesinos, B., Eiroa, C., et al. 2004, A\&A, 419, 301

Mitchell, G. F., Maillard, J.-P., Allen, M., Beer, R., \& Belcourt, K. 1990, ApJ, 363,554

Monnier, J. D., \& Millan-Gabet, R. 2002, ApJ, 579, 694

Monnier, J. D., Berger, J.-P., Millan-Gabet, R., et al. 2006, ApJ, 647, 444

Mulders, G. D., Min, M., Dominik, C., Debes, J. H., \& Schneider, G. 2013, A\&A, 549, A112

Najita, J., Carr, J. S., Glassgold, A. E., Shu, F. H., \& Tokunaga, A. T. 1996, ApJ, 462,919

Najita, J., Carr, J. S., \& Mathieu, R. D. 2003, ApJ, 589, 931

Oudmaijer, R. D., Proga, D., Drew, J. E., \& de Winter, D. 1998, MNRAS, 300, 170

Panić, O., \& Hogerheijde, M. R. 2009, A\&A, 508, 707

Pontoppidan, K. M., Blake, G. A., van Dishoeck, E. F., et al. 2008, ApJ, 684, 1323

Pontoppidan, K. M., Blake, G. A., \& Smette, A. 2011, ApJ, 733, 84

Reipurth, B., Pedrosa, A., \& Lago, M. T. V. T. 1996, A\&AS, 120, 229

Rosenfeld, K. A., Andrews, S. M., Hughes, A. M., Wilner, D. J., \& Qi, C. 2013, ApJ, 774, 16

Rothman, L. S., et al. 1992, J. Quant. Spectr. Rad. Trans., 48, 469

Salyk, C., Blake, G. A., Boogert, A. C. A., \& Brown, J. M. 2009, ApJ, 699, 330

Salyk, C., Herczeg, G. J., Brown, J. M., et al. 2013, ApJ, 769, 21

Scoville, N. Z., Krotkov, R., \& Wang, D. 1980, ApJ, 240, 929
Scoville, N., Kleinmann, S. G., Hall, D. N. B., \& Ridgway, S. T. 1983, ApJ, 275, 201

Skrutskie, M. F., Cutri, R. M., Stiening, R., et al. 2006, AJ, 131, 1163

Tatulli, E., Benisty, M., Ménard, F., et al. 2011, A\&A, 531, A1

The, P. S., de Winter, D., \& Perez, M. R. 1994, A\&AS, 104, 315

Thi, W.-F., van Dalen, B., Bik, A., \& Waters, L. B. F. M. 2005, A\&A, 430, L61

Thi, W. F., Kamp, I., Woitke, P., et al. 2013, A\&A, 551, A49

Thompson, R. I., \& Boroson, T. A. 1977, ApJ, 216, L75

Tielens, A. G. G. M. 2008, ARA\&A, 46, 289

van Boekel, R., Min, M., Waters, L. B. F. M., et al. 2005, A\&A, 437, 189

van den Ancker, M. 2005, in ESO Symp.: High Resolution Infrared Spectroscopy in Astronomy (Springer), 309

van den Ancker, M. E., de Winter, D., \& Tjin A Djie, H. R. E. 1998, A\&A, 330, 145

van den Ancker, M. E., Meeus, G., Cami, J., Waters, L. B. F. M., \& Waelkens, C. 2001, A\&A, 369, L17

Van Der Plas, G. 2010, Ph.D. Thesis

van der Plas, G., van den Ancker, M. E., Fedele, D., et al. 2008, A\&A, 485, 487 van der Plas, G., van den Ancker, M. E., Acke, B., et al. 2009, A\&A, 500, 1137

Verhoeff, A. P., Min, M., Acke, B., et al. 2010, A\&A, 516, A48

Sylvester, R. J., Skinner, C. J., Barlow, M. J., \& Mannings, V. 1996, MNRAS, 279,915

Weinberger, A. J., Becklin, E. E., Schneider, G., et al. 1999, ApJ, 525, L53

Weintraub, D. A., Zuckerman, B., \& Masson, C. R. 1989, ApJ, 344, 915

Woitke, P., Kamp, I., \& Thi, W.-F. 2009, A\&A, 501, 383 
G. van der Plas et al.: CO ro-vibrational line survey of HAeBe disks

\section{Appendix A: Confidence intervals for $T_{\mathrm{ex}}$ and $N(\mathrm{CO})$ determination}

To compare our observations with the emission expected from an isothermal slab model with a given $T_{\mathrm{ex}}$ and $N(\mathrm{CO})$, we minimize the sum over all detections within one ro-vibrational transition following $\frac{1}{n_{\mathrm{obs}}} \frac{\text { (model-observation) }^{2}}{\text { error }^{2}}$ in the parameter space between
$100 \mathrm{~K} \leq T_{\mathrm{ex}} \leq 2500 \mathrm{~K}$ and $10^{16} \mathrm{~cm}^{-2} \leq N(\mathrm{CO}) \leq 10^{20} \mathrm{~cm}^{-2}$ for ${ }^{12} \mathrm{CO}$ emission (Fig. A.1), and $100 \mathrm{~K} \leq T_{\mathrm{ex}} \leq 1200 \mathrm{~K}$ and $10^{17} \mathrm{~cm}^{-2} \leq N(\mathrm{CO}) \leq 10^{21} \mathrm{~cm}^{-2}$ for ${ }^{13} \mathrm{CO}$ emission (Fig. A.2), in steps of $\Delta T_{\mathrm{ex}}=25 \mathrm{~K}$ and $\Delta^{10} \log (N(\mathrm{CO}))=0.1$. We define our fitting error as to all solutions within a $1 \sigma$ confidence interval of the best fit value. The best fit values are listed in Table 3.
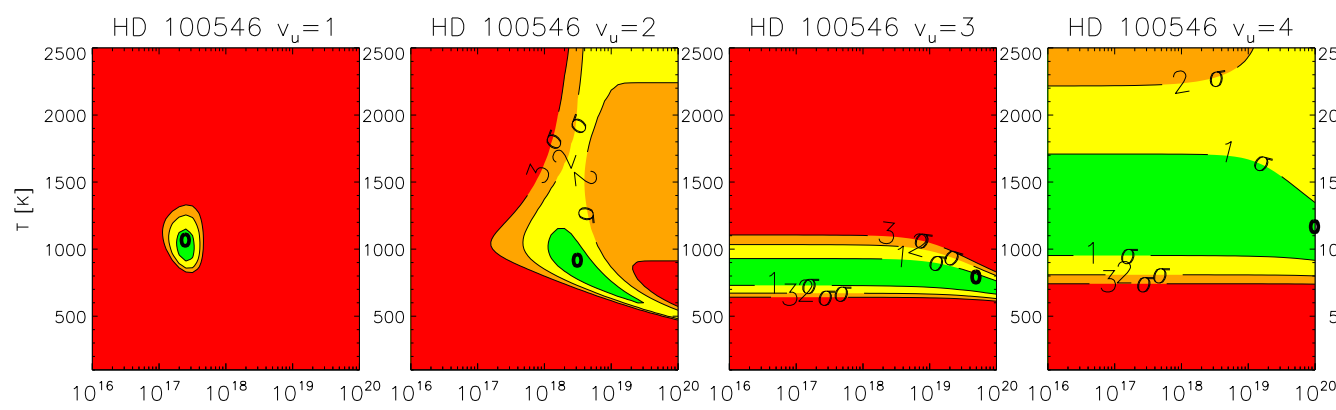

HD $100546 v_{u}=5$

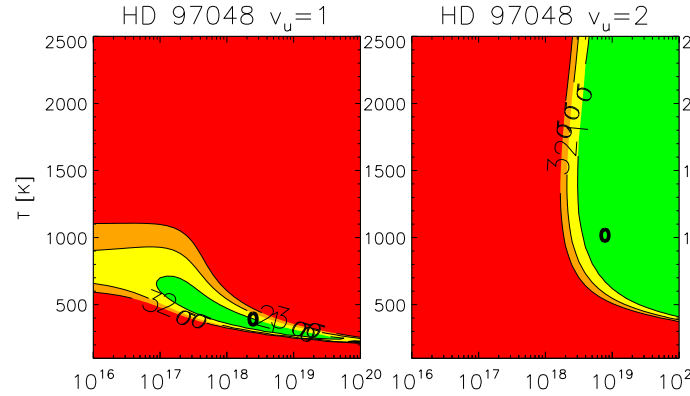

HD $97048 v_{u}=3$
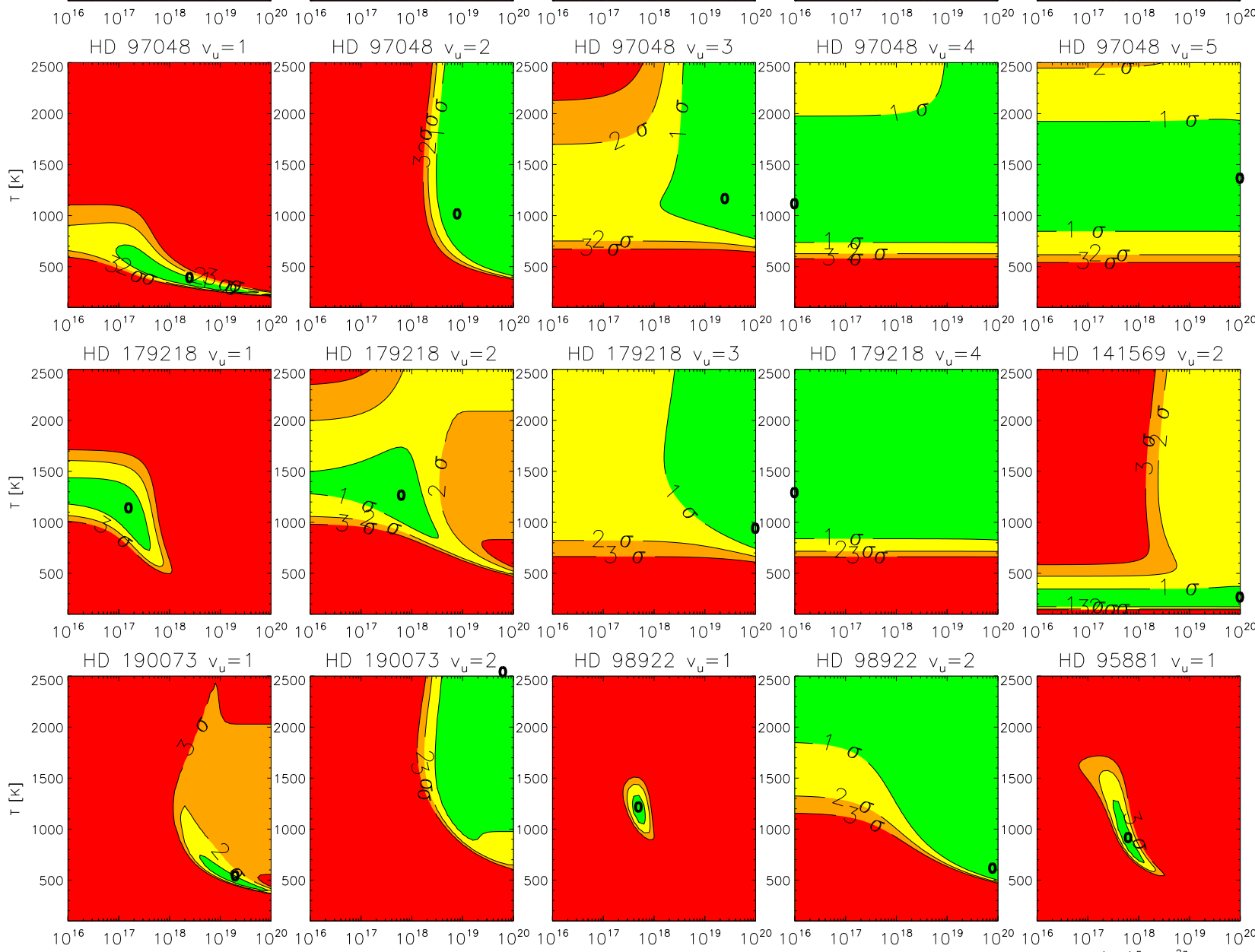

HD $98922 v_{u}=1$
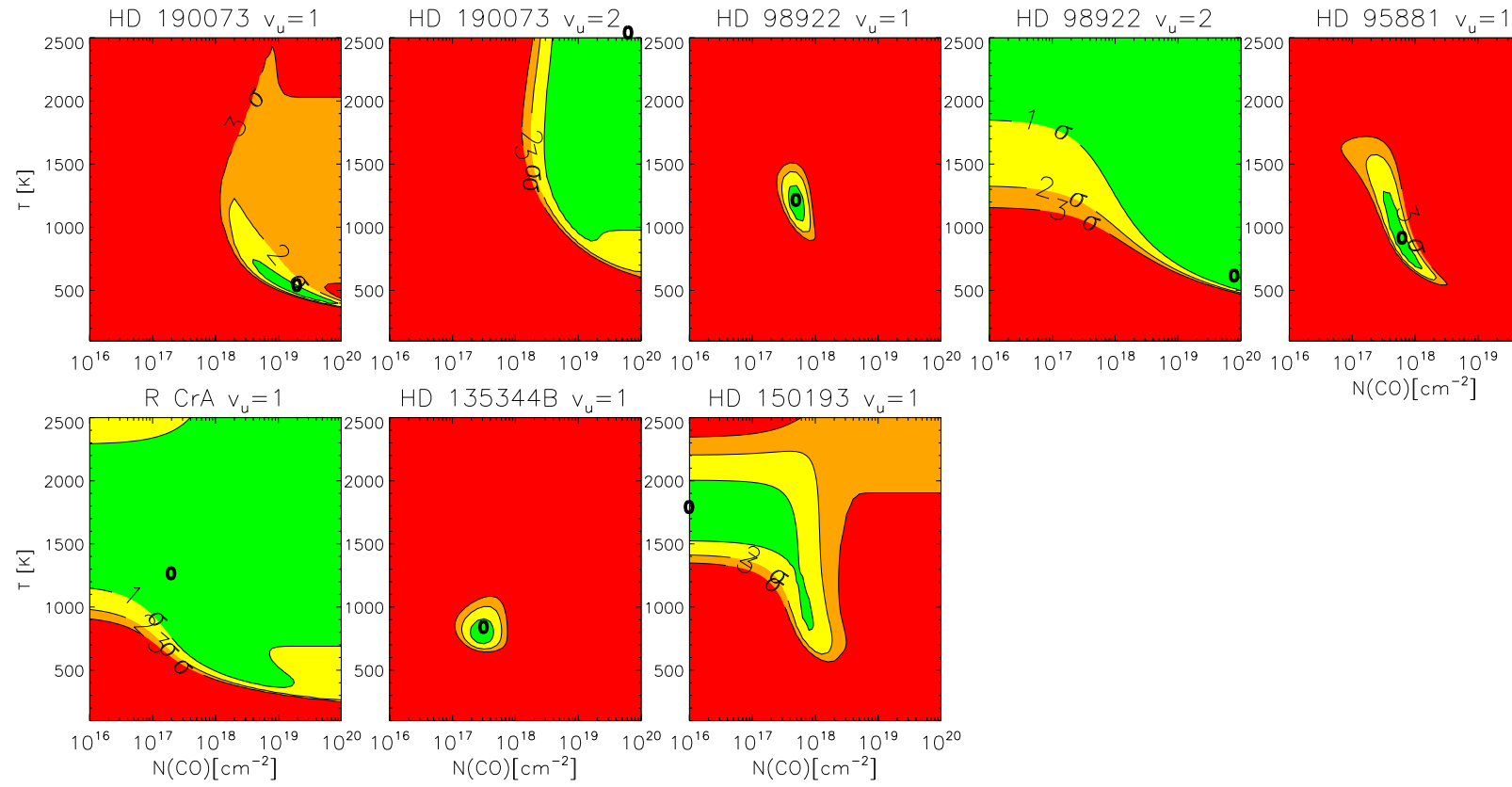

Fig. A.1. Best fit contours for associated ${ }^{12} \mathrm{CO}$ Boltzmann plots. Color contours denote deviations of $1 \sigma$ (green), $2 \sigma$ (yellow), $3 \sigma$ (orange) and $>3 \sigma$ (red) confidence intervals. 

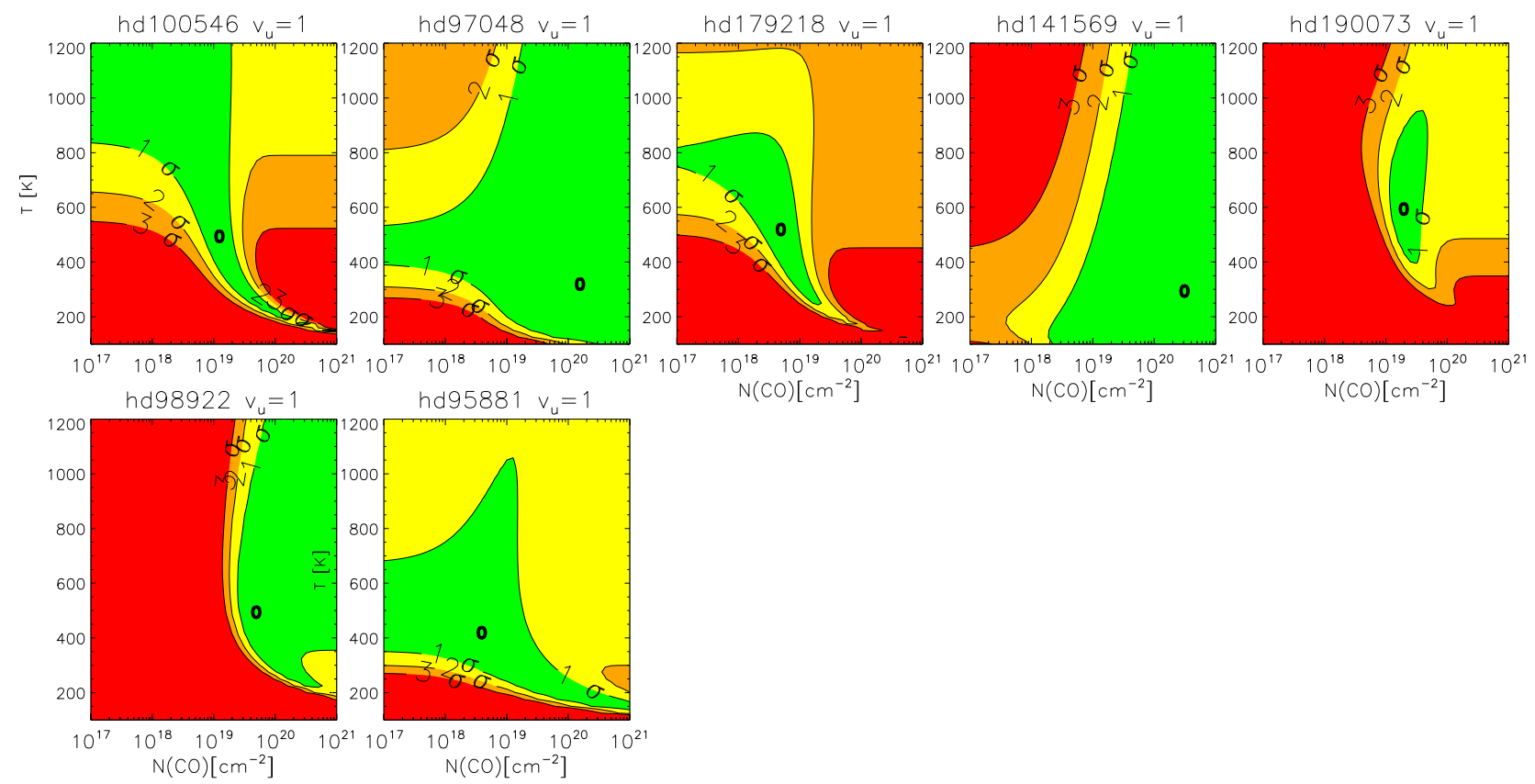

Fig. A.2. Best fit contours for associated ${ }^{13} \mathrm{CO}$ Boltzmann plots. Color contours denote deviations of $1 \sigma$ (green), $2 \sigma$ (yellow), $3 \sigma$ (orange) and $>3 \sigma$ (red) confidence intervals.

\section{Appendix B: Line flux tables}

We list the fluxes and upper limits of the $\mathrm{CO} v=2-0 \mathrm{R} 03$ lines in Table B. 2 and those of the Pfund $\beta$ hydrogen recombination line in Table B.3. We were unable to identify this line in the spectrum of HD 101412 due to the many strong and overlapping ro-vibrational CO lines in the line region (c.g. Fig. 1).

We flux-calibrate the $\mathrm{CO}$ overtone spectra using the $K$ s magnitudes at $2.17 \mu \mathrm{m}$ as published in the 2 MASS catalog (Skrutskie et al. 2006), and the Pfund $\beta$ and CO fundamental spectra with the $4.77 \mu$ m flux, as determined with a spline interpolation to the $M$ band data points, from (e.g. Kilkenny et al. 1985; Hillenbrand et al. 1992; de Winter et al. 2001).
Table B.1. Continuum flux values used for calculating the line fluxes listed in Tables B.2-B.4.

\begin{tabular}{lll}
\hline \hline Target & $\begin{array}{l}F_{\text {continuum, } 2.17 \mu \mathrm{m}} \\
{\left[\mathrm{erg} \mathrm{cm}{ }^{-2} \mu \mathrm{m}^{-1} \mathrm{~s}^{-1}\right]}\end{array}$ & $\begin{array}{l}F_{\text {continuum,4.77 } \mu \mathrm{m}} \\
{\left[\mathrm{erg} \mathrm{cm}^{-2} \mu \mathrm{m}^{-1} \mathrm{~s}^{-1}\right]}\end{array}$ \\
\hline HD 100546 & $2.8 \times 10^{-9}$ & $6.9 \times 10^{-10}$ \\
HD 97048 & $2.0 \times 10^{-9}$ & $3.1 \times 10^{-10}$ \\
HD 179218 & $3.1 \times 10^{-8}$ & $4.7 \times 10^{-10}$ \\
HD 101412 & $2.2 \times 10^{-9}$ & $2.0 \times 10^{-10}$ \\
HD 141569 & $2.0 \times 10^{-9}$ & $5.5 \times 10^{-11}$ \\
HD 190073 & $8.0 \times 10^{-10}$ & $5.8 \times 10^{-10}$ \\
HD 98922 & & $2.7 \times 10^{-9}$ \\
R CrA & $1.7 \times 10^{-9}$ & $8.1 \times 10^{-9}$ \\
HD 135344B & $1.8 \times 10^{-9}$ & $3.6 \times 10^{-10}$ \\
HD 150193 & $2.9 \times 10^{-9}$ & $6.9 \times 10^{-10}$ \\
HD 104237 & & $1.1 \times 10^{-9}$ \\
HD 95881 & $4.4 \times 10^{-10}$ & $6.6 \times 10^{-10}$ \\
\hline
\end{tabular}


G. van der Plas et al.: CO ro-vibrational line survey of HAeBe disks

Table B.2. Line fluxes and upper limits of the CO $v=2-0 \mathrm{R} 03$ line at $2337.4128 \mathrm{~nm}$.

\begin{tabular}{lll}
\hline \hline Target & Line ID & $\begin{array}{l}F_{\text {line }} \\
{\left[\mathrm{erg} \mathrm{cm}^{-2} \mathrm{~s}^{-1}\right]}\end{array}$ \\
\hline HD 100546 & ${ }^{12} \mathrm{CO} v=2-0 \mathrm{R} 03$ & $<8.1 \times 10^{-15}$ \\
HD 97048 & ${ }^{12} \mathrm{CO} v=2-0 \mathrm{R} 03$ & $<1.2 \times 10^{-14}$ \\
HD 179218 & ${ }^{12} \mathrm{CO} v=2-0$ R03 & $<1.2 \times 10^{-13}$ \\
HD 101412 & ${ }^{12} \mathrm{CO} v=2-0$ R03 & $4.6 \times 10^{-14}$ \\
HD 141569 & ${ }^{12} \mathrm{CO} v=2-0$ R03 & $<1.04 \times 10^{-14}$ \\
HD 190073 & ${ }^{12} \mathrm{CO} v=2-0$ R03 & $<5.5 \times 10^{-15}$ \\
HD 95881 & ${ }^{12} \mathrm{CO} v=2-0$ R03 & $<2.2 \times 10^{-15}$ \\
R CrA & ${ }^{12} \mathrm{CO} v=2-0$ R03 & $-1.0 \times 10^{-14}$ \\
HD 135344 & ${ }^{12} \mathrm{CO} v=2-0$ R03 & $<8.9 \times 10^{-15}$ \\
HD 150193 & ${ }^{12} \mathrm{CO} v=2-0$ R03 & $<1.3 \times 10^{-14}$ \\
\hline
\end{tabular}

Notes. Detection limits are $3 \sigma$ assuming a line width of $20 \mathrm{~km} \mathrm{~s}^{-1}$ and the continuum fluxes listed in Table B.1. We note that there are no CO overtone observations for HD 98922, HD 104237 and HD 142666.

Table B.3. Pfund $\beta$ line fluxes and FWHM values for the programme stars.

\begin{tabular}{lllll}
\hline \hline Target & $\begin{array}{l}F W H M \\
{\left[\mathrm{~km} \mathrm{~s}^{-1}\right]}\end{array}$ & $\begin{array}{l}\text { Error } \\
{\left[\mathrm{km} \mathrm{s}^{-1}\right]}\end{array}$ & $\begin{array}{l}\text { Line flux } \\
{\left[\mathrm{erg} \mathrm{cm}^{-2} \mathrm{~s}^{-1}\right]}\end{array}$ & $\begin{array}{l}\text { Error } \\
{\left[\mathrm{erg} \mathrm{cm}^{-2} \mathrm{~s}^{-1}\right]}\end{array}$ \\
\hline HD 100546 & 218 & 17 & $8.75 \times 10^{-13}$ & $1.31 \times 10^{-13}$ \\
HD 97048 & 149 & 10 & $5.71 \times 10^{-13}$ & $9.99 \times 10^{-14}$ \\
HD 179218 & 150 & 10 & $8.19 \times 10^{-13}$ & $9.35 \times 10^{-14}$ \\
HD 101412 & - & - & $<8.7 \times 10^{-14}$ & \\
HD 141569 & 420 & 60 & $1.19 \times 10^{-13}$ & $3.32 \times 10^{-14}$ \\
HD 190073 & 92 & 9 & $3.41 \times 10^{-13}$ & $8.68 \times 10^{-14}$ \\
HD 98922 & 140 & 12 & $1.07 \times 10^{-12}$ & $2.67 \times 10^{-13}$ \\
HD 95881^ & - & - & - & - \\
R CrA & 230 & 40 & $2.82 \times 10^{-12}$ & $6.45 \times 10^{-13}$ \\
HD 135344B & 142 & 10 & $8.06 \times 10^{-14}$ & $4.03 \times 10^{-15}$ \\
HD 150193* & - & - & - & - \\
HD 104237 & 117 & 14 & $1.08 \times 10^{-12}$ & $1.14 \times 10^{-13}$ \\
HD 142666 & 325 & 30 & $5.82 \times 10^{-14}$ & $1.77 \times 10^{-13}$ \\
\hline
\end{tabular}

Notes. Line fluxes are calculated using the continuum fluxes listed in Table B.1. We note that for HD 95881 and HD 150193 (marked with a " $\star$ "), the Pfund $\beta$ line there are lines visible in the raw spectra and have a respective FWHM of $\approx 100$ and $180 \mathrm{~km} \mathrm{~s}^{-1}$ and a maximum intensity of $\approx 20 \%$ and $15 \%$ above the continuum. Because of the presence of Pfund $\beta$ emission in the spectrum of the telluric standard star we are unable to calculate the line fluxes, and therefore do not list these values in the table.

Table B.4. Line flux and error values used in this manuscript.

\begin{tabular}{llll}
\hline \hline Target & Line id & $\begin{array}{l}\text { Line flux } \\
{\left[\mathrm{erg} \mathrm{cm}^{-2} \mathrm{~s}^{-1}\right]}\end{array}$ & $\begin{array}{l}\text { Error } \\
{\left[\mathrm{erg} \mathrm{cm}^{-2} \mathrm{~s}^{-1}\right]}\end{array}$ \\
\hline HD 100546 & ${ }^{12} \mathrm{CO} v(1-0) \mathrm{R} 08$ & $1.18 \times 10^{-13}$ & $4.94 \times 10^{-15}$ \\
& ${ }^{12} \mathrm{CO} v(1-0) \mathrm{R} 07$ & $1.18 \times 10^{-13}$ & $4.70 \times 10^{-15}$ \\
& ${ }^{12} \mathrm{CO} v(1-0)$ R06 & $1.30 \times 10^{-13}$ & $5.11 \times 10^{-15}$ \\
& ${ }^{12} \mathrm{CO} v(1-0) \mathrm{R} 06$ & $1.22 \times 10^{-13}$ & $5.01 \times 10^{-15}$ \\
& ${ }^{12} \mathrm{CO} v(1-0) \mathrm{R} 03$ & $1.29 \times 10^{-13}$ & $4.27 \times 10^{-15}$ \\
& ${ }^{12} \mathrm{CO} v(1-0) \mathrm{R} 02$ & $9.54 \times 10^{-14}$ & $3.48 \times 10^{-15}$ \\
& ${ }^{12} \mathrm{CO} v(1-0) \mathrm{R} 01$ & $7.50 \times 10^{-14}$ & $3.19 \times 10^{-15}$ \\
& ${ }^{12} \mathrm{CO} v(1-0) \mathrm{P} 02$ & $5.55 \times 10^{-14}$ & $3.00 \times 10^{-15}$ \\
& ${ }^{12} \mathrm{CO} v(1-0) \mathrm{P} 11$ & $1.34 \times 10^{-13}$ & $4.89 \times 10^{-15}$ \\
& ${ }^{12} \mathrm{CO} v(1-0) \mathrm{P} 12$ & $1.52 \times 10^{-13}$ & $5.89 \times 10^{-15}$ \\
${ }^{12} \mathrm{CO} v(1-0) \mathrm{P} 14$ & $1.50 \times 10^{-13}$ & $6.38 \times 10^{-15}$ \\
\hline
\end{tabular}

Notes. Line equivalent widths are converted to line fluxes using the conversion values listed in Table B.1.
Table B.4. continued.

\begin{tabular}{|c|c|c|c|}
\hline Target & Line id & $\begin{array}{l}\text { Line flux } \\
{\left[\mathrm{erg} \mathrm{cm} \mathrm{cm}^{-2} \mathrm{~s}^{-1} \text { ] }\right.}\end{array}$ & $\begin{array}{l}\text { Error } \\
{\left[\mathrm{erg} \mathrm{cm}^{-2} \mathrm{~s}^{-1}\right]}\end{array}$ \\
\hline & ${ }^{12} \mathrm{CO} v(1-0) \mathrm{P} 30$ & $7.09 \times 10^{-14}$ & $3.06 \times 10^{-15}$ \\
\hline & ${ }^{12} \mathrm{CO} v(1-0) \mathrm{P} 32$ & $5.07 \times 10^{-14}$ & $8.10 \times 10^{-15}$ \\
\hline & ${ }^{12} \mathrm{CO} v(1-0) \mathrm{P} 32$ & $5.07 \times 10^{-14}$ & $7.12 \times 10^{-15}$ \\
\hline & ${ }^{12} \mathrm{CO} v(1-0) \mathrm{P} 36$ & $4.84 \times 10^{-14}$ & $1.32 \times 10^{-14}$ \\
\hline & ${ }^{12} \mathrm{CO} v(1-0) \mathrm{P} 36$ & $3.82 \times 10^{-14}$ & $9.41 \times 10^{-15}$ \\
\hline & ${ }^{12} \mathrm{CO} v(1-0) \mathrm{P} 37$ & $3.83 \times 10^{-14}$ & $5.42 \times 10^{-15}$ \\
\hline & ${ }^{12} \mathrm{CO} v(1-0) \mathrm{P} 05$ & $1.12 \times 10^{-13}$ & $4.25 \times 10^{-15}$ \\
\hline & ${ }^{12} \mathrm{CO} v(1-0) \mathrm{P} 07$ & $1.24 \times 10^{-13}$ & $6.33 \times 10^{-15}$ \\
\hline & ${ }^{12} \mathrm{CO} v(1-0) \mathrm{P} 08$ & $1.34 \times 10^{-13}$ & $5.18 \times 10^{-15}$ \\
\hline & ${ }^{12} \mathrm{CO} v(1-0) \mathrm{P} 08$ & $1.41 \times 10^{-13}$ & $4.85 \times 10^{-15}$ \\
\hline & ${ }^{12} \mathrm{CO} v(1-0) \mathrm{P} 11$ & $1.45 \times 10^{-13}$ & $4.98 \times 10^{-15}$ \\
\hline \multirow[t]{8}{*}{ HD 100546} & ${ }^{13} \mathrm{CO} v(1-0) \mathrm{R} 18$ & $1.07 \times 10^{-14}$ & $1.22 \times 10^{-15}$ \\
\hline & ${ }^{13} \mathrm{CO} v(1-0) \mathrm{R} 15$ & $1.08 \times 10^{-14}$ & $1.92 \times 10^{-15}$ \\
\hline & ${ }^{13} \mathrm{CO} v(1-0) \mathrm{R} 15$ & $1.61 \times 10^{-14}$ & $8.01 \times 10^{-16}$ \\
\hline & ${ }^{13} \mathrm{CO} v(1-0) \mathrm{R} 13$ & $1.58 \times 10^{-14}$ & $7.92 \times 10^{-16}$ \\
\hline & ${ }^{13} \mathrm{CO} v(1-0) \mathrm{R} 12$ & $1.33 \times 10^{-14}$ & $2.35 \times 10^{-15}$ \\
\hline & ${ }^{13} \mathrm{CO} v(1-0) \mathrm{R} 12$ & $1.75 \times 10^{-14}$ & $2.64 \times 10^{-15}$ \\
\hline & ${ }^{13} \mathrm{CO} v(1-0) \mathrm{R} 09$ & $1.58 \times 10^{-14}$ & $2.04 \times 10^{-15}$ \\
\hline & ${ }^{13} \mathrm{CO} v(1-0) \mathrm{P} 23$ & $1.07 \times 10^{-14}$ & $7.33 \times 10^{-16}$ \\
\hline \multirow[t]{19}{*}{ HD 100546} & ${ }^{12} \mathrm{CO} v(2-1) \mathrm{R} 17$ & $3.64 \times 10^{-14}$ & $1.93 \times 10^{-15}$ \\
\hline & ${ }^{12} \mathrm{CO} v(2-1) \mathrm{R} 16$ & $5.13 \times 10^{-14}$ & $4.46 \times 10^{-15}$ \\
\hline & ${ }^{12} \mathrm{CO} v(2-1) \mathrm{R} 14$ & $3.97 \times 10^{-14}$ & $2.39 \times 10^{-15}$ \\
\hline & ${ }^{12} \mathrm{CO} v(2-1) \mathrm{R} 14$ & $4.35 \times 10^{-14}$ & $3.75 \times 10^{-15}$ \\
\hline & ${ }^{12} \mathrm{CO} v(2-1) \mathrm{R} 12$ & $5.42 \times 10^{-14}$ & $7.79 \times 10^{-15}$ \\
\hline & ${ }^{12} \mathrm{CO} v(2-1) \mathrm{R} 12$ & $6.18 \times 10^{-14}$ & $2.64 \times 10^{-15}$ \\
\hline & ${ }^{12} \mathrm{CO} v(2-1) \mathrm{R} 11$ & $5.47 \times 10^{-14}$ & $2.20 \times 10^{-15}$ \\
\hline & ${ }^{12} \mathrm{CO} v(2-1) \mathrm{R} 10$ & $5.90 \times 10^{-14}$ & $2.35 \times 10^{-15}$ \\
\hline & ${ }^{12} \mathrm{CO} v(2-1) \mathrm{R} 10$ & $6.03 \times 10^{-14}$ & $2.30 \times 10^{-15}$ \\
\hline & ${ }^{12} \mathrm{CO} v(2-1) \mathrm{R} 09$ & $5.14 \times 10^{-14}$ & $2.75 \times 10^{-15}$ \\
\hline & ${ }^{12} \mathrm{CO} v(2-1) \mathrm{R} 06$ & $5.61 \times 10^{-14}$ & $2.63 \times 10^{-15}$ \\
\hline & ${ }^{12} \mathrm{CO} v(2-1) \mathrm{R} 06$ & $5.56 \times 10^{-14}$ & $2.36 \times 10^{-15}$ \\
\hline & ${ }^{12} \mathrm{CO} v(2-1) \mathrm{R} 05$ & $4.62 \times 10^{-14}$ & $2.09 \times 10^{-15}$ \\
\hline & ${ }^{12} \mathrm{CO} v(2-1) \mathrm{R} 04$ & $3.45 \times 10^{-14}$ & $2.02 \times 10^{-15}$ \\
\hline & ${ }^{12} \mathrm{CO} v(2-1) \mathrm{P} 25$ & $3.54 \times 10^{-14}$ & $5.15 \times 10^{-15}$ \\
\hline & ${ }^{12} \mathrm{CO} v(2-1) \mathrm{P} 25$ & $3.65 \times 10^{-14}$ & $2.46 \times 10^{-15}$ \\
\hline & ${ }^{12} \mathrm{CO} v(2-1) \mathrm{P} 26$ & $3.64 \times 10^{-14}$ & $3.46 \times 10^{-15}$ \\
\hline & ${ }^{12} \mathrm{CO} v(2-1) \mathrm{P} 28$ & $2.66 \times 10^{-14}$ & $2.36 \times 10^{-15}$ \\
\hline & ${ }^{12} \mathrm{CO} v(2-1) \mathrm{P} 28$ & $3.06 \times 10^{-14}$ & $3.99 \times 10^{-15}$ \\
\hline \multirow{15}{*}{ HD 100546} & ${ }^{12} \mathrm{CO} v(3-2) \mathrm{R} 25$ & $2.11 \times 10^{-14}$ & $1.63 \times 10^{-15}$ \\
\hline & ${ }^{12} \mathrm{CO} v(3-2) \mathrm{R} 23$ & $2.21 \times 10^{-14}$ & $1.11 \times 10^{-15}$ \\
\hline & ${ }^{12} \mathrm{CO} v(3-2) \mathrm{R} 23$ & $2.03 \times 10^{-14}$ & $1.03 \times 10^{-15}$ \\
\hline & ${ }^{12} \mathrm{CO} v(3-2) \mathrm{R} 20$ & $2.12 \times 10^{-14}$ & $1.19 \times 10^{-15}$ \\
\hline & ${ }^{12} \mathrm{CO} v(3-2) \mathrm{R} 18$ & $2.64 \times 10^{-14}$ & $1.56 \times 10^{-15}$ \\
\hline & ${ }^{12} \mathrm{CO} v(3-2) \mathrm{R} 17$ & $3.31 \times 10^{-14}$ & $1.90 \times 10^{-15}$ \\
\hline & ${ }^{12} \mathrm{CO} v(3-2) \mathrm{R} 14$ & $3.70 \times 10^{-14}$ & $1.50 \times 10^{-15}$ \\
\hline & ${ }^{12} \mathrm{CO} v(3-2) \mathrm{R} 14$ & $3.90 \times 10^{-14}$ & $1.44 \times 10^{-15}$ \\
\hline & ${ }^{12} \mathrm{CO} v(3-2) \mathrm{R} 11$ & $3.93 \times 10^{-14}$ & $1.59 \times 10^{-15}$ \\
\hline & ${ }^{12} \mathrm{CO} v(3-2) \mathrm{R} 08$ & $3.86 \times 10^{-14}$ & $2.96 \times 10^{-15}$ \\
\hline & ${ }^{12} \mathrm{CO} v(3-2) \mathrm{R} 08$ & $3.49 \times 10^{-14}$ & $2.09 \times 10^{-15}$ \\
\hline & ${ }^{12} \mathrm{CO} v(3-2) \mathrm{R} 05$ & $3.19 \times 10^{-14}$ & $3.00 \times 10^{-15}$ \\
\hline & ${ }^{12} \mathrm{CO} v(3-2) \mathrm{R} 01$ & $2.08 \times 10^{-14}$ & $4.52 \times 10^{-15}$ \\
\hline & ${ }^{12} \mathrm{CO} v(3-2) \mathrm{P} 21$ & $2.07 \times 10^{-14}$ & $1.70 \times 10^{-15}$ \\
\hline & ${ }^{12} \mathrm{CO} v(3-2) \mathrm{P} 21$ & $2.19 \times 10^{-14}$ & $3.91 \times 10^{-15}$ \\
\hline \multirow[t]{8}{*}{ HD 100546} & ${ }^{12} \mathrm{CO} v(4-3) \mathrm{R} 32$ & $1.20 \times 10^{-14}$ & $1.77 \times 10^{-15}$ \\
\hline & ${ }^{12} \mathrm{CO} v(4-3) \mathrm{R} 27$ & $1.84 \times 10^{-14}$ & $1.62 \times 10^{-15}$ \\
\hline & ${ }^{12} \mathrm{CO} v(4-3) \mathrm{R} 27$ & $1.48 \times 10^{-14}$ & $5.04 \times 10^{-15}$ \\
\hline & ${ }^{12} \mathrm{CO} v(4-3) \mathrm{R} 23$ & $1.82 \times 10^{-14}$ & $2.14 \times 10^{-15}$ \\
\hline & ${ }^{12} \mathrm{CO} v(4-3) \mathrm{R} 23$ & $2.28 \times 10^{-14}$ & $1.86 \times 10^{-15}$ \\
\hline & ${ }^{12} \mathrm{CO} v(4-3) \mathrm{R} 19$ & $2.38 \times 10^{-14}$ & $3.20 \times 10^{-15}$ \\
\hline & ${ }^{12} \mathrm{CO} v(4-3) \mathrm{R} 19$ & $2.21 \times 10^{-14}$ & $2.19 \times 10^{-15}$ \\
\hline & ${ }^{12} \mathrm{CO} v(4-3) \mathrm{R} 16$ & $2.45 \times 10^{-14}$ & $3.45 \times 10^{-15}$ \\
\hline
\end{tabular}


Table B.4. continued.

\begin{tabular}{|c|c|c|c|}
\hline Target & Line id & $\begin{array}{l}\text { Line flux } \\
{\left[\mathrm{erg} \mathrm{cm}^{-2} \mathrm{~s}^{-1} \text { ] }\right.}\end{array}$ & $\begin{array}{l}\text { Error } \\
{\left[\mathrm{erg} \mathrm{cm}^{-2} \mathrm{~s}^{-1}\right]}\end{array}$ \\
\hline & ${ }^{12} \mathrm{CO} v(4-3) \mathrm{R} 15$ & $2.44 \times 10^{-14}$ & $3.77 \times 10^{-15}$ \\
\hline & ${ }^{12} \mathrm{CO} v(4-3) \mathrm{R} 13$ & $2.65 \times 10^{-14}$ & $2.47 \times 10^{-15}$ \\
\hline & ${ }^{12} \mathrm{CO} v(4-3) \mathrm{R} 12$ & $3.10 \times 10^{-14}$ & $3.53 \times 10^{-15}$ \\
\hline & ${ }^{12} \mathrm{CO} v(4-3) \mathrm{R} 11$ & $2.99 \times 10^{-14}$ & $3.57 \times 10^{-15}$ \\
\hline & ${ }^{12} \mathrm{CO} v(4-3) \mathrm{R} 10$ & $2.79 \times 10^{-14}$ & $8.46 \times 10^{-15}$ \\
\hline & ${ }^{12} \mathrm{CO} v(4-3) \mathrm{R} 08$ & $2.64 \times 10^{-14}$ & $2.71 \times 10^{-15}$ \\
\hline & ${ }^{12} \mathrm{CO} v(4-3) \mathrm{P} 17$ & $2.41 \times 10^{-14}$ & $3.41 \times 10^{-15}$ \\
\hline & ${ }^{12} \mathrm{CO} v(4-3) \mathrm{P} 17$ & $2.31 \times 10^{-14}$ & $2.53 \times 10^{-15}$ \\
\hline \multirow{11}{*}{ HD 100546} & ${ }^{12} \mathrm{CO} v(5-4) \mathrm{R} 39$ & $1.00 \times 10^{-14}$ & $4.68 \times 10^{-15}$ \\
\hline & ${ }^{12} \mathrm{CO} v(5-4) \mathrm{R} 35$ & $9.75 \times 10^{-15}$ & $3.92 \times 10^{-15}$ \\
\hline & ${ }^{12} \mathrm{CO} v(5-4) \mathrm{R} 34$ & $6.59 \times 10^{-15}$ & $3.78 \times 10^{-15}$ \\
\hline & ${ }^{12} \mathrm{CO} v(5-4) \mathrm{R} 33$ & $8.96 \times 10^{-15}$ & $4.76 \times 10^{-15}$ \\
\hline & ${ }^{12} \mathrm{CO} v(5-4) \mathrm{R} 33$ & $6.06 \times 10^{-15}$ & $3.23 \times 10^{-15}$ \\
\hline & ${ }^{12} \mathrm{CO} v(5-4) \mathrm{R} 27$ & $1.69 \times 10^{-14}$ & $7.18 \times 10^{-15}$ \\
\hline & ${ }^{12} \mathrm{CO} v(5-4) \mathrm{P} 06$ & $2.58 \times 10^{-14}$ & $7.45 \times 10^{-15}$ \\
\hline & ${ }^{12} \mathrm{CO} v(5-4) \mathrm{P} 07$ & $3.15 \times 10^{-14}$ & $5.50 \times 10^{-15}$ \\
\hline & ${ }^{12} \mathrm{CO} v(5-4) \mathrm{P} 07$ & $1.94 \times 10^{-14}$ & $1.39 \times 10^{-15}$ \\
\hline & ${ }^{12} \mathrm{CO} v(5-4) \mathrm{P} 09$ & $1.34 \times 10^{-14}$ & $1.50 \times 10^{-15}$ \\
\hline & ${ }^{12} \mathrm{CO} v(5-4) \mathrm{P} 09$ & $2.07 \times 10^{-14}$ & $1.36 \times 10^{-15}$ \\
\hline \multirow[t]{19}{*}{ HD 97048} & ${ }^{12} \mathrm{CO} v(1-0) \mathrm{R} 08$ & $1.06 \times 10^{-13}$ & $1.42 \times 10^{-14}$ \\
\hline & ${ }^{12} \mathrm{CO} v(1-0) \mathrm{R} 07$ & $9.97 \times 10^{-14}$ & $8.45 \times 10^{-15}$ \\
\hline & ${ }^{12} \mathrm{CO} v(1-0) \mathrm{R} 07$ & $9.53 \times 10^{-14}$ & $7.90 \times 10^{-15}$ \\
\hline & ${ }^{12} \mathrm{CO} v(1-0) \mathrm{R} 06$ & $1.15 \times 10^{-13}$ & $9.49 \times 10^{-15}$ \\
\hline & ${ }^{12} \mathrm{CO} v(1-0) \mathrm{R} 06$ & $1.05 \times 10^{-13}$ & $8.57 \times 10^{-15}$ \\
\hline & ${ }^{12} \mathrm{CO} v(1-0) \mathrm{P} 05$ & $1.21 \times 10^{-13}$ & $4.99 \times 10^{-15}$ \\
\hline & ${ }^{12} \mathrm{CO} v(1-0) \mathrm{P} 05$ & $1.31 \times 10^{-13}$ & $1.84 \times 10^{-14}$ \\
\hline & ${ }^{12} \mathrm{CO} v(1-0) \mathrm{P} 07$ & $1.18 \times 10^{-13}$ & $9.91 \times 10^{-15}$ \\
\hline & ${ }^{12} \mathrm{CO} v(1-0) \mathrm{P} 07$ & $1.01 \times 10^{-13}$ & $8.47 \times 10^{-15}$ \\
\hline & ${ }^{12} \mathrm{CO} v(1-0) \mathrm{P} 08$ & $1.23 \times 10^{-13}$ & $1.01 \times 10^{-14}$ \\
\hline & ${ }^{12} \mathrm{CO} v(1-0) \mathrm{P} 08$ & $1.22 \times 10^{-13}$ & $1.02 \times 10^{-14}$ \\
\hline & ${ }^{12} \mathrm{CO} v(1-0) \mathrm{P} 09$ & $1.07 \times 10^{-13}$ & $8.99 \times 10^{-15}$ \\
\hline & ${ }^{12} \mathrm{CO} v(1-0) \mathrm{P} 11$ & $1.35 \times 10^{-13}$ & $1.13 \times 10^{-14}$ \\
\hline & ${ }^{12} \mathrm{CO} v(1-0) \mathrm{P} 11$ & $1.11 \times 10^{-13}$ & $9.33 \times 10^{-15}$ \\
\hline & ${ }^{12} \mathrm{CO} v(1-0) \mathrm{P} 12$ & $1.40 \times 10^{-13}$ & $7.85 \times 10^{-15}$ \\
\hline & ${ }^{12} \mathrm{CO} v(1-0) \mathrm{P} 14$ & $1.21 \times 10^{-13}$ & $2.87 \times 10^{-14}$ \\
\hline & ${ }^{12} \mathrm{CO} v(1-0) \mathrm{P} 30$ & $3.45 \times 10^{-14}$ & $9.24 \times 10^{-15}$ \\
\hline & ${ }^{12} \mathrm{CO} v(1-0) \mathrm{P} 32$ & $2.60 \times 10^{-14}$ & $6.68 \times 10^{-15}$ \\
\hline & ${ }^{12} \mathrm{CO} v(1-0) \mathrm{P} 36$ & $1.34 \times 10^{-14}$ & $7.66 \times 10^{-15}$ \\
\hline \multirow[t]{9}{*}{ HD 97048} & ${ }^{13} \mathrm{CO} v(1-0) \mathrm{R} 15$ & $2.02 \times 10^{-14}$ & $2.07 \times 10^{-15}$ \\
\hline & ${ }^{13} \mathrm{CO} v(1-0) \mathrm{R} 13$ & $1.91 \times 10^{-14}$ & $1.52 \times 10^{-15}$ \\
\hline & ${ }^{13} \mathrm{CO} v(1-0) \mathrm{R} 12$ & $2.04 \times 10^{-14}$ & $2.74 \times 10^{-15}$ \\
\hline & ${ }^{13} \mathrm{CO} v(1-0) \mathrm{R} 12$ & $1.71 \times 10^{-14}$ & $1.68 \times 10^{-15}$ \\
\hline & ${ }^{13} \mathrm{CO} v(1-0) \mathrm{R} 09$ & $2.35 \times 10^{-14}$ & $3.77 \times 10^{-15}$ \\
\hline & ${ }^{13} \mathrm{CO} v(1-0) \mathrm{R} 09$ & $1.46 \times 10^{-14}$ & $1.98 \times 10^{-15}$ \\
\hline & ${ }^{13} \mathrm{CO} v(1-0) \mathrm{R} 06$ & $3.06 \times 10^{-14}$ & $3.51 \times 10^{-15}$ \\
\hline & ${ }^{13} \mathrm{CO} v(1-0) \mathrm{R} 05$ & $2.93 \times 10^{-14}$ & $2.31 \times 10^{-15}$ \\
\hline & ${ }^{13} \mathrm{CO} v(1-0) \mathrm{R} 04$ & $1.57 \times 10^{-14}$ & $1.75 \times 10^{-15}$ \\
\hline \multirow{13}{*}{ HD 97048} & ${ }^{12} \mathrm{CO} v(2-1) \mathrm{R} 17$ & $4.12 \times 10^{-14}$ & $3.39 \times 10^{-15}$ \\
\hline & ${ }^{12} \mathrm{CO} v(2-1) \mathrm{R} 14$ & $3.97 \times 10^{-14}$ & $3.05 \times 10^{-15}$ \\
\hline & ${ }^{12} \mathrm{CO} v(2-1) \mathrm{R} 13$ & $4.31 \times 10^{-14}$ & $2.50 \times 10^{-15}$ \\
\hline & ${ }^{12} \mathrm{CO} v(2-1) \mathrm{R} 12$ & $4.36 \times 10^{-14}$ & $2.27 \times 10^{-15}$ \\
\hline & ${ }^{12} \mathrm{CO} v(2-1) \mathrm{R} 11$ & $4.05 \times 10^{-14}$ & $2.19 \times 10^{-15}$ \\
\hline & ${ }^{12} \mathrm{CO} v(2-1) \mathrm{R} 10$ & $3.83 \times 10^{-14}$ & $1.78 \times 10^{-15}$ \\
\hline & ${ }^{12} \mathrm{CO} v(2-1) \mathrm{R} 09$ & $4.05 \times 10^{-14}$ & $1.62 \times 10^{-15}$ \\
\hline & ${ }^{12} \mathrm{CO} v(2-1) \mathrm{R} 06$ & $3.85 \times 10^{-14}$ & $1.58 \times 10^{-15}$ \\
\hline & ${ }^{12} \mathrm{CO} v(2-1) \mathrm{R} 06$ & $3.89 \times 10^{-14}$ & $1.37 \times 10^{-15}$ \\
\hline & ${ }^{12} \mathrm{CO} v(2-1) \mathrm{R} 05$ & $4.98 \times 10^{-14}$ & $2.39 \times 10^{-15}$ \\
\hline & ${ }^{12} \mathrm{CO} v(2-1) \mathrm{R} 04$ & $3.91 \times 10^{-14}$ & $2.24 \times 10^{-15}$ \\
\hline & ${ }^{12} \mathrm{CO} v(2-1) \mathrm{R} 04$ & $4.03 \times 10^{-14}$ & $1.92 \times 10^{-15}$ \\
\hline & ${ }^{12} \mathrm{CO} v(2-1) \mathrm{P} 02$ & $3.20 \times 10^{-14}$ & $3.59 \times 10^{-15}$ \\
\hline
\end{tabular}

Table B.4. continued.

\begin{tabular}{|c|c|c|c|}
\hline Target & Line id & $\begin{array}{l}\text { Line flux } \\
{\left[\mathrm{erg} \mathrm{cm}^{-2} \mathrm{~s}^{-1}\right]}\end{array}$ & $\begin{array}{l}\text { Error } \\
{\left[\mathrm{erg} \mathrm{cm}^{-2} \mathrm{~s}^{-1}\right]}\end{array}$ \\
\hline & ${ }^{12} \mathrm{CO} v(2-1) \mathrm{P} 02$ & $3.03 \times 10^{-14}$ & $4.25 \times 10^{-15}$ \\
\hline & ${ }^{12} \mathrm{CO} v(2-1) \mathrm{P} 07$ & $4.57 \times 10^{-14}$ & $1.39 \times 10^{-14}$ \\
\hline \multirow[t]{12}{*}{ HD 97048} & ${ }^{12} \mathrm{CO} v(3-2) \mathrm{R} 26$ & $2.65 \times 10^{-14}$ & $2.12 \times 10^{-15}$ \\
\hline & ${ }^{12} \mathrm{CO} v(3-2) \mathrm{R} 22$ & $3.06 \times 10^{-14}$ & $2.99 \times 10^{-15}$ \\
\hline & ${ }^{12} \mathrm{CO} v(3-2) \mathrm{R} 20$ & $2.20 \times 10^{-14}$ & $1.68 \times 10^{-15}$ \\
\hline & ${ }^{12} \mathrm{CO} v(3-2) \mathrm{R} 18$ & $3.15 \times 10^{-14}$ & $2.81 \times 10^{-15}$ \\
\hline & ${ }^{12} \mathrm{CO} v(3-2) \mathrm{R} 18$ & $2.77 \times 10^{-14}$ & $2.08 \times 10^{-15}$ \\
\hline & ${ }^{12} \mathrm{CO} v(3-2) \mathrm{R} 17$ & $2.25 \times 10^{-14}$ & $1.64 \times 10^{-15}$ \\
\hline & ${ }^{12} \mathrm{CO} v(3-2) \mathrm{R} 14$ & $3.23 \times 10^{-14}$ & $2.46 \times 10^{-15}$ \\
\hline & ${ }^{12} \mathrm{CO} v(3-2) \mathrm{R} 14$ & $2.25 \times 10^{-14}$ & $5.89 \times 10^{-15}$ \\
\hline & ${ }^{12} \mathrm{CO} v(3-2) \mathrm{R} 11$ & $3.16 \times 10^{-14}$ & $2.89 \times 10^{-15}$ \\
\hline & ${ }^{12} \mathrm{CO} v(3-2) \mathrm{R} 08$ & $3.99 \times 10^{-14}$ & $4.93 \times 10^{-15}$ \\
\hline & ${ }^{12} \mathrm{CO} v(3-2) \mathrm{R} 08$ & $4.68 \times 10^{-14}$ & $3.79 \times 10^{-15}$ \\
\hline & ${ }^{12} \mathrm{CO} v(3-2) \mathrm{R} 05$ & $1.90 \times 10^{-14}$ & $2.30 \times 10^{-15}$ \\
\hline \multirow[t]{10}{*}{ HD 97048} & ${ }^{12} \mathrm{CO} v(4-3) \mathrm{R} 27$ & $1.11 \times 10^{-14}$ & $5.01 \times 10^{-15}$ \\
\hline & ${ }^{12} \mathrm{CO} v(4-3) \mathrm{R} 23$ & $2.04 \times 10^{-14}$ & $10.00 \times 10^{-15}$ \\
\hline & ${ }^{12} \mathrm{CO} v(4-3) \mathrm{R} 16$ & $3.39 \times 10^{-14}$ & $3.55 \times 10^{-15}$ \\
\hline & ${ }^{12} \mathrm{CO} v(4-3) \mathrm{R} 15$ & $4.14 \times 10^{-14}$ & $7.01 \times 10^{-15}$ \\
\hline & ${ }^{12} \mathrm{CO} v(4-3) \mathrm{R} 13$ & $3.16 \times 10^{-14}$ & $3.64 \times 10^{-15}$ \\
\hline & ${ }^{12} \mathrm{CO} v(4-3) \mathrm{R} 11$ & $2.13 \times 10^{-14}$ & $1.08 \times 10^{-14}$ \\
\hline & ${ }^{12} \mathrm{CO} v(4-3) \mathrm{R} 09$ & $1.81 \times 10^{-14}$ & $2.56 \times 10^{-15}$ \\
\hline & ${ }^{12} \mathrm{CO} v(4-3) \mathrm{R} 08$ & $3.70 \times 10^{-14}$ & $3.10 \times 10^{-15}$ \\
\hline & ${ }^{12} \mathrm{CO} v(4-3) \mathrm{P} 15$ & $1.99 \times 10^{-14}$ & $6.46 \times 10^{-15}$ \\
\hline & ${ }^{12} \mathrm{CO} v(4-3) \mathrm{P} 17$ & $2.53 \times 10^{-14}$ & $8.85 \times 10^{-15}$ \\
\hline \multirow[t]{4}{*}{ HD 97048} & ${ }^{12} \mathrm{CO} v(5-4) \mathrm{R} 33$ & $1.16 \times 10^{-14}$ & $4.73 \times 10^{-15}$ \\
\hline & ${ }^{12} \mathrm{CO} v(5-4) \mathrm{R} 15$ & $2.13 \times 10^{-14}$ & $1.11 \times 10^{-14}$ \\
\hline & ${ }^{12} \mathrm{CO} v(5-4) \mathrm{P} 06$ & $3.15 \times 10^{-14}$ & $1.63 \times 10^{-14}$ \\
\hline & ${ }^{12} \mathrm{CO} v(5-4) \mathrm{P} 09$ & $2.60 \times 10^{-14}$ & $1.58 \times 10^{-14}$ \\
\hline \multirow[t]{7}{*}{ HD 179218} & ${ }^{12} \mathrm{CO} v(1-0) \mathrm{R} 06$ & $2.96 \times 10^{-14}$ & $4.07 \times 10^{-15}$ \\
\hline & ${ }^{12} \mathrm{CO} v(1-0) \mathrm{R} 03$ & $2.06 \times 10^{-14}$ & $2.91 \times 10^{-15}$ \\
\hline & ${ }^{12} \mathrm{CO} v(1-0) \mathrm{P} 10$ & $2.45 \times 10^{-14}$ & $3.39 \times 10^{-15}$ \\
\hline & ${ }^{12} \mathrm{CO} v(1-0) \mathrm{P} 12$ & $3.31 \times 10^{-14}$ & $1.44 \times 10^{-14}$ \\
\hline & ${ }^{12} \mathrm{CO} v(1-0) \mathrm{P} 30$ & $1.48 \times 10^{-14}$ & $1.13 \times 10^{-15}$ \\
\hline & ${ }^{12} \mathrm{CO} v(1-0) \mathrm{P} 32$ & $1.17 \times 10^{-14}$ & $9.78 \times 10^{-16}$ \\
\hline & ${ }^{12} \mathrm{CO} v(1-0) \mathrm{P} 36$ & $8.56 \times 10^{-15}$ & $1.17 \times 10^{-15}$ \\
\hline \multirow[t]{7}{*}{ HD 179218} & ${ }^{13} \mathrm{CO} v(1-0) \mathrm{R} 21$ & $5.53 \times 10^{-15}$ & $7.27 \times 10^{-16}$ \\
\hline & ${ }^{13} \mathrm{CO} v(1-0) \mathrm{R} 15$ & $8.43 \times 10^{-15}$ & $1.01 \times 10^{-15}$ \\
\hline & ${ }^{13} \mathrm{CO} v(1-0) \mathrm{R} 12$ & $1.07 \times 10^{-14}$ & $9.01 \times 10^{-16}$ \\
\hline & ${ }^{13} \mathrm{CO} v(1-0) \mathrm{R} 12$ & $9.88 \times 10^{-15}$ & $9.16 \times 10^{-16}$ \\
\hline & ${ }^{13} \mathrm{CO} v(1-0) \mathrm{R} 03$ & $8.04 \times 10^{-15}$ & $2.40 \times 10^{-15}$ \\
\hline & ${ }^{13} \mathrm{CO} v(1-0) \mathrm{P} 21$ & $6.19 \times 10^{-15}$ & $5.37 \times 10^{-16}$ \\
\hline & ${ }^{13} \mathrm{CO} v(1-0) \mathrm{P} 21$ & $8.17 \times 10^{-15}$ & $9.10 \times 10^{-16}$ \\
\hline \multirow[t]{10}{*}{ HD 179218} & ${ }^{12} \mathrm{CO} v(2-1) \mathrm{R} 14$ & $1.16 \times 10^{-14}$ & $7.95 \times 10^{-16}$ \\
\hline & ${ }^{12} \mathrm{CO} v(2-1) \mathrm{R} 13$ & $1.09 \times 10^{-14}$ & $6.59 \times 10^{-16}$ \\
\hline & ${ }^{12} \mathrm{CO} v(2-1) \mathrm{R} 09$ & $1.17 \times 10^{-14}$ & $9.08 \times 10^{-16}$ \\
\hline & ${ }^{12} \mathrm{CO} v(2-1) \mathrm{R} 08$ & $1.08 \times 10^{-14}$ & $8.24 \times 10^{-16}$ \\
\hline & ${ }^{12} \mathrm{CO} v(2-1) \mathrm{R} 06$ & $1.08 \times 10^{-14}$ & $7.12 \times 10^{-16}$ \\
\hline & ${ }^{12} \mathrm{CO} v(2-1) \mathrm{R} 04$ & $5.93 \times 10^{-15}$ & $7.27 \times 10^{-16}$ \\
\hline & ${ }^{12} \mathrm{CO} v(2-1) \mathrm{P} 25$ & $8.30 \times 10^{-15}$ & $1.44 \times 10^{-15}$ \\
\hline & ${ }^{12} \mathrm{CO} v(2-1) \mathrm{P} 26$ & $1.08 \times 10^{-14}$ & $2.54 \times 10^{-15}$ \\
\hline & ${ }^{12} \mathrm{CO} v(2-1) \mathrm{P} 28$ & $8.30 \times 10^{-15}$ & $1.01 \times 10^{-15}$ \\
\hline & ${ }^{12} \mathrm{CO} v(2-1) \mathrm{P} 28$ & $5.80 \times 10^{-15}$ & $6.15 \times 10^{-16}$ \\
\hline \multirow[t]{7}{*}{ HD 179218} & ${ }^{12} \mathrm{CO} v(3-2) \mathrm{R} 23$ & $9.22 \times 10^{-15}$ & $7.57 \times 10^{-16}$ \\
\hline & ${ }^{12} \mathrm{CO} v(3-2) \mathrm{R} 20$ & $1.11 \times 10^{-14}$ & $1.90 \times 10^{-15}$ \\
\hline & ${ }^{12} \mathrm{CO} v(3-2) \mathrm{R} 20$ & $1.03 \times 10^{-14}$ & $8.03 \times 10^{-16}$ \\
\hline & ${ }^{12} \mathrm{CO} v(3-2) \mathrm{R} 18$ & $8.96 \times 10^{-15}$ & $7.59 \times 10^{-16}$ \\
\hline & ${ }^{12} \mathrm{CO} v(3-2) \mathrm{R} 14$ & $1.24 \times 10^{-14}$ & $3.19 \times 10^{-15}$ \\
\hline & ${ }^{12} \mathrm{CO} v(3-2) \mathrm{P} 21$ & $9.09 \times 10^{-15}$ & $8.27 \times 10^{-16}$ \\
\hline & ${ }^{12} \mathrm{CO} v(3-2) \mathrm{P} 21$ & $9.88 \times 10^{-15}$ & $5.48 \times 10^{-16}$ \\
\hline
\end{tabular}


G. van der Plas et al.: CO ro-vibrational line survey of HAeBe disks

Table B.4. continued.

\begin{tabular}{|c|c|c|c|}
\hline Target & Line id & $\begin{array}{l}\text { Line flux } \\
{\left[\mathrm{erg} \mathrm{cm}^{-2} \mathrm{~s}^{-1}\right]}\end{array}$ & $\begin{array}{l}\text { Error } \\
{\left[\mathrm{erg} \mathrm{cm}^{-2} \mathrm{~s}^{-1}\right]}\end{array}$ \\
\hline \multirow[t]{6}{*}{ HD 179218} & ${ }^{12} \mathrm{CO} v(4-3) \mathrm{R} 27$ & $6.32 \times 10^{-15}$ & $2.80 \times 10^{-15}$ \\
\hline & ${ }^{12} \mathrm{CO} v(4-3) \mathrm{R} 26$ & $8.04 \times 10^{-15}$ & $3.29 \times 10^{-15}$ \\
\hline & ${ }^{12} \mathrm{CO} v(4-3) \mathrm{R} 23$ & $8.56 \times 10^{-15}$ & $4.85 \times 10^{-15}$ \\
\hline & ${ }^{12} \mathrm{CO} v(4-3) \mathrm{R} 23$ & $1.00 \times 10^{-14}$ & $3.72 \times 10^{-15}$ \\
\hline & ${ }^{12} \mathrm{CO} v(4-3) \mathrm{P} 17$ & $9.62 \times 10^{-15}$ & $1.57 \times 10^{-15}$ \\
\hline & ${ }^{12} \mathrm{CO} v(4-3) \mathrm{P} 20$ & $7.64 \times 10^{-15}$ & $1.26 \times 10^{-15}$ \\
\hline \multirow[t]{3}{*}{ HD 141569} & ${ }^{13} \mathrm{CO} v(1-0) \mathrm{R} 09$ & $2.12 \times 10^{-14}$ & $8.18 \times 10^{-15}$ \\
\hline & ${ }^{13} \mathrm{CO} v(1-0) \mathrm{R} 04$ & $1.91 \times 10^{-14}$ & $7.44 \times 10^{-15}$ \\
\hline & ${ }^{13} \mathrm{CO} v(1-0) \mathrm{R} 00$ & $2.42 \times 10^{-14}$ & $1.06 \times 10^{-14}$ \\
\hline \multirow[t]{8}{*}{ HD 141569} & ${ }^{12} \mathrm{CO} v(2-1) \mathrm{R} 14$ & $2.42 \times 10^{-14}$ & $8.32 \times 10^{-15}$ \\
\hline & ${ }^{12} \mathrm{CO} v(2-1) \mathrm{R} 11$ & $5.85 \times 10^{-14}$ & $1.41 \times 10^{-14}$ \\
\hline & ${ }^{12} \mathrm{CO} v(2-1) \mathrm{R} 10$ & $5.30 \times 10^{-14}$ & $8.53 \times 10^{-15}$ \\
\hline & ${ }^{12} \mathrm{CO} v(2-1) \mathrm{R} 07$ & $7.13 \times 10^{-14}$ & $1.01 \times 10^{-14}$ \\
\hline & ${ }^{12} \mathrm{CO} v(2-1) \mathrm{R} 06$ & $6.46 \times 10^{-14}$ & $9.17 \times 10^{-15}$ \\
\hline & ${ }^{12} \mathrm{CO} v(2-1) \mathrm{R} 04$ & $8.34 \times 10^{-14}$ & $1.19 \times 10^{-14}$ \\
\hline & ${ }^{12} \mathrm{CO} v(2-1) \mathrm{R} 02$ & $6.73 \times 10^{-14}$ & $9.88 \times 10^{-15}$ \\
\hline & ${ }^{12} \mathrm{CO} v(2-1) \mathrm{P} 01$ & $3.52 \times 10^{-14}$ & $8.77 \times 10^{-15}$ \\
\hline \multirow[t]{28}{*}{ HD 190073} & ${ }^{13} \mathrm{CO} v(1-0) \mathrm{R} 23$ & $1.03 \times 10^{-14}$ & $9.97 \times 10^{-16}$ \\
\hline & ${ }^{13} \mathrm{CO} v(1-0) \mathrm{R} 22$ & $1.19 \times 10^{-14}$ & $6.65 \times 10^{-16}$ \\
\hline & ${ }^{13} \mathrm{CO} v(1-0) \mathrm{R} 21$ & $1.40 \times 10^{-14}$ & $9.13 \times 10^{-16}$ \\
\hline & ${ }^{13} \mathrm{CO} v(1-0) \mathrm{R} 19$ & $1.59 \times 10^{-14}$ & $7.05 \times 10^{-16}$ \\
\hline & ${ }^{13} \mathrm{CO} v(1-0) \mathrm{R} 19$ & $1.49 \times 10^{-14}$ & $6.02 \times 10^{-16}$ \\
\hline & ${ }^{13} \mathrm{CO} v(1-0) \mathrm{R} 18$ & $1.67 \times 10^{-14}$ & $8.95 \times 10^{-16}$ \\
\hline & ${ }^{13} \mathrm{CO} v(1-0) \mathrm{R} 16$ & $1.45 \times 10^{-14}$ & $6.10 \times 10^{-16}$ \\
\hline & ${ }^{13} \mathrm{CO} v(1-0) \mathrm{R} 16$ & $1.07 \times 10^{-14}$ & $1.72 \times 10^{-15}$ \\
\hline & ${ }^{13} \mathrm{CO} v(1-0) \mathrm{R} 15$ & $1.63 \times 10^{-14}$ & $7.12 \times 10^{-16}$ \\
\hline & ${ }^{13} \mathrm{CO} v(1-0) \mathrm{R} 13$ & $2.08 \times 10^{-14}$ & $8.07 \times 10^{-16}$ \\
\hline & ${ }^{13} \mathrm{CO} v(1-0) \mathrm{R} 12$ & $1.74 \times 10^{-14}$ & $7.12 \times 10^{-16}$ \\
\hline & ${ }^{13} \mathrm{CO} v(1-0) \mathrm{R} 12$ & $1.99 \times 10^{-14}$ & $8.52 \times 10^{-16}$ \\
\hline & ${ }^{13} \mathrm{CO} v(1-0) \mathrm{R} 11$ & $1.34 \times 10^{-14}$ & $7.13 \times 10^{-16}$ \\
\hline & ${ }^{13} \mathrm{CO} v(1-0) \mathrm{R} 10$ & $1.77 \times 10^{-14}$ & $6.58 \times 10^{-16}$ \\
\hline & ${ }^{13} \mathrm{CO} v(1-0) \mathrm{R} 10$ & $1.58 \times 10^{-14}$ & $5.98 \times 10^{-16}$ \\
\hline & ${ }^{13} \mathrm{CO} v(1-0) \mathrm{R} 09$ & $1.40 \times 10^{-14}$ & $2.76 \times 10^{-15}$ \\
\hline & ${ }^{13} \mathrm{CO} v(1-0) \mathrm{R} 09$ & $1.62 \times 10^{-14}$ & $4.26 \times 10^{-15}$ \\
\hline & ${ }^{13} \mathrm{CO} v(1-0) \mathrm{R} 06$ & $1.11 \times 10^{-14}$ & $1.21 \times 10^{-15}$ \\
\hline & ${ }^{13} \mathrm{CO} v(1-0) \mathrm{R} 05$ & $1.21 \times 10^{-14}$ & $8.57 \times 10^{-16}$ \\
\hline & ${ }^{13} \mathrm{CO} v(1-0) \mathrm{R} 05$ & $2.36 \times 10^{-14}$ & $8.52 \times 10^{-15}$ \\
\hline & ${ }^{13} \mathrm{CO} v(1-0) \mathrm{R} 03$ & $2.36 \times 10^{-14}$ & $1.22 \times 10^{-15}$ \\
\hline & ${ }^{13} \mathrm{CO} v(1-0) \mathrm{R} 03$ & $1.49 \times 10^{-14}$ & $9.33 \times 10^{-16}$ \\
\hline & ${ }^{13} \mathrm{CO} v(1-0) \mathrm{P} 01$ & $9.22 \times 10^{-15}$ & $9.67 \times 10^{-16}$ \\
\hline & ${ }^{13} \mathrm{CO} v(1-0) \mathrm{P} 03$ & $1.09 \times 10^{-14}$ & $1.08 \times 10^{-15}$ \\
\hline & ${ }^{13} \mathrm{CO} v(1-0) \mathrm{P} 21$ & $2.32 \times 10^{-14}$ & $1.14 \times 10^{-15}$ \\
\hline & ${ }^{13} \mathrm{CO} v(1-0) \mathrm{P} 21$ & $1.29 \times 10^{-14}$ & $7.43 \times 10^{-16}$ \\
\hline & ${ }^{13} \mathrm{CO} v(1-0) \mathrm{P} 23$ & $1.57 \times 10^{-14}$ & $7.04 \times 10^{-16}$ \\
\hline & ${ }^{13} \mathrm{CO} v(1-0) \mathrm{P} 23$ & $1.19 \times 10^{-14}$ & $7.24 \times 10^{-16}$ \\
\hline \multirow[t]{16}{*}{ HD 190073} & ${ }^{12} \mathrm{CO} v(1-0) \mathrm{R} 08$ & $3.54 \times 10^{-14}$ & $1.79 \times 10^{-15}$ \\
\hline & ${ }^{12} \mathrm{CO} v(1-0) \mathrm{R} 06$ & $3.62 \times 10^{-14}$ & $1.58 \times 10^{-15}$ \\
\hline & ${ }^{12} \mathrm{CO} v(1-0) \mathrm{R} 06$ & $4.76 \times 10^{-14}$ & $1.80 \times 10^{-15}$ \\
\hline & ${ }^{12} \mathrm{CO} v(1-0) \mathrm{R} 04$ & $3.48 \times 10^{-14}$ & $1.62 \times 10^{-15}$ \\
\hline & ${ }^{12} \mathrm{CO} v(1-0) \mathrm{R} 04$ & $3.86 \times 10^{-14}$ & $1.59 \times 10^{-15}$ \\
\hline & ${ }^{12} \mathrm{CO} v(1-0) \mathrm{R} 02$ & $3.97 \times 10^{-14}$ & $1.87 \times 10^{-15}$ \\
\hline & ${ }^{12} \mathrm{CO} v(1-0) \mathrm{R} 01$ & $3.37 \times 10^{-14}$ & $1.31 \times 10^{-15}$ \\
\hline & ${ }^{12} \mathrm{CO} v(1-0) \mathrm{P} 02$ & $3.98 \times 10^{-14}$ & $1.43 \times 10^{-15}$ \\
\hline & ${ }^{12} \mathrm{CO} v(1-0) \mathrm{P} 02$ & $5.65 \times 10^{-14}$ & $2.54 \times 10^{-15}$ \\
\hline & ${ }^{12} \mathrm{CO} v(1-0) \mathrm{P} 05$ & $3.33 \times 10^{-14}$ & $2.88 \times 10^{-15}$ \\
\hline & ${ }^{12} \mathrm{CO} v(1-0) \mathrm{P} 06$ & $6.21 \times 10^{-14}$ & $3.40 \times 10^{-15}$ \\
\hline & ${ }^{12} \mathrm{CO} v(1-0) \mathrm{P} 08$ & $4.59 \times 10^{-14}$ & $2.88 \times 10^{-15}$ \\
\hline & ${ }^{12} \mathrm{CO} v(1-0) \mathrm{P} 08$ & $7.63 \times 10^{-14}$ & $1.21 \times 10^{-14}$ \\
\hline & ${ }^{12} \mathrm{CO} v(1-0) \mathrm{P} 09$ & $8.74 \times 10^{-14}$ & $1.24 \times 10^{-14}$ \\
\hline & ${ }^{12} \mathrm{CO} v(1-0) \mathrm{P} 30$ & $5.55 \times 10^{-14}$ & $1.83 \times 10^{-15}$ \\
\hline & ${ }^{12} \mathrm{CO} v(1-0) \mathrm{P} 32$ & $4.69 \times 10^{-14}$ & $1.62 \times 10^{-15}$ \\
\hline
\end{tabular}

Table B.4. continued.

\begin{tabular}{|c|c|c|c|}
\hline Target & Line id & $\begin{array}{l}\text { Line flux } \\
{\left[\mathrm{erg} \mathrm{cm}^{-2} \mathrm{~s}^{-1} \text { ] }\right.}\end{array}$ & $\begin{array}{l}\text { Error } \\
{\left[\mathrm{erg} \mathrm{cm}^{-2} \mathrm{~s}^{-1}\right]}\end{array}$ \\
\hline & ${ }^{12} \mathrm{CO} v(1-0) \mathrm{P} 32$ & $4.33 \times 10^{-14}$ & $1.49 \times 10^{-15}$ \\
\hline & ${ }^{12} \mathrm{CO} v(1-0) \mathrm{P} 36$ & $3.25 \times 10^{-14}$ & $1.49 \times 10^{-15}$ \\
\hline & ${ }^{12} \mathrm{CO} v(1-0) \mathrm{P} 36$ & $3.07 \times 10^{-14}$ & $1.33 \times 10^{-15}$ \\
\hline & ${ }^{12} \mathrm{CO} v(1-0) \mathrm{P} 37$ & $3.40 \times 10^{-14}$ & $1.38 \times 10^{-15}$ \\
\hline & ${ }^{12} \mathrm{CO} v(1-0) \mathrm{P} 38$ & $3.44 \times 10^{-14}$ & $3.04 \times 10^{-15}$ \\
\hline \multirow[t]{12}{*}{ HD 190073} & ${ }^{12} \mathrm{CO} v(2-1) \mathrm{R} 13$ & $2.03 \times 10^{-14}$ & $8.11 \times 10^{-16}$ \\
\hline & ${ }^{12} \mathrm{CO} v(2-1) \mathrm{R} 12$ & $2.11 \times 10^{-14}$ & $8.38 \times 10^{-16}$ \\
\hline & ${ }^{12} \mathrm{CO} v(2-1) \mathrm{R} 11$ & $2.07 \times 10^{-14}$ & $9.18 \times 10^{-16}$ \\
\hline & ${ }^{12} \mathrm{CO} v(2-1) \mathrm{R} 06$ & $2.52 \times 10^{-14}$ & $8.43 \times 10^{-16}$ \\
\hline & ${ }^{12} \mathrm{CO} v(2-1) \mathrm{R} 06$ & $1.87 \times 10^{-14}$ & $7.00 \times 10^{-16}$ \\
\hline & ${ }^{12} \mathrm{CO} v(2-1) \mathrm{P} 25$ & $1.49 \times 10^{-14}$ & $7.91 \times 10^{-16}$ \\
\hline & ${ }^{12} \mathrm{CO} v(2-1) \mathrm{P} 25$ & $1.88 \times 10^{-14}$ & $3.43 \times 10^{-15}$ \\
\hline & ${ }^{12} \mathrm{CO} v(2-1) \mathrm{P} 27$ & $1.82 \times 10^{-14}$ & $9.49 \times 10^{-16}$ \\
\hline & ${ }^{12} \mathrm{CO} v(2-1) \mathrm{P} 28$ & $1.83 \times 10^{-14}$ & $8.80 \times 10^{-16}$ \\
\hline & ${ }^{12} \mathrm{CO} v(2-1) \mathrm{P} 31$ & $1.69 \times 10^{-14}$ & $1.44 \times 10^{-15}$ \\
\hline & ${ }^{12} \mathrm{CO} v(2-1) \mathrm{P} 31$ & $1.99 \times 10^{-14}$ & $5.13 \times 10^{-15}$ \\
\hline & ${ }^{12} \mathrm{CO} v(2-1) \mathrm{P} 32$ & $1.63 \times 10^{-14}$ & $2.56 \times 10^{-15}$ \\
\hline \multirow[t]{13}{*}{ HD 98922} & ${ }^{13} \mathrm{CO} v(1-0) \mathrm{R} 16$ & $8.83 \times 10^{-15}$ & $6.84 \times 10^{-16}$ \\
\hline & ${ }^{13} \mathrm{CO} v(1-0) \mathrm{R} 15$ & $1.00 \times 10^{-14}$ & $5.15 \times 10^{-16}$ \\
\hline & ${ }^{13} \mathrm{CO} v(1-0) \mathrm{R} 12$ & $1.09 \times 10^{-14}$ & $5.60 \times 10^{-16}$ \\
\hline & ${ }^{13} \mathrm{CO} v(1-0) \mathrm{R} 10$ & $1.26 \times 10^{-14}$ & $7.33 \times 10^{-16}$ \\
\hline & ${ }^{13} \mathrm{CO} v(1-0) \mathrm{R} 05$ & $1.49 \times 10^{-14}$ & $2.56 \times 10^{-15}$ \\
\hline & ${ }^{13} \mathrm{CO} v(1-0) \mathrm{R} 03$ & $1.23 \times 10^{-14}$ & $1.42 \times 10^{-15}$ \\
\hline & ${ }^{13} \mathrm{CO} v(1-0) \mathrm{P} 01$ & $1.09 \times 10^{-14}$ & $3.01 \times 10^{-15}$ \\
\hline & ${ }^{13} \mathrm{CO} v(1-0) \mathrm{P} 21$ & $1.30 \times 10^{-14}$ & $4.52 \times 10^{-15}$ \\
\hline & ${ }^{13} \mathrm{CO} v(1-0) \mathrm{P} 21$ & $8.70 \times 10^{-15}$ & $2.46 \times 10^{-15}$ \\
\hline & ${ }^{13} \mathrm{CO} v(1-0) \mathrm{P} 25$ & $1.16 \times 10^{-14}$ & $1.98 \times 10^{-15}$ \\
\hline & ${ }^{13} \mathrm{CO} v(1-0) \mathrm{P} 25$ & $8.56 \times 10^{-15}$ & $2.51 \times 10^{-15}$ \\
\hline & ${ }^{13} \mathrm{CO} v(1-0) \mathrm{P} 26$ & $8.70 \times 10^{-15}$ & $2.69 \times 10^{-15}$ \\
\hline & ${ }^{13} \mathrm{CO} v(1-0) \mathrm{P} 27$ & $1.25 \times 10^{-14}$ & $3.69 \times 10^{-15}$ \\
\hline \multirow[t]{19}{*}{ HD 98922} & ${ }^{12} \mathrm{CO} v(1-0) \mathrm{R} 07$ & $5.96 \times 10^{-14}$ & $1.90 \times 10^{-15}$ \\
\hline & ${ }^{12} \mathrm{CO} v(1-0) \mathrm{R} 06$ & $5.84 \times 10^{-14}$ & $1.86 \times 10^{-15}$ \\
\hline & ${ }^{12} \mathrm{CO} v(1-0) \mathrm{R} 03$ & $4.52 \times 10^{-14}$ & $1.92 \times 10^{-15}$ \\
\hline & ${ }^{12} \mathrm{CO} v(1-0) \mathrm{R} 02$ & $4.80 \times 10^{-14}$ & $1.83 \times 10^{-15}$ \\
\hline & ${ }^{12} \mathrm{CO} v(1-0) \mathrm{R} 01$ & $4.73 \times 10^{-14}$ & $2.92 \times 10^{-15}$ \\
\hline & ${ }^{12} \mathrm{CO} v(1-0) \mathrm{P} 02$ & $3.04 \times 10^{-14}$ & $1.03 \times 10^{-15}$ \\
\hline & ${ }^{12} \mathrm{CO} v(1-0) \mathrm{P} 05$ & $3.87 \times 10^{-14}$ & $1.33 \times 10^{-15}$ \\
\hline & ${ }^{12} \mathrm{CO} v(1-0) \mathrm{P} 05$ & $3.73 \times 10^{-14}$ & $1.59 \times 10^{-15}$ \\
\hline & ${ }^{12} \mathrm{CO} v(1-0) \mathrm{P} 06$ & $4.43 \times 10^{-14}$ & $3.04 \times 10^{-15}$ \\
\hline & ${ }^{12} \mathrm{CO} v(1-0) \mathrm{P} 08$ & $4.88 \times 10^{-14}$ & $1.49 \times 10^{-15}$ \\
\hline & ${ }^{12} \mathrm{CO} v(1-0) \mathrm{P} 08$ & $4.20 \times 10^{-14}$ & $1.40 \times 10^{-15}$ \\
\hline & ${ }^{12} \mathrm{CO} v(1-0) \mathrm{P} 12$ & $6.15 \times 10^{-14}$ & $3.50 \times 10^{-15}$ \\
\hline & ${ }^{12} \mathrm{CO} v(1-0) \mathrm{P} 30$ & $4.16 \times 10^{-14}$ & $1.95 \times 10^{-15}$ \\
\hline & ${ }^{12} \mathrm{CO} v(1-0) \mathrm{P} 31$ & $3.18 \times 10^{-14}$ & $5.42 \times 10^{-15}$ \\
\hline & ${ }^{12} \mathrm{CO} v(1-0) \mathrm{P} 34$ & $3.54 \times 10^{-14}$ & $8.92 \times 10^{-15}$ \\
\hline & ${ }^{12} \mathrm{CO} v(1-0) \mathrm{P} 36$ & $2.16 \times 10^{-14}$ & $9.44 \times 10^{-16}$ \\
\hline & ${ }^{12} \mathrm{CO} v(1-0) \mathrm{P} 36$ & $3.00 \times 10^{-14}$ & $1.28 \times 10^{-15}$ \\
\hline & ${ }^{12} \mathrm{CO} v(1-0) \mathrm{P} 37$ & $2.06 \times 10^{-14}$ & $9.63 \times 10^{-16}$ \\
\hline & ${ }^{12} \mathrm{CO} v(1-0) \mathrm{P} 38$ & $2.33 \times 10^{-14}$ & $1.12 \times 10^{-15}$ \\
\hline \multirow[t]{6}{*}{ HD 98922} & ${ }^{12} \mathrm{CO} v(2-1) \mathrm{R} 13$ & $1.17 \times 10^{-14}$ & $1.96 \times 10^{-15}$ \\
\hline & ${ }^{12} \mathrm{CO} v(2-1) \mathrm{R} 06$ & $2.25 \times 10^{-14}$ & $10.00 \times 10^{-15}$ \\
\hline & ${ }^{12} \mathrm{CO} v(2-1) \mathrm{P} 25$ & $1.37 \times 10^{-14}$ & $2.10 \times 10^{-15}$ \\
\hline & ${ }^{12} \mathrm{CO} v(2-1) \mathrm{P} 27$ & $1.04 \times 10^{-14}$ & $1.60 \times 10^{-15}$ \\
\hline & ${ }^{12} \mathrm{CO} v(2-1) \mathrm{P} 28$ & $1.33 \times 10^{-14}$ & $4.74 \times 10^{-15}$ \\
\hline & ${ }^{12} \mathrm{CO} v(2-1) \mathrm{P} 28$ & $1.38 \times 10^{-14}$ & $4.92 \times 10^{-15}$ \\
\hline \multirow[t]{6}{*}{ HD 95881} & ${ }^{12} \mathrm{CO} v(1-0) \mathrm{R} 06$ & $5.07 \times 10^{-14}$ & $4.01 \times 10^{-15}$ \\
\hline & ${ }^{12} \mathrm{CO} v(1-0) \mathrm{R} 05$ & $5.48 \times 10^{-14}$ & $2.99 \times 10^{-15}$ \\
\hline & ${ }^{12} \mathrm{CO} v(1-0) \mathrm{P} 08$ & $5.65 \times 10^{-14}$ & $2.17 \times 10^{-15}$ \\
\hline & ${ }^{12} \mathrm{CO} v(1-0) \mathrm{P} 11$ & $4.16 \times 10^{-14}$ & $3.30 \times 10^{-15}$ \\
\hline & ${ }^{12} \mathrm{CO} v(1-0) \mathrm{P} 32$ & $4.07 \times 10^{-14}$ & $1.84 \times 10^{-15}$ \\
\hline & ${ }^{12} \mathrm{CO} v(1-0) \mathrm{P} 36$ & $1.74 \times 10^{-14}$ & $1.70 \times 10^{-15}$ \\
\hline
\end{tabular}


Table B.4. continued.

\begin{tabular}{|c|c|c|c|}
\hline Target & Line id & $\begin{array}{l}\text { Line flux } \\
\text { [erg cm } \mathrm{cm}^{-2} \mathrm{~s}^{-1} \text { ] }\end{array}$ & $\begin{array}{l}\text { Error } \\
{\left[\mathrm{erg} \mathrm{cm}^{-2} \mathrm{~s}^{-1} \text { ] }\right.}\end{array}$ \\
\hline & ${ }^{12} \mathrm{CO} v(1-0) \mathrm{P} 37$ & $3.31 \times 10^{-14}$ & $2.12 \times 10^{-15}$ \\
\hline \multirow[t]{8}{*}{ HD 95881} & ${ }^{13} \mathrm{CO} v(1-0) \mathrm{R} 16$ & $8.56 \times 10^{-15}$ & $1.61 \times 10^{-15}$ \\
\hline & ${ }^{13} \mathrm{CO} v(1-0) \mathrm{R} 13$ & $1.00 \times 10^{-14}$ & $6.15 \times 10^{-16}$ \\
\hline & ${ }^{13} \mathrm{CO} v(1-0) \mathrm{R} 10$ & $1.38 \times 10^{-14}$ & $2.12 \times 10^{-15}$ \\
\hline & ${ }^{13} \mathrm{CO} v(1-0) \mathrm{R} 10$ & $9.88 \times 10^{-15}$ & $1.81 \times 10^{-15}$ \\
\hline & ${ }^{13} \mathrm{CO} v(1-0) \mathrm{R} 09$ & $1.40 \times 10^{-14}$ & $2.11 \times 10^{-15}$ \\
\hline & ${ }^{13} \mathrm{CO} v(1-0) \mathrm{R} 04$ & $1.05 \times 10^{-14}$ & $1.89 \times 10^{-15}$ \\
\hline & ${ }^{13} \mathrm{CO} v(1-0) \mathrm{R} 03$ & $1.12 \times 10^{-14}$ & $2.13 \times 10^{-15}$ \\
\hline & ${ }^{13} \mathrm{CO} v(1-0) \mathrm{P} 25$ & $5.27 \times 10^{-15}$ & $2.74 \times 10^{-15}$ \\
\hline \multirow[t]{21}{*}{$\mathrm{R} \mathrm{CrA}$} & ${ }^{12} \mathrm{CO} v(1-0) \mathrm{P} 04$ & $4.05 \times 10^{-14}$ & $5.65 \times 10^{-15}$ \\
\hline & ${ }^{12} \mathrm{CO} v(1-0) \mathrm{P} 05$ & $4.11 \times 10^{-14}$ & $5.95 \times 10^{-15}$ \\
\hline & ${ }^{12} \mathrm{CO} v(1-0) \mathrm{P} 05$ & $7.02 \times 10^{-14}$ & $3.33 \times 10^{-14}$ \\
\hline & ${ }^{12} \mathrm{CO} v(1-0) \mathrm{P} 06$ & $4.37 \times 10^{-14}$ & $1.73 \times 10^{-14}$ \\
\hline & ${ }^{12} \mathrm{CO} v(1-0) \mathrm{P} 07$ & $2.71 \times 10^{-14}$ & $3.21 \times 10^{-15}$ \\
\hline & ${ }^{12} \mathrm{CO} v(1-0) \mathrm{P} 07$ & $4.52 \times 10^{-14}$ & $1.34 \times 10^{-14}$ \\
\hline & ${ }^{12} \mathrm{CO} v(1-0) \mathrm{P} 08$ & $3.93 \times 10^{-14}$ & $6.33 \times 10^{-15}$ \\
\hline & ${ }^{12} \mathrm{CO} v(1-0) \mathrm{P} 08$ & $3.95 \times 10^{-14}$ & $1.62 \times 10^{-14}$ \\
\hline & ${ }^{12} \mathrm{CO} v(1-0) \mathrm{P} 11$ & $4.77 \times 10^{-14}$ & $4.96 \times 10^{-15}$ \\
\hline & ${ }^{12} \mathrm{CO} v(1-0) \mathrm{P} 11$ & $4.10 \times 10^{-14}$ & $2.34 \times 10^{-14}$ \\
\hline & ${ }^{12} \mathrm{CO} v(1-0) \mathrm{P} 12$ & $4.10 \times 10^{-14}$ & $1.49 \times 10^{-14}$ \\
\hline & ${ }^{12} \mathrm{CO} v(1-0) \mathrm{P} 13$ & $4.51 \times 10^{-14}$ & $7.01 \times 10^{-15}$ \\
\hline & ${ }^{12} \mathrm{CO} v(1-0) \mathrm{P} 14$ & $4.61 \times 10^{-14}$ & $1.67 \times 10^{-14}$ \\
\hline & ${ }^{12} \mathrm{CO} v(1-0) \mathrm{P} 30$ & $3.16 \times 10^{-14}$ & $1.22 \times 10^{-14}$ \\
\hline & ${ }^{12} \mathrm{CO} v(1-0) \mathrm{P} 31$ & $2.60 \times 10^{-14}$ & $7.24 \times 10^{-15}$ \\
\hline & ${ }^{12} \mathrm{CO} v(1-0) \mathrm{P} 32$ & $1.98 \times 10^{-14}$ & $9.90 \times 10^{-15}$ \\
\hline & ${ }^{12} \mathrm{CO} v(1-0) \mathrm{P} 32$ & $1.95 \times 10^{-14}$ & $4.27 \times 10^{-15}$ \\
\hline & ${ }^{12} \mathrm{CO} v(1-0) \mathrm{P} 34$ & $1.66 \times 10^{-14}$ & $4.13 \times 10^{-15}$ \\
\hline & ${ }^{12} \mathrm{CO} v(1-0) \mathrm{P} 36$ & $1.67 \times 10^{-14}$ & $5.25 \times 10^{-15}$ \\
\hline & ${ }^{12} \mathrm{CO} v(1-0) \mathrm{P} 37$ & $2.07 \times 10^{-14}$ & $4.88 \times 10^{-15}$ \\
\hline & ${ }^{12} \mathrm{CO} v(1-0) \mathrm{P} 37$ & $2.11 \times 10^{-14}$ & $5.39 \times 10^{-15}$ \\
\hline \multirow[t]{25}{*}{ HD 135344B } & ${ }^{12} \mathrm{CO} v(1-0) \mathrm{R} 08$ & $6.77 \times 10^{-14}$ & $1.08 \times 10^{-14}$ \\
\hline & ${ }^{12} \mathrm{CO} v(1-0) \mathrm{R} 07$ & $5.63 \times 10^{-14}$ & $3.47 \times 10^{-15}$ \\
\hline & ${ }^{12} \mathrm{CO} v(1-0) \mathrm{R} 07$ & $5.24 \times 10^{-14}$ & $1.79 \times 10^{-15}$ \\
\hline & ${ }^{12} \mathrm{CO} v(1-0) \mathrm{R} 06$ & $4.56 \times 10^{-14}$ & $3.82 \times 10^{-15}$ \\
\hline & ${ }^{12} \mathrm{CO} v(1-0) \mathrm{R} 06$ & $5.01 \times 10^{-14}$ & $1.86 \times 10^{-15}$ \\
\hline & ${ }^{12} \mathrm{CO} v(1-0) \mathrm{R} 05$ & $4.85 \times 10^{-14}$ & $2.36 \times 10^{-15}$ \\
\hline & ${ }^{12} \mathrm{CO} v(1-0) \mathrm{R} 05$ & $4.64 \times 10^{-14}$ & $1.68 \times 10^{-15}$ \\
\hline & ${ }^{12} \mathrm{CO} v(1-0) \mathrm{R} 03$ & $3.35 \times 10^{-14}$ & $2.46 \times 10^{-15}$ \\
\hline & ${ }^{12} \mathrm{CO} v(1-0) \mathrm{R} 02$ & $4.14 \times 10^{-14}$ & $2.06 \times 10^{-15}$ \\
\hline & ${ }^{12} \mathrm{CO} v(1-0) \mathrm{R} 02$ & $4.16 \times 10^{-14}$ & $1.40 \times 10^{-15}$ \\
\hline & ${ }^{12} \mathrm{CO} v(1-0) \mathrm{R} 01$ & $3.41 \times 10^{-14}$ & $2.22 \times 10^{-15}$ \\
\hline & ${ }^{12} \mathrm{CO} v(1-0) \mathrm{R} 01$ & $3.28 \times 10^{-14}$ & $1.27 \times 10^{-15}$ \\
\hline & ${ }^{12} \mathrm{CO} v(1-0) \mathrm{P} 02$ & $2.98 \times 10^{-14}$ & $2.29 \times 10^{-15}$ \\
\hline & ${ }^{12} \mathrm{CO} v(1-0) \mathrm{P} 02$ & $2.98 \times 10^{-14}$ & $1.12 \times 10^{-15}$ \\
\hline & ${ }^{12} \mathrm{CO} v(1-0) \mathrm{P} 04$ & $6.98 \times 10^{-14}$ & $4.23 \times 10^{-15}$ \\
\hline & ${ }^{12} \mathrm{CO} v(1-0) \mathrm{P} 06$ & $5.77 \times 10^{-14}$ & $2.71 \times 10^{-14}$ \\
\hline & ${ }^{12} \mathrm{CO} v(1-0) \mathrm{P} 10$ & $4.39 \times 10^{-14}$ & $1.25 \times 10^{-14}$ \\
\hline & ${ }^{12} \mathrm{CO} v(1-0) \mathrm{P} 10$ & $7.66 \times 10^{-14}$ & $4.43 \times 10^{-15}$ \\
\hline & ${ }^{12} \mathrm{CO} v(1-0) \mathrm{P} 11$ & $5.05 \times 10^{-14}$ & $8.29 \times 10^{-15}$ \\
\hline & ${ }^{12} \mathrm{CO} v(1-0) \mathrm{P} 30$ & $1.84 \times 10^{-14}$ & $3.00 \times 10^{-15}$ \\
\hline & ${ }^{12} \mathrm{CO} v(1-0) \mathrm{P} 31$ & $1.81 \times 10^{-14}$ & $3.13 \times 10^{-15}$ \\
\hline & ${ }^{12} \mathrm{CO} v(1-0) \mathrm{P} 32$ & $2.53 \times 10^{-14}$ & $8.65 \times 10^{-15}$ \\
\hline & ${ }^{12} \mathrm{CO} v(1-0) \mathrm{P} 32$ & $1.23 \times 10^{-14}$ & $5.11 \times 10^{-15}$ \\
\hline & ${ }^{12} \mathrm{CO} v(1-0) \mathrm{P} 36$ & $1.69 \times 10^{-14}$ & $6.42 \times 10^{-15}$ \\
\hline & ${ }^{12} \mathrm{CO} v(1-0) \mathrm{P} 38$ & $1.38 \times 10^{-14}$ & $5.31 \times 10^{-15}$ \\
\hline \multirow[t]{4}{*}{ HD 150193} & ${ }^{12} \mathrm{CO} v(1-0) \mathrm{R} 06$ & $6.83 \times 10^{-14}$ & $2.58 \times 10^{-14}$ \\
\hline & ${ }^{12} \mathrm{CO} v(1-0) \mathrm{P} 08$ & $8.22 \times 10^{-14}$ & $1.94 \times 10^{-14}$ \\
\hline & ${ }^{12} \mathrm{CO} v(1-0) \mathrm{P} 30$ & $9.61 \times 10^{-14}$ & $4.48 \times 10^{-15}$ \\
\hline & ${ }^{12} \mathrm{CO} v(1-0) \mathrm{P} 32$ & $6.50 \times 10^{-14}$ & $2.52 \times 10^{-15}$ \\
\hline
\end{tabular}

Table B.4. continued.

\begin{tabular}{llll}
\hline \hline Target & Line id & $\begin{array}{l}\text { Line flux } \\
{\left[\mathrm{erg} \mathrm{cm}^{-2} \mathrm{~s}^{-1}\right]}\end{array}$ & $\begin{array}{l}\text { Error } \\
{\left[\mathrm{erg} \mathrm{cm}^{-2} \mathrm{~s}^{-1}\right]}\end{array}$ \\
\hline & ${ }^{12} \mathrm{CO} v(1-0) \mathrm{P} 32$ & $7.06 \times 10^{-14}$ & $3.95 \times 10^{-15}$ \\
& ${ }^{12} \mathrm{CO} v(1-0) \mathrm{P} 36$ & $5.57 \times 10^{-14}$ & $4.94 \times 10^{-15}$ \\
\hline HD 104237 & ${ }^{12} \mathrm{CO} v(1-0) \mathrm{R} 08$ & $8.21 \times 10^{-14}$ & $1.52 \times 10^{-14}$ \\
& ${ }^{12} \mathrm{CO} v(1-0) \mathrm{R} 07$ & $7.76 \times 10^{-14}$ & $9.49 \times 10^{-15}$ \\
& ${ }^{12} \mathrm{CO} v(1-0) \mathrm{R} 06$ & $5.38 \times 10^{-14}$ & $9.10 \times 10^{-15}$ \\
& ${ }^{12} \mathrm{CO} v(1-0) \mathrm{R} 05$ & $4.89 \times 10^{-14}$ & $3.08 \times 10^{-14}$ \\
& ${ }^{12} \mathrm{CO} v(1-0) \mathrm{P} 06$ & $5.71 \times 10^{-14}$ & $8.84 \times 10^{-15}$ \\
& ${ }^{12} \mathrm{CO} v(1-0) \mathrm{P} 08$ & $8.47 \times 10^{-14}$ & $1.48 \times 10^{-14}$ \\
& ${ }^{12} \mathrm{CO} v(1-0) \mathrm{P} 10$ & $7.60 \times 10^{-14}$ & $1.88 \times 10^{-14}$ \\
\hline
\end{tabular}

